\title{
SWEEP STABILITY CHARACTERIZATION OF A VERNIER-TUNED DISTRIBUTED BRAGG REFLECTOR (VT-DBR) ALL- SEMICONDUCTOR TUNABLE SWEPT LASER SYSTEM AT 1550 NM FOR SENSING APPLICATIONS
}

\author{
A Thesis \\ presented to \\ the Faculty of California Polytechnic State University, \\ San Luis Obispo
}

\author{
In Partial Fulfillment \\ of the Requirements for the Degree \\ Master of Science in Electrical Engineering
}

by

Roric Christian Martens Biersach

June 2015 
(C) 2015

Roric Christian Martens Biersach

ALL RIGHTS RESERVED 


\section{COMMITTEE MEMBERSHIP}

TITLE:

Sweep Stability Characterization of a Vernier-Tuned Distributed Bragg Reflector (VT-DBR) All-

Semiconductor Tunable Swept Laser System at $1550 \mathrm{~nm}$ for Sensing Applications

AUTHOR:

Roric Christian Martens Biersach

DATE SUBMITTED:

June 2015

COMMITTEE CHAIR: $\quad$ Dennis Derickson, Ph.D.

Professor of Electrical Engineering

COMMITTEE MEMBER: $\quad$ Samuel Agbo, Ph.D.

Professor of Electrical Engineering

COMMITTEE MEMBER: Xiaomin Jin, Ph.D.

Associate Professor of Electrical Engineering

COMMITTEE MEMBER: Jason Ensher, Ph.D.

Vice President of Engineering at Insight Photonic

Solutions, Inc. 


\begin{abstract}
Sweep Stability Characterization of a Vernier-Tuned Distributed

Bragg Reflector (VT-DBR) All-Semiconductor Tunable Swept

Laser System at $1550 \mathrm{~nm}$ for Sensing Applications
\end{abstract}

\title{
Roric Christian Martens Biersach
}

The short-term jitter and longer-term wander of the frequency sweep profile of a Vernier-Tuned Distributed Bragg Reflector (VT-DBR) laser at $1550 \mathrm{~nm}$ used in optical coherence tomography (OCT) and other sensing applications is characterized in this work. The VT-DBR has demonstrated success in source-swept OCT (SSOCT), performing both intensity and phase-sensitive OCT.

The purpose of this paper is to investigate one of the unique aspects of the VTDBR laser that makes it successful in OCT: the stability of the linear optical frequency sweep. A highly stable linear optical frequency sweep implies benefits for further fiber sensing applications including fiber Bragg grating and spectroscopy sensors.

Short-term jitter measurements of the optical frequency sweep are taken using a 3-cavity $100 \mathrm{GHz}$ free spectral range solid etalon, an athermal fiber Bragg grating, a molecular-based gas absorption reference cell, and a Mach-Zehnder interferometer. These four optical filters are used to convert time fluctuations into intensity fluctuations that can be measured by high speed optical receivers. Short term jitter values on the order of $0.5-0.6 \mathrm{pm}$ RMS (63-82 MHz RMS) are typical values in the VT-DBR lasers systems characterized in this work. This level of jitter is compelling for OCT and fibersensing applications.

Longer-term wander is characterized using a multiple-gas absorption reference cell. The long term stability and temperature insensitivity of the absorption cell is ideal for long-term wander characterization of the laser frequency sweeps. Wander values on the order of $2.6 \mathrm{pm}$ of wavelength shift over an 8 hour time frame are reported in this work. The slope of the frequency versus time function of the laser sweep, on the order of $100 \mathrm{MHz} / \mathrm{sample}$, is found to deviate by no more than $0.03 \%$ over the same 8 hour time frame. Both the long term wavelength shift and consistency of the slope indicate that these sources will perform well in OCT and fiber sensing applications.

Mechanisms responsible for short-term jitter and longer-term wavelength wander likely include contributions from the laser source itself and from the high speed electronic drive circuitry that creates the wavelength ramp. Investigation of ambient temperature's influence on the wavelength wander is also highlighted in the work.

Keywords: Vernier-Tuned Distributed Bragg Reflector, VT-DBR, Swept Laser, Akinetic, OCT, SSOCT, PhOCT, 1550 nm, Jitter, Wander 


\section{ACKNOWLEDGMENTS}

This project would not be possible without the unwavering support, encouragement, and advice from Drs. Dennis Derickson and Jason Ensher. Without their correspondences, long phone conversations, patience, and insight, this project would not be possible. Insight Photonic Solutions provided funding and hardware used for this project for which I am grateful.

I would like to thank Dr. Christian Karnutsch (Hochschule Karlsruhe, Technik und Wirtschaft) for inspiring me to pursue the field of optics and photonics.

For the endless technical help and support at any moment of the day, I extend a thank you to Jaime Carmo and Corey Ricketts of the Electrical Engineering department.

I would like to thank Drs. Xiaomin Jin and Samuel Agbo for supporting my work and participating on my thesis committee.

Last, I would like to thank my sister, Katryna, for inspiring me to undertake this endeavor. I express gratitude for the support, love, and encouragement from my mother, father, and Klaus Mazura throughout this project. 


\section{TABLE OF CONTENTS}

Page

LIST OF TABLES …...................................................................................... vii

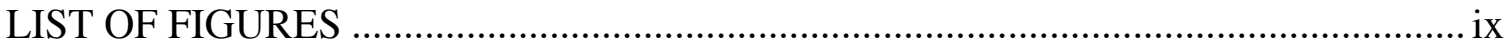

CHAPTER

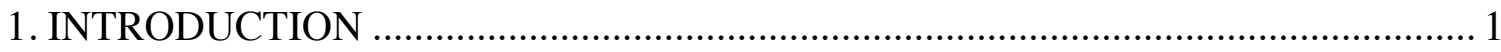

1.1 Biomedical Imaging: Optical Coherence Tomography (OCT) ............................ 2

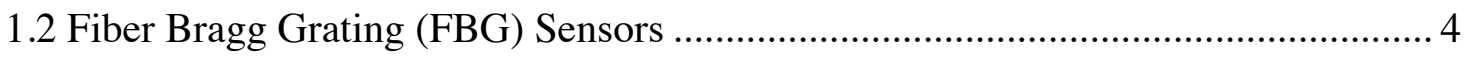

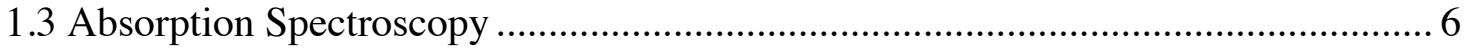

1.4 Purpose of Study ......................................................................................... 7

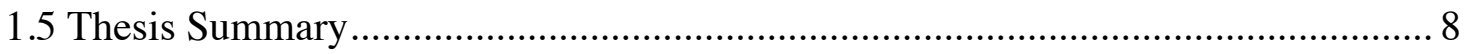

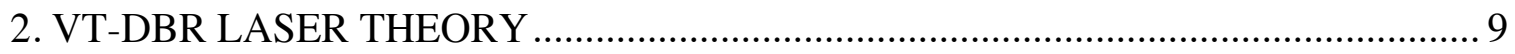

2.1 Laser Physics Background ..................................................................... 9

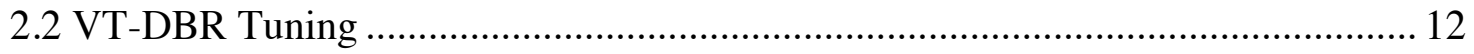

2.3 VT-DBR Laser System Performance Advantages .......................................... 17

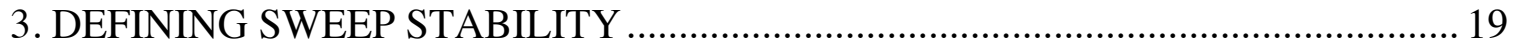

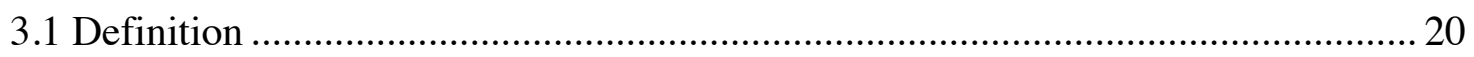

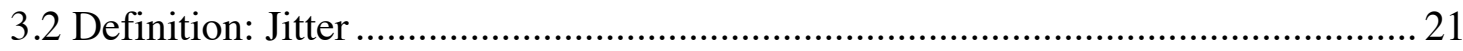

3.3 Definition: Wander .................................................................................... 22

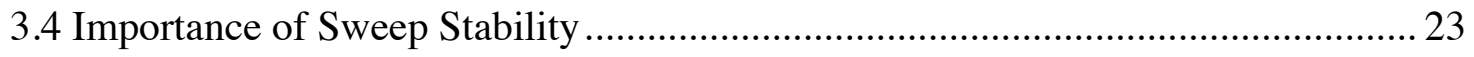

3.5 Assumptions and Adaptations Used in this Study .......................................... 24

4. JITTER CHARACTERIZATION .................................................................... 25

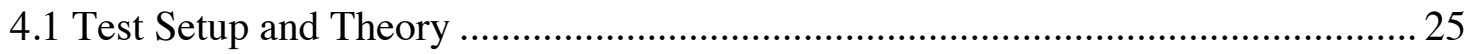

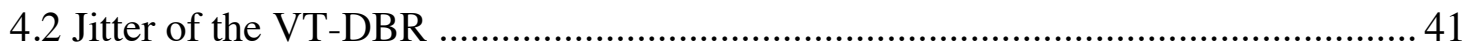


4.3 Jitter of a 2nd Generation VT-DBR ............................................................. 45

4.4 Summary and Analysis of Jitter Results .................................................... 47

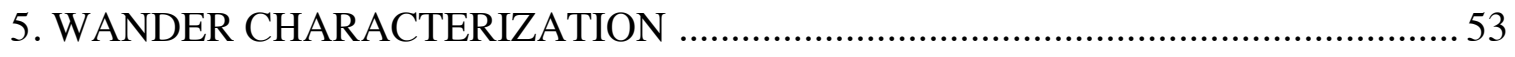

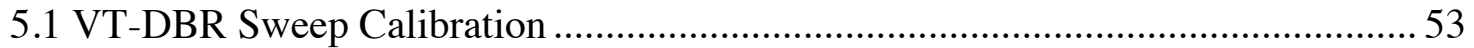

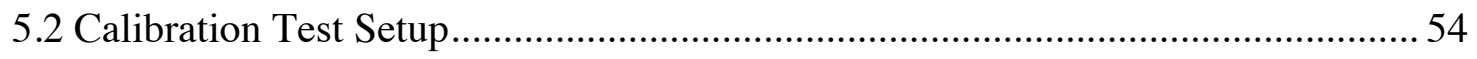

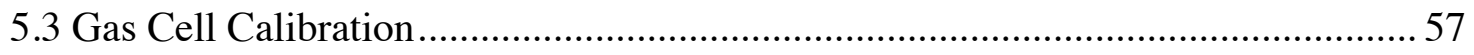

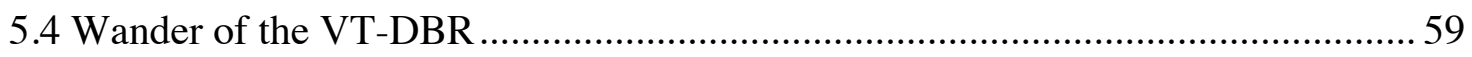

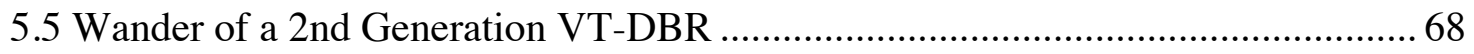

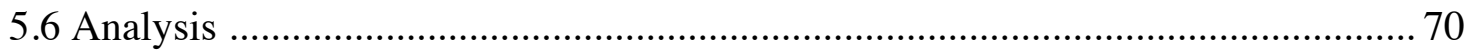

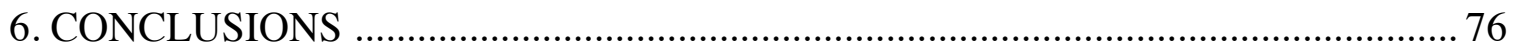

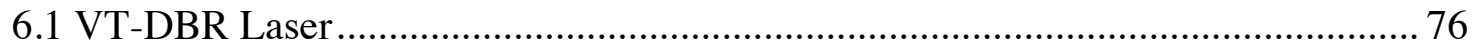

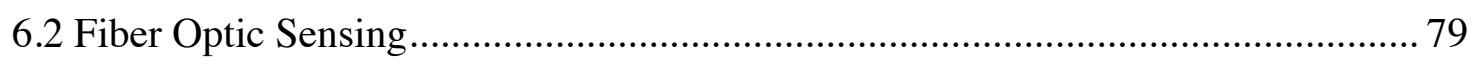

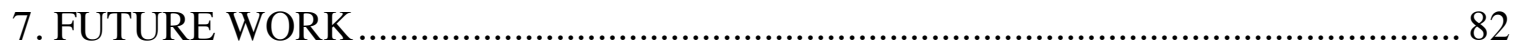

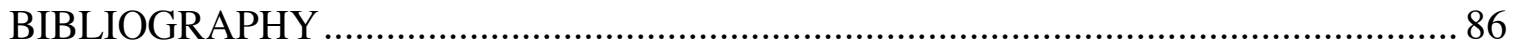




\section{LIST OF TABLES}

Table

Page

1 - Typical VT-DBR laser parameters for jitter characterization ................................29

2 - Gas cells used in this project. The WA-1530-1560 is used for jitter characterization due to its high number of absorption features in the range of a VT-DBR sweep..............31

3 - Summary of jitter measurements conducted using an athermal fiber Bragg grating. The rising and falling edge of the reflected Bragg wavelength are monitored and recorded. Results verify findings from previous measurements using other DUTs

4 - Summary of jitter in picometers from the different methods of interrogation undertaken in this project for the first generation VT-DBR module. For each of these results, observation times of no more than 5 seconds were allowed.

5 - Parameters that are adjustable in the developed data acquisition system using NI

LabView to make data collection automated and feasible.

6 - Gas cells used in this project. The WA-900-1638 is used to characterize wander due to its lower number of absorption features in the range of the VT-DBR sweep.........60

7 - Summary of jitter measurements conducted using an athermal fiber Bragg grating. The rising and falling edge of the reflected Bragg wavelength are monitored and recorded. Results verify findings from previous measurements using other DUTs 


\section{LIST OF FIGURES}

Figure

Page

1-1 - A comparison of established biomedical imaging techniques displaying the unique market niche of OCT [8]

1-2 - A typical SSOCT test setup. There is a sampling output (to the photo detector)

and two sample arms, one as a reference with a fixed mirror [8]

1-3 - Fiber Bragg grating (FBG) sensors reflect a characteristic Bragg wavelength, defined by the periodic refractive index changes in the fiber [47]....

1-4 - Varying temperature, pressure, or strain on an FBG causes the Bragg wavelength to shift due to a shift in the grating period [49]......

1-5 - An example absorption spectrum of Copper Sullphate in water. The lower wavelengths are not absorbed as compared to the higher wavelengths [48].

1-6 - A laser sweep begins with an initial optical frequency at initial time (to) and sweeps from fmax to fmin at a rate equal to the optical frequency step, $\Delta \mathrm{F}$.

2-1 - A laser is composed of 3 fundamental components: an optical or electrical energy pump, an optical resonator composed of a fully reflecting mirror and a partially reflecting mirror, and a gain medium where stimulated emission takes place......9

2-2 - Spontaneous emission is the light-producing mechanism in most light sources. An atom in an excited state transitions to a lower state spontaneously emitting a photon in the process

2-3 - In stimulated emission, a photon causes an excited atom to transition to its

ground state, emitting two phase-locked photons of identical wavelength.....

2-4 - Energy level diagram of a 3-level laser showing a state of population inversion where more electrons are excited than in the ground state.

2-5 - Bragg gratings are used to create distributed feedback in a DBR grating. These gratings are composed of materials of varying refractive index

2-6 - The VT-DBR has a sampled grating distributed Bragg reflector structure controlled by 5 separate currents for wavelength tuning [6]

2-7 - The Vernier effect is most often used for precise distance or angle measurements, but is exploited by the VT-DBR in a unique fashion [1]...... 
2-8 - The VT-DBR is contained on a single monolithic chip with 5 control currents to conrol the front mirror, back mirror, phase, gain, and semiconductor optical amplifier [2].

2-9 - The VT-DBR Akinetic laser system contains complex drive circuitry and algorithms as well as a Thermo-Electric Cooler, all of which contribute noise to the system. The TEC attempts to regulate the temperature of the laser chip, but induces some amount of thermal noise into the system. The digital-to-analog converters (D/A) and trans-impedance amplifiers (VI) have a shot noise limit which limits the noise induced into the laser segments and, thus, their output wavelength selectivity and power. This noise ultimately contributes to the short- and long-term stability of the optical sweep [50].

2-10 - It is advantageous in some applications to quickly sweep across two, nonconjoined sweep ranges. The VT-DBR has the ability to output such a noncontiguous laser sweep rapidly, which is impossible with other swept lasers [4]. For example, a sweep of $50 \mathrm{~nm}$ at a center wavelength of $1310 \mathrm{~nm}$ may take place followed rapidly by another $50 \mathrm{~nm}$ sweep at a $1550 \mathrm{~nm}$ center wavelength.

2-11 - The VT-DBR system allows for a high degree of sweep programmability, as illustrated by the blue arrows. A user may customize parameters such as number of measurement points, sweep width, coherence length, sweep speed, duty cycle, power profile, and others [4]

3-1 - A typical sweep of the VT-DBR is linear in either optical frequency or wavelength. Either is an appropriate description for the sweep of the laser

3-2 - An ideal laser sweep is described by its minimum wavelength, $\lambda 0$ (offset) and optical frequency step (slope), $\Delta \mathrm{F}$. Stability of the optical frequency sweep is defined through deviations in linearity, Ferror, and minimum sweep wavelength, $\Delta \lambda 0$.

3-3 - Traditional jitter measurements are made on timing signals or clocks. These signals are well-defined and periodic in nature [10].

4-1 - The test setup used to measure jitter and wander in the VT-DBR optical frequency sweep. The laser system is controlled via Ethernet. The optical pathway contains an optical attenuator so that the photo detector does not saturate; variable lengths of fiber; a wavelength reference device under test (DUT); an amplified photo detector; and a low pass filter (LPF) to remove noise above $15 \mathrm{MHz}$. One reference device under test is a Hydrogen-Cyanide $(\mathrm{HCN})$ molecular-based gas absorption cell provides a wavelength reference that is highly stable over long periods of time and external environmental changes. A second reference device under test is a 3-cavity $100 \mathrm{GHz}$ free spectral range (FSR) solid etalon exhibits excellent short-term stability and contains over 100 wavelength markers throughout the laser sweep. An athermal fiber Bragg grating (FBG) and a second quad-gas reference cell are also tested to measure stability. 
4-2 - Front panel of the Laser Control software from Insight Photonic Solutions. This software interface makes it simple to modify a laser sweep in many different ways atany time. The yellow section on the left side of the interface is where a user enterssweep parameters and initiates calibrations and sweeps. The blue area of the user interface displays relevent laser performance metrics.

4-3 - The main components in the test setup shown in figure 4-1. The laser control software (figure 4-2) controls the laser system (A) through a computer and Ethernet connection. The laser output is coupled with a fiber attenuator (B) before going through reference device $2(\mathrm{C})$, a gas reference cell. The output of the reference cell is coupled onto the amplified photo detector (D) who's RF output is coupled into a low pass filter (E).

4-4 - Output signal of amplified photo detector with (right) and without (left) lowpass filter. The periodic waveforms on top represent periods of the etalon while the two captures on bottom represent histograms of two edge transitions. The peak-topeak deviation of the unfiltered histogram (bottom left) is approximately $100 \mathrm{~ns}$ while the filtered histogram (bottom right) is only about $8 \mathrm{~ns}$

4-5 - Signal-to-noise ratio (SNR) ration improvement using a $15 \mathrm{MHz}$ low pass filter after the ThorLabs photo detector. The phase-leading (pink) signal is the raw output from the amplified photo detector. This photo detector amplifies any optical input signal and noise up to $350 \mathrm{MHz}$ while the signal of interest contains features in a measurement bandwidth of no more than $10 \mathrm{MHz}$. A low-pass filter is applied to the noisy signal to obtain the phase-lagging (yellow) signal, delayed by approximately 55 ns due to the filter group delay..

4-6 - Device under test 1 is a gas reference cell. The Hydrogen Cyanide cell is used in characterizing jitter.

4-7 - Transmission spectra of WA-1530-1560 Hydrogen Cyanide cell (left) and WA-900-1638 Quad cell (right) and the range of a VT-DBR sweep is mapped on the horizontal axis in red [15].

4-8 - Threshold location on a gas cell absorption line is at the steepest rate of change, generally near full width half maximum (FWHM). A marker is placed on the vertical location at which the falling edge of this etalon pass-to-stop band transitiont is measured

4-9 - Measuring peak-to-peak jitter is a matter of finding a well-defined slope on the output of the laser. The peak-to-peak time deviation of this point on the absorption spectrum is used to compute optical frequency and wavelength jitter.

4-10 - Two histograms showing the Gaussian nature of jitter measured in all methods...35 
4-11 - The histogram created by the oscilloscope is nearly Gaussian. The standard deviation, peak-to-peak value, as well as number of tabulated results are reported

4-12 - DUT 2 is a 3-Cavity $100 \mathrm{GHz}$ Etalon with the transmission characteristic shown.

4-13 - Oscilloscope capture showing the etalon output with the VT-DBR sweeping as an input. Note that peaks of the etalon waveform are separated by $100 \mathrm{GHz}$. The data valid vector (DVV) and start sweep (SS) triggers are also shown.....

4-14 - Two histograms of a rising etalon edge (top) and two histograms of a falling etalon edge (botton. The etalon signal is shown in yellow and the histogram appears in turquoise. Note the Gaussian nature of the histograms. Each histogram has $1000+$ bins taken over 10 seconds.

4-15 - DUT 3 is a fiber Bragg grating which is temperature-compensated (athermal) and encapsulated for protection from the environment. This FBG has a maximum attenuation of $14.75 \mathrm{~dB}$ and FWHM of $0.4 \mathrm{~nm}$

4-16 - Full spectrum of gas cell (left, blue) and a zoomed in view of a single absorption peak. The blue and red curves on the right show two absorption peaks superimposed with their polynomial fit curves in red....

4-17 - Optical wavelength jitter of the VT-DBR as measured through the Hydrogen Cyanide gas cell with 51 absorption features across nearly the entire sweep range of the laser. The laser is swept at $4 \mathrm{~mW}$ and an $8.2 \mathrm{kHz}$ sweep repetition rate.

4-18 - RMS wavelength jitter measured using the etalon. Jitter values are aligned with their approximate wavelengths, calculated based on the $100 \mathrm{GHz}$ free spectral range of the etalon.

4-19 - RMS Wavelength jitter measured using the solid etalon DUT and $25 \mathrm{~km}$ of fiber in the optical signal pathway. Results are on the same order of magnitude as other measurements with very little fiber length

4-20 - The new 2nd generation VT-DBR laser system with improved drive circuitry and noise reduction mechanisms exhibits jitter on the same order as the previous generation, but slightly lower with less jitter "spikes" or outliers. The ramp nature of the jitter vs. wavelength plot is noted.

4-21 - A histogram of a sharp transition measuring intrinsic jitter of the VT-DBR system. Note the Gaussian nature of the distribution and the peak-to-peak value of $5.455 \mathrm{~ns}$ with an RMS of $0.849 \mathrm{~ns}$ 
4-22 - An ideal linewidth is represented in the graph on top, where a wavelength $\lambda 0$ occurs at a single instant in time t 0 . In reality, a wavelength $\lambda 0$ occurs over a small range of time $\Delta \mathrm{t} 0$ which represents the linewidth of the laser.

4-23 - The Voigt profile is a result of collisional broadening (described by a Lorentzian profile) and a Gaussian Doppler profile which appears somewhat polynomial [13]

5-1 - Block diagram of test setup used to monitor wander of the VT-DBR optical frequency sweep. This setup is similar to the jitter measurement setup; the only devices under test in this experiment are gas absorption reference cells.

5-2 - An actual wavelength sweep in the range of the quad gas cell. The local minima which are labeled denote absorption peaks. These peaks have well-defined wavelengths associated with them.

5-3 - The calibration algorithm takes raw sweep data as an input and first removes hold points based on the data valid vector. This signal is then low-pass filtered to remove high frequency noise. Finally, absorption peaks are identified and assigned a wavelength [14]. A linear regression is performed on the wavelength vs. time data to obtain the calibration parameters: optical frequency step and offset wavelength

5-4 - Sweeps acquired at initial self-calibration, 10 minutes, 1, 2, 4, and 8 hours after. It is noted that the beginning of each sweep rises dramatically in amplitude over time. It is very easy to identify absorption peak drifts as well.

5-5 - The blue sweep was recorded directly following a self-calibration and the red sweep was recorded 8 hours later. There is a clear shift of the absorption peaks with respect to time. A deviation in amplitude is also noted.

5-6 - Once each absorption peak has been identified in time, these wavelength, time coordinates are plotted to form a linear regression model of the optical frequency sweep. This figure shows only 5 peaks, but actual linear regression is based off of 28 absorption peaks

5-7 - The locations of absorption peaks are plotted with their corresponding optical frequency to obtain a linear regression model of the sweep. This model supplies the optical frequency step and minimum wavelength of the sweep

5-8 - Optical Frequency Step over 7 days. The fluctuations are extremely minimal and do not have any sort of noticeable pattern to them.

5-9 - The minimum sweep wavelength recorded each hour for 7 days. Data suggests that there is some mechanism controlling the somewhat periodic nature of the wavelength 
5-10 - Optical frequency step monitored over 138 hours since a self-calibration with monitoring of case temperature. No significant correlation is observed and the optical frequency step remains very consistent.

5-11 - Minimum Wavelength of linear fit monitored over 138 hours following a selfcalibration. Strong correlation with case temperature is observed.....

5-12 - Another data set displays similar behavior. The minimum sweep wavelength is directly correlated with ambient case temperature

5-13 - Minimum wavelength recorded each minute for just over 2 hours with temperature data alongside

5-14 - Optical frequency step of 2nd generation VT-DBR laser system over 38 hours of data collection. A highly stable optical frequency step is observed over the data collection period as in the previous module.

5-15 - Wavelength wander of 2nd generation VT-DBR laser system over 38 hours of data collection. The new module exhibits significant performance improvements over the first VT-DBR system tested. No correlation with case temperature is observed

5-16 - The linear regression of a wavelength sweep ultimately provides the slope or optical frequency step of the sweep and the minimum wavelength or offset wavelength. A large range of wavelength offset is observed with regard to temperature while the slope stays very consistent, deviating only a small amount

5-17 - Linear Regression performed on a data set of wavelength vs. ambient or case temperature. This yields a temperature coefficient of approximately $90 \mathrm{pm}$ per degree Celsius.

5-18 - Linear Regression performed on a data set of wavelength vs. ambient or case temperature. This yields a temperature coefficient of approximately $97 \mathrm{pm}$ per degree Celsius 


\section{INTRODUCTION}

The first monolithic GaInAsP/InP wavelength tunable laser diode was developed nearly 30 years ago in 1985 [1]. Since then, single-chip tunable laser technologies have advanced significantly, expanding fields of optical communication (for which they were first developed) and biomedical imagining to faster speeds and higher resolutions. As fiber optic applications demand higher performance, it will be necessary for laser technology to meet these demands. High-speed, tunable lasers are a promising option to expand and increase the abilities of current fiber optic technologies.

Tunable lasers benefit from the ability to modulate their output wavelength in some controlled manner. Depending on the application, a tunable source may be optimized for output power, modulation speed, spectral linewidth, or output wavelength range. The Vernier-Tuned Distributed Bragg Reflector (VT-DBR) akinetic laser source represents a new generation of high-speed, tunable swept lasers [1].

A host of fiber sensing applications benefit from the VT-DBR with regard to spectral linewidth, output power, output range, sweep repetition rates, and simple control over these and other parameters. 


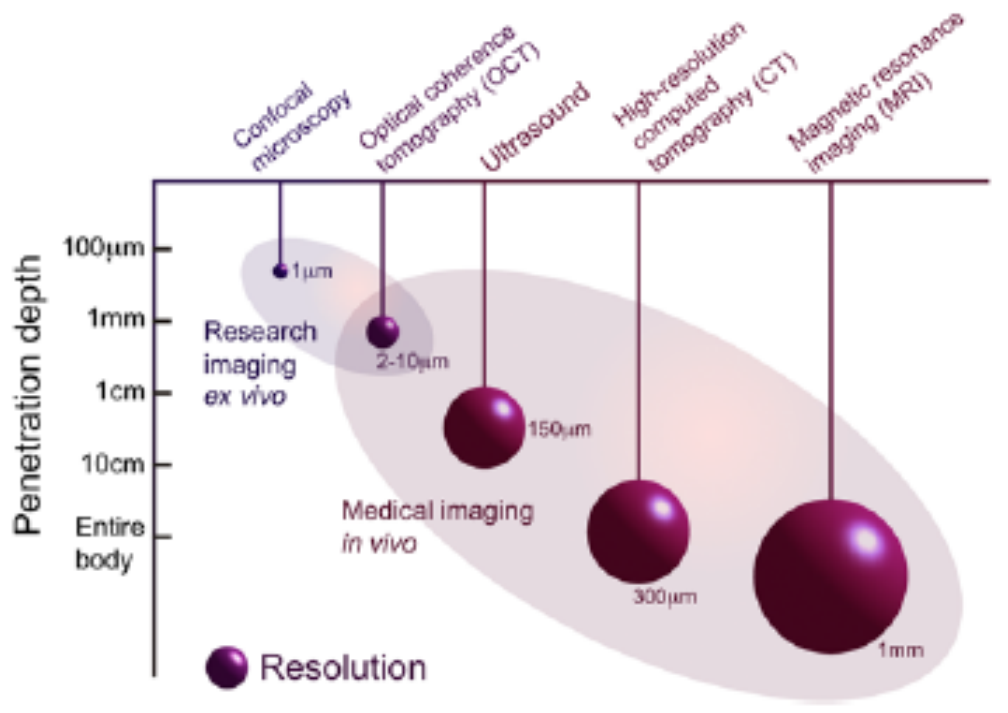

Figure 1-1 - A comparison of established biomedical imaging techniques displaying the unique market niche of OCT [8].

\subsection{Biomedical Imaging: Optical Coherence Tomography (OCT)}

Demonstrated for the first time in the early 1990s, OCT is an imaging technique used for translucent and partially opaque materials [2]. OCT is often used for biomedical imaging where it offers sub-micrometer isotropic resolution images. Applications include cardiology, where arterial walls may be mapped, optometry, dermatology, and early cancer detection. OCT may be thought of as optical ultrasound, where highly coherent light is used instead of sound. As shown in figure 1-1, OCT may be performed in or ex vivo, offering a dynamic, minimally invasive imaging modality that offers extremely high resolution imaging.

As OCT has matured over the past two decades, several methods have been developed with unique advantages. Among these are source-swept OCT (SSOCT) where a broadband, swept light source is required. Figure 1-2 shows a basic SSOCT system block diagram, implemented as a fiber-coupled Michelson interferometer. The sample arm is used to image a specimen (e.g. an artery, tooth surface, or skin). Also known as 
Fourier Domain OCT (FDOCT), this method has shown to exhibit a better sensitivity advantage over traditional time domain OCT [3]. The VT-DBR is an ideal source for this approach and has demonstrated success in achieving high resolution images of the human body [4].

Phase sensitive OCT (PhOCT), in which interferometer phase variations are monitored to obtain sub-nanometer isotropic resolution images, also benefits from a swept light source such as the VT-DBR. In PhOCT, the stability of the phase is critical to achieving high resolution images or movements. Previous results have also demonstrated success with the VT-DBR, recording sub-picometer sensitivity [5].

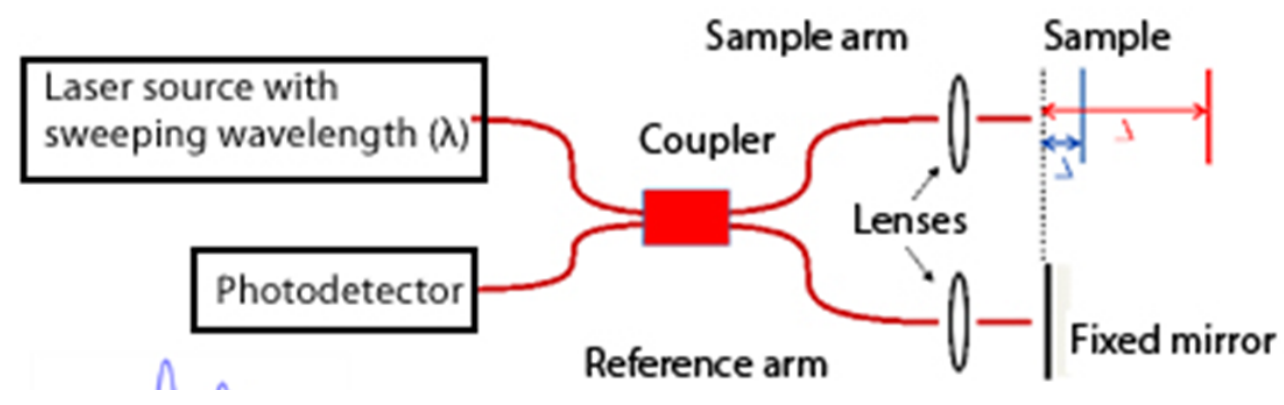

Figure 1-2 - A typical SSOCT test setup. There is a sampling output (to the photo detector) and two sample arms, one as a reference with a fixed mirror [8]. 


\subsection{Fiber Bragg Grating (FBG) Sensors}

Another fiber sensing application which benefits from a highly stable optical frequency sweep is fiber Bragg grating sensing. Fiber Bragg gratings are popular optical sensors. They are employed in numerous applications including measurement of temperatures in highly volatile environments, monitoring of strain on a building during an earthquake, or velocities and other metrics of high-speed railway trains [6]. FBGs are rugged, compact, electrically and optically passive, and may be placed serially with up to 80 sensors in a single fiber.

A fiber Bragg grating is composed of Bragg mirrors in a periodic structure. This isolates and reflects a single wavelength (the Bragg wavelength), while transmitting the rest of the incident light. As shown in figure 1-3, the Bragg grating is created by inducing small, periodic fluctuations in the indexes of refraction in a small section of optical fiber.
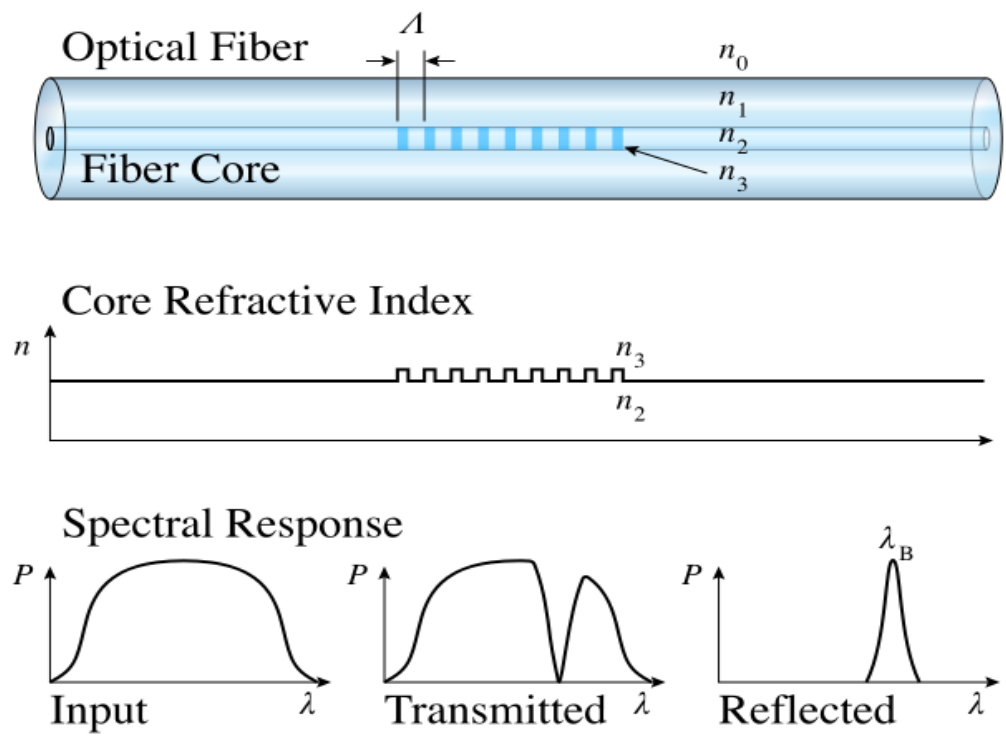

Figure 1-3 - Fiber Bragg grating (FBG) sensors reflect a characteristic Bragg wavelength, defined by the periodic refractive index changes in the fiber [47]. 
The reflected Bragg wavelength is a function of temperature, pressure, and strain on the sensor. Therefore, variations in any of these parameters cause the Bragg wavelength to shift. Figure 1-4 exaggerates the shift of the Bragg wavelength. Typical shifts in Bragg wavelength are across a range of $\sim 5$ nanometers. The sensitivity of the Bragg wavelength depends on many factors, one being jitter in the optical source.

FBG sensing requires a light source which can output a band of wavelengths, suggesting that non-tunable, single-mode lasers are not a viable solution. Swept lasers such as the VT-DBR, however, offer many benefits for this application. Benefits include high-speed sweep repetition rates, a large sweep range, simple tunability, low power output, and the ability to multiplex up to 80 sensors in a single fiber.
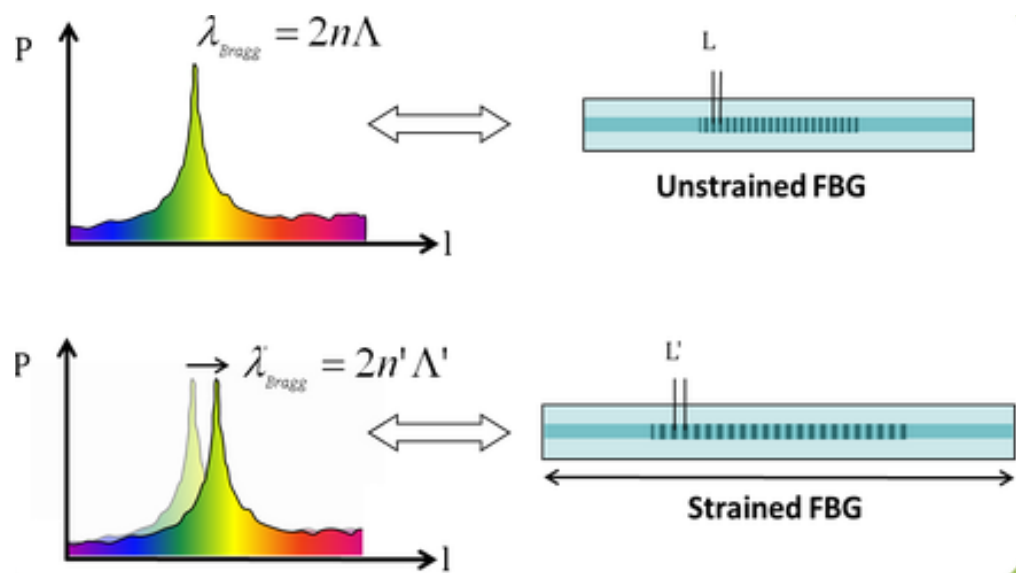

Figure 1-4 - Varying temperature, pressure, or strain on an FBG causes the Bragg wavelength to shift due to a shift in the grating period [49]. 


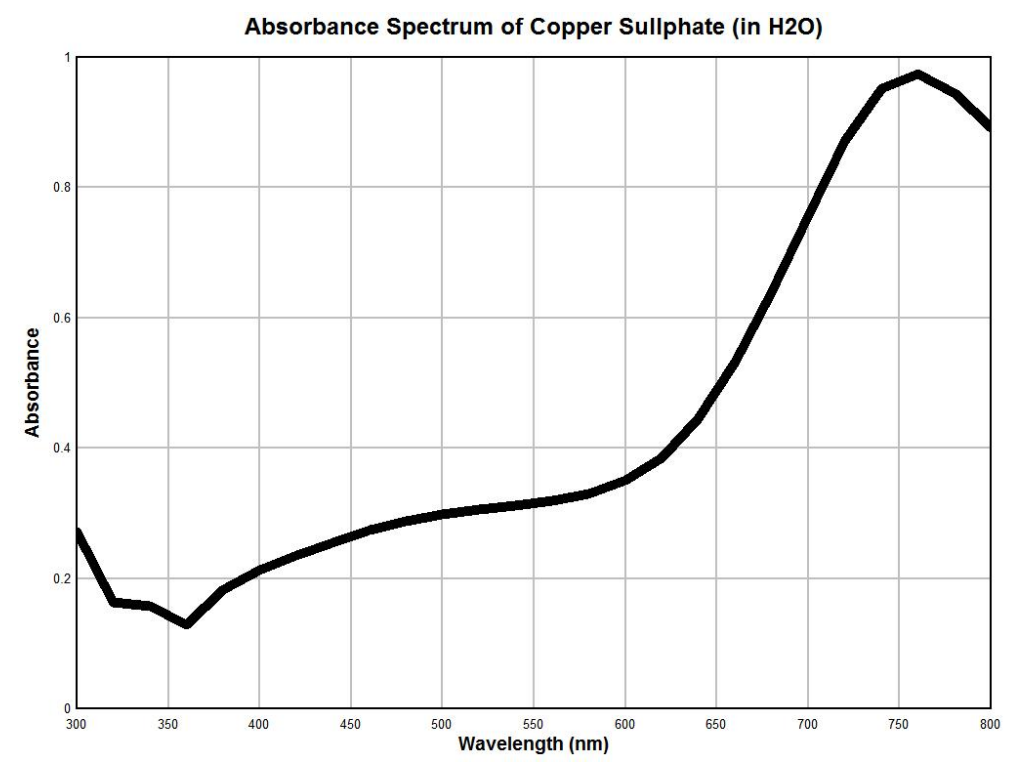

Figure 1-5 - An example absorption spectrum of Copper Sullphate in water. The lower wavelengths are not absorbed as compared to the higher wavelengths [48].

\subsection{Absorption Spectroscopy}

Absorption spectroscopy is the study of how matter interacts with, absorbs, and transmits light. Spectroscopy represents a significant application of fiber optic sensing.

All physical matter exhibits a particular response characteristic to incident light. This response is called the absorption spectrum of a material and is unique. This characteristic may be thought of as a fingerprint or other unique identifier. While this absorption spectrum spans the entire electromagnetic spectrum, absorption spectroscopy generally utilizes the visible and infrared subsets of the entire spectrum.

Spectroscopy is used in a wide range of areas, from biomedical imaging to astronomy. Spectroscopy is a way to determine the presence of a gas, liquid or solid based on its chemical signature.

In order to obtain an absorption characteristic that spans a large range of the visible spectrum and thereby carries more information, a broadband source is generally required. Again, the VT-DBR provides benefits, especially in laser absorption 


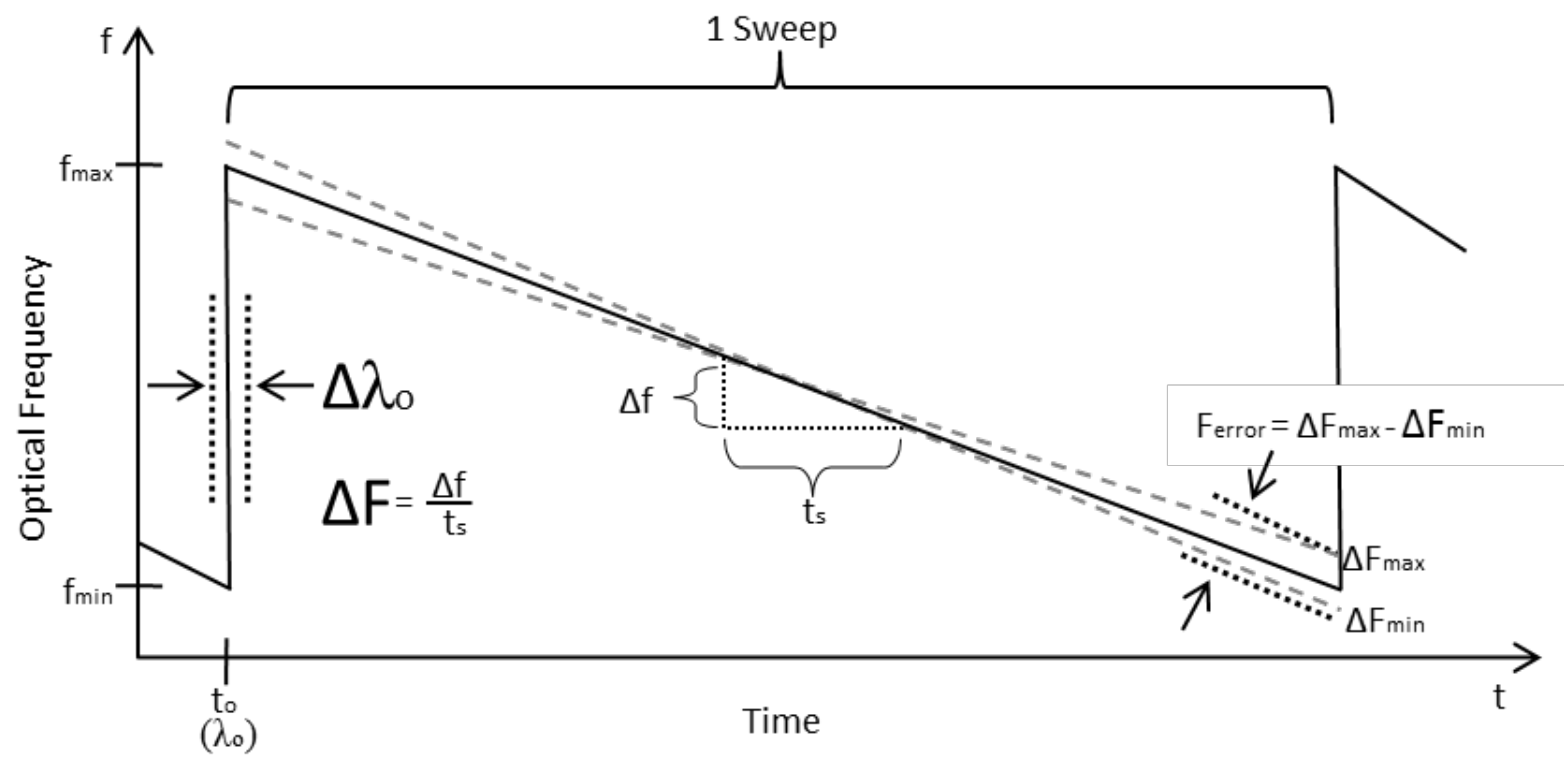

Figure 1-6 - A laser sweep begins with an initial optical frequency at initial time $\left(\mathrm{t}_{\mathrm{o}}\right)$ and sweeps from $f_{\max }$ to $f_{\min }$ at a rate equal to the optical frequency step, $\Delta \mathrm{F}$.

spectroscopy (LAS), which provides highly accurate quantitative measurements of gas atoms and molecules. It should be noted that present VT-DBR models have limited optical frequency output ranges which limits their usefulness in LAS.

\subsection{Purpose of Study}

VT-DBR lasers have shown novel performance in fiber optic sensing applications. Benefits of the VT-DBR include narrow linewidth, ultra-fast sweep rates, unprecedented sweep linearity, and highly accurate sweep-to-sweep wavelength repeatability, all of which may be user-controlled [6]. Stability of the optical frequency sweep is another key performance characteristic.

Presently, the stability of the wavelength or optical frequency sweep of the VTDBR has not been undertaken. To ensure that the VT-DBR is a reliable source for OCT, FBG sensing, and other fiber optic applications, understanding the accuracy, stability, and robustness of the laser's sweep is crucial. Benefits of a highly-stable linear optical 
frequency sweep include higher resolution imaging, improved phase stability [5], higher fidelity sensor measurements, simpler data acquisition, and consistent measurement results.

This project investigates the sweep stability of the VT-DBR laser source. Metrics of jitter and wander are quantified to inform on the short- and longer-term stability of the wavelength sweep.

An ideal VT-DBR has a specific optical frequency or wavelength step and welldefined minimum and maximum wavelength output during a sweep. These metrics describe the linearity of the device at the time of calibration, as shown in figure 1-6. These values, however, may deviate over short and long periods of time. This work investigates these deviations and the temporal stability of the laser source.

\subsection{Thesis Summary}

Chapter 2 reviews basic laser physics and describes the tuning mechanism of the VT-DBR. Chapter 3 establishes a common understanding for the metrics of test in this project: jitter, wander, and sweep stability.

Chapters 4 and 5 provide data on jitter and wander characterization of the laser. Jitter is reported along with wander over several periods of time. A unique, external calibration method using a gas absorption cell is detailed.

Chapter 6 shares conclusions with regard to the laser as well as applications to fiber sensing. Chapter 7 outlines experiments and projects for future exploration of the VT-DBR. 


\section{VT-DBR LASER THEORY}

A review of basic laser physics and a formal overview of the VT-DBR laser provide important foundations for this work. Fundamentally, the VT-DBR is an SG-DBR laser with several distinguishing features as a result of its unique construction and Vernier-effect operating mechanism.

\subsection{Laser Physics Background}

To produce optical output, a laser requires 3 critical components. First, a gain medium or lasing material is required in which a state of stimulated emission may be achieved. The VT-DBR uses a semiconductor for this material, but a gas, liquid, or other solid with the right properties may also be used. Second, an optical resonator must be placed around the gain medium to create a source of feedback in the system. This optical resonator is composed of two parallel mirrors, one of which is partially-transmissive to allow light to escape the laser cavity. Last, a laser requires a source of energy, referred to as a pump. The pump injects electrical or optical energy into the lasing medium to induce stimulated emission [7].

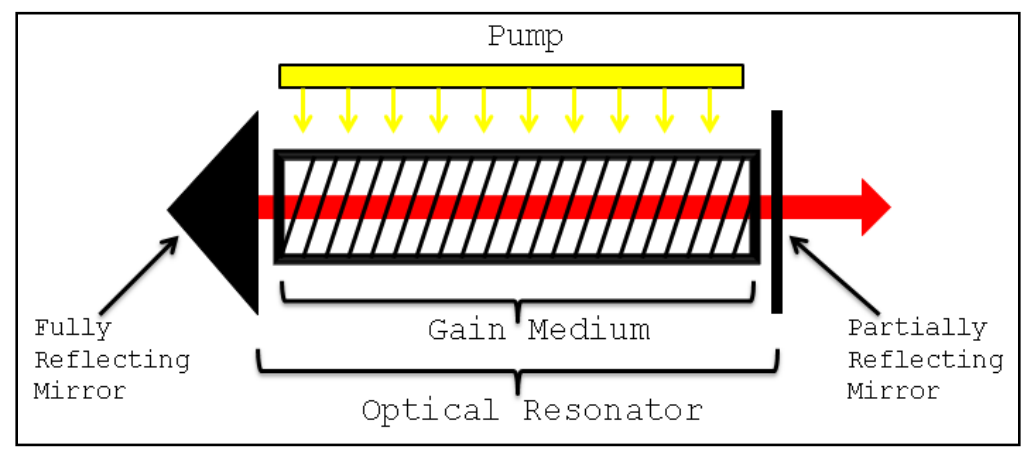

Figure 2-1 - A laser is composed of 3 fundamental components: an optical or electrical energy pump, an optical resonator composed of a fully reflecting mirror and a partially reflecting mirror, and a gain medium where stimulated emission takes place. 
In a typical light source such as an LED or incandescent bulb, photons are emitted when electrons spontaneously transition from a high energy level to a lower energy level. In such a source, energy is supplied to the active medium (i.e. a filament in an incandescent light bulb) to raise electrons to an excited energy level. These excited electrons spontaneously transition to a lower energy level, emitting a photon with energy approximately equal to the difference in energy levels.

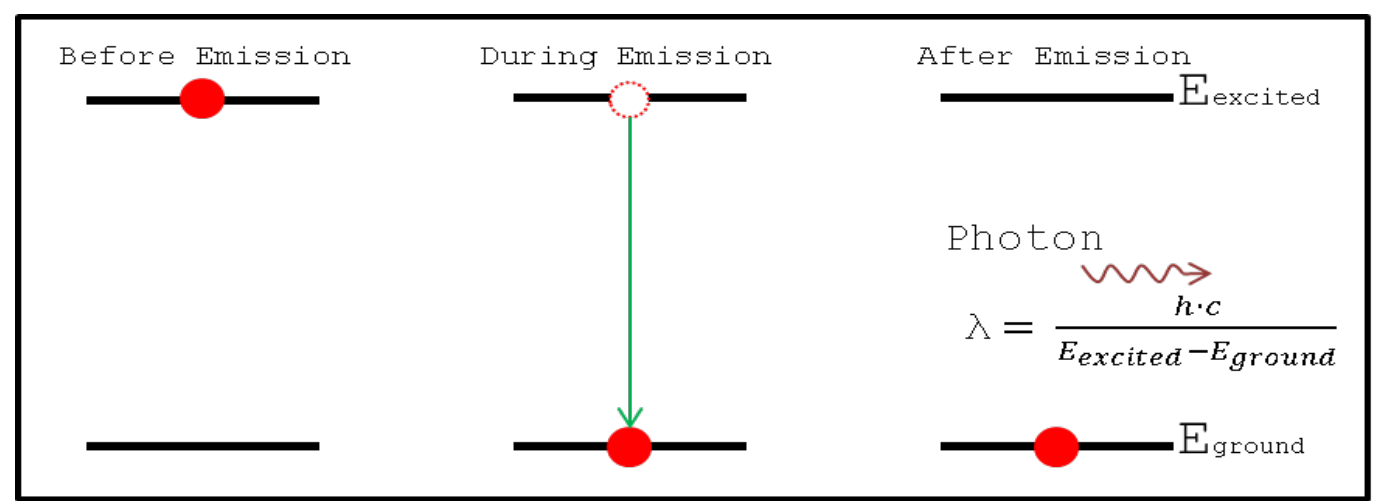

Figure 2-2 - Spontaneous emission is the light-producing mechanism in most light sources. An atom in an excited state transitions to a lower state spontaneously emitting a photon in the process.

In contrast, the excited electrons in a laser medium move to a ground energy level not randomly, but when they are stimulated by other photons that collide with them.

Stimulated emission occurs when a photon interacts with an excited electron, causing the electron to transition to a ground energy level. As a result, two photons are emitted from the electron as it transitions to a lower energy level. Both photons are in phase and have identical energies and, thus, wavelengths. 


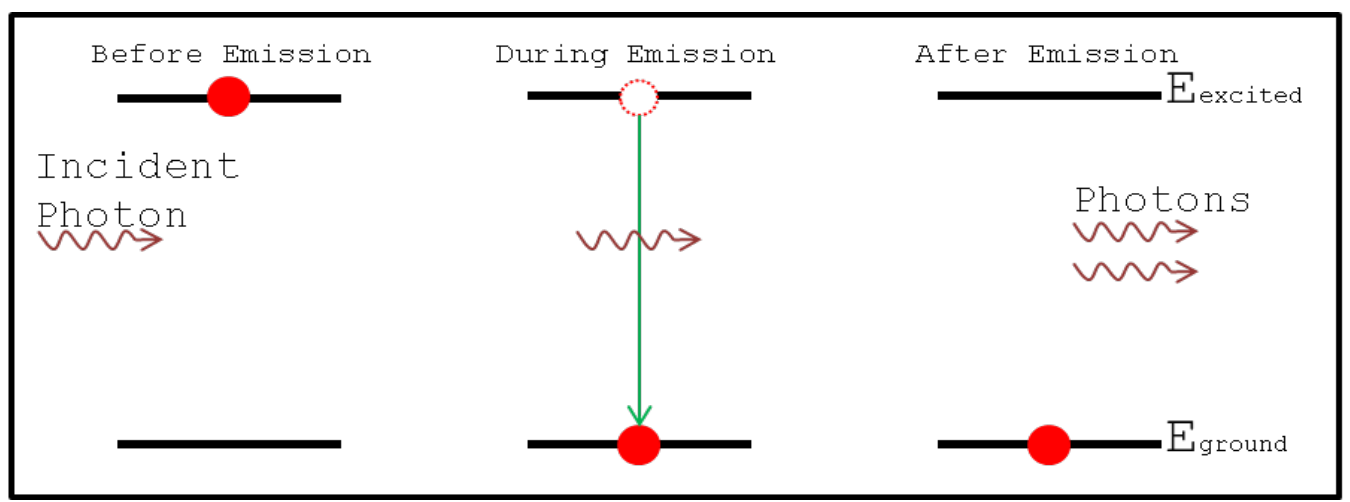

Figure 2-3 - In stimulated emission, a photon causes an excited atom to transition to its ground state, emitting two phaselocked photons of identical wavelength.

In order to ensure that stimulated emission may occur, a critical state of population inversion must exist inside the active gain medium [7]. Population inversion is the state when more electrons exist in an excited or metastable energy level than the ground level. This ensures that when a photon travels through the laser cavity, there is a high, nearly certain probability that the photon will interact with an excited atom, producing two more photons and continuing the process light amplification by stimulated emission radiation (LASER acronym). Figure 2-4 illustrates the laser amplification cycle where pumping energy keeps more electrons in an excited or metastable

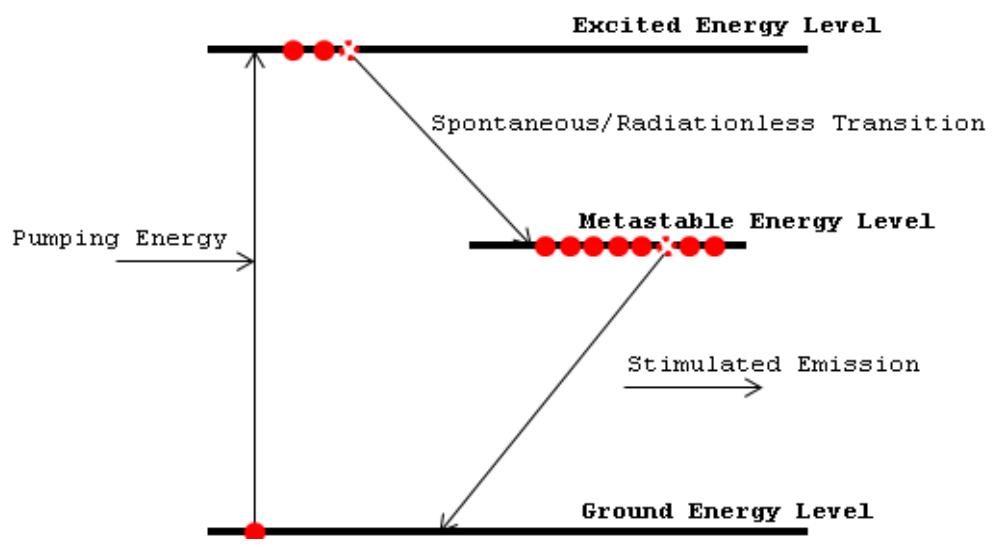

Figure 2-4 - Energy level diagram of a 3-level laser showing a state of population inversion where more electrons are excited than in the ground state. 
energy level than the ground level. The process of stimulated emission occurs when a photon in the optical resonator of the laser interacts with an excited atom, releasing two photons of highly coherent or in-phase light.

\subsection{VT-DBR Tuning}

The laser theory described in the previous section provides the foundation for a new class of sampled-grating distributed Bragg reflector (SG-DBR) lasers. SG-DBR lasers are beneficial because they have the ability to produce a highly coherent single longitudinal mode that is electronically tunable. The SG-DBR is the foundation for the VT-DBR laser.

A dielectric or Bragg mirror provides the basis for tunable laser output. A Bragg mirror is created by placing two materials of varying refractive index next to one another. By periodically concatenating Bragg reflectors, a distributed Bragg mirror is constructed. The reflector or grating acts as an optical filter and only allows certain modes to propagate from the active region of the laser. This gives the laser a very small linewidth the ability emit over a very small band of wavelengths. The central wavelength depends only on the refractive indices of the grating and the length of the laser cavity. By applying a current to the different semiconductor segments of the laser cavity, small variations in refractive index are induced, altering the output wavelength of the laser.

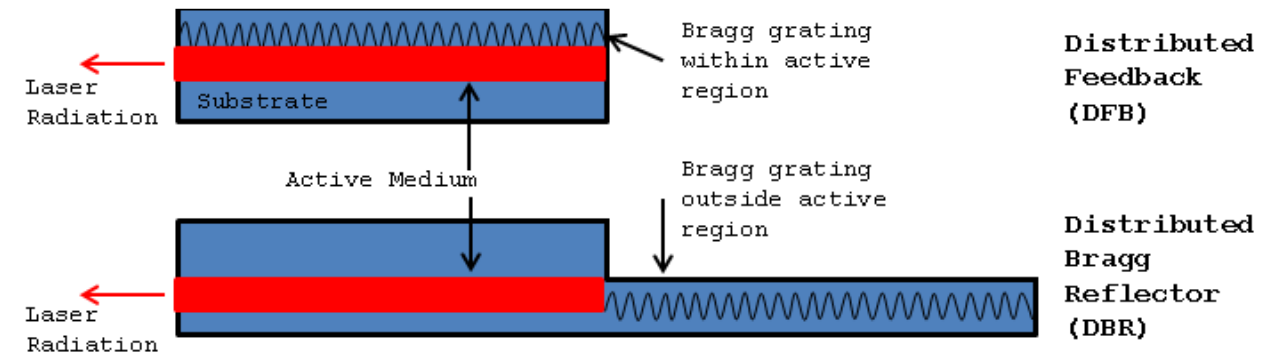

Figure 2-5 - Bragg gratings are used to create distributed feedback in a DBR grating. These gratings are composed of materials of varying refractive index. 
This is the mechanism responsible for controlled output wavelength tuning.

As shown in figure 2-6, the SG-DBR has 5 control currents to perform wavelength tuning: back mirror, gain amplifier, phase, front mirror and semiconductor

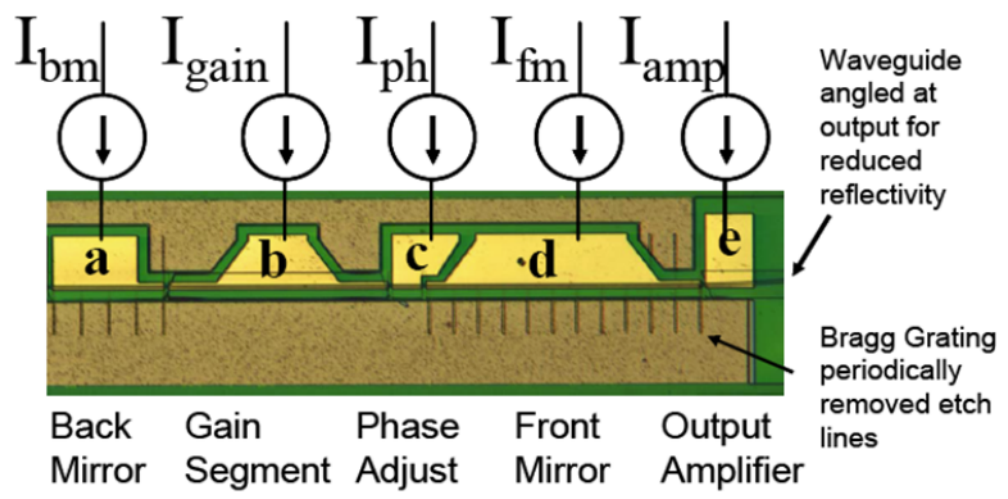

Figure 2-6 - The VT-DBR has a sampled grating distributed Bragg reflector structure controlled by 5 separate currents for wavelength tuning [6].

optical amplifier (left to right in figure 2-6). The front mirror (FM), back mirror (BM), and phase (PH) sections of the SG-DBR structure are responsible for wavelength selection [4]. The drive currents of these three frequency-dependent sections induce a change in refractive index of the mirror sections and cause the laser to output a specific wavelength. The semiconductor optical amplifier (SOA) and gain $(\mathrm{G})$ sections allow control of the power output of the laser [8]. 

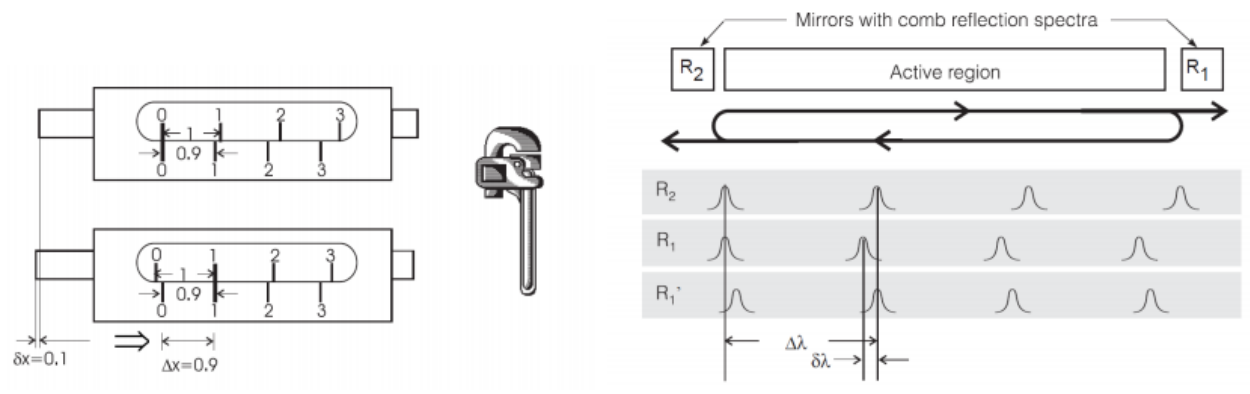

Figure 2-7 - The Vernier effect is most often used for precise distance or angle measurements, but is exploited by the VT-DBR in a unique fashion [1].

The VT-DBR is an SG-DBR style laser leveraging the Vernier effect, traditionally used for accurate, high resolution distance measurements using calipers. In distance measurements, the Vernier effect works by offsetting a primary scale with a secondary scale that has a pitch difference (i.e. $10 \%$ pitch difference as shown in the left side of figure 2-7). Vernier tuning in the VT-DBR laser is derived from the distributed Bragg reflectors on either side of the active gain medium of the laser cavity. Each of these distributed Bragg reflector structures provides a comb reflection spectrum, as shown in the right of figure 2-7 [1]. By ensuring a similar pitch shift in one of the reflectors and the proper cavity width, smaller wavelength tuning steps are achieved in the VT-DBR.

The VT-DBR has also been coined as the Akinetic Swept Laser, where "akinetic" refers to the absence of any physical movement or momentum inducing parts in the tuning mechanism. Other, non-SG-DBR tunable sources such as tunable external-cavity lasers (ECL) or laser arrays with micro-electro-mechanical systems (MEMS) for tuning all suffer from fundamental limitations on tuning speed [4]. As shown in figure 2-8, the VT-DBR is controlled only by electronic currents with no mechanical elements, eliminating any mechanically-induced noise. Ultimately, tuning in the VT-DBR is achieved by dynamically modulating 5 current sources. 
While performance, tuning speeds, and noise are much improved with the absence of mechanical tuning components, noise may be introduced into the system by the complex drive circuitry employed in such a laser system. The noise contributing elements include a thermo-electric cooler (TEC), which contributes thermal noise, and an FPGA which drives digital-to-analog converters and transimpedance amplifiers to modulate drive currents. These all induce shot noise which directly correlates to noise in output wavelength and power. Wavelength jitter and wander may ultimately be caused by one or more of these elements along with random noise in the laser chip itself.

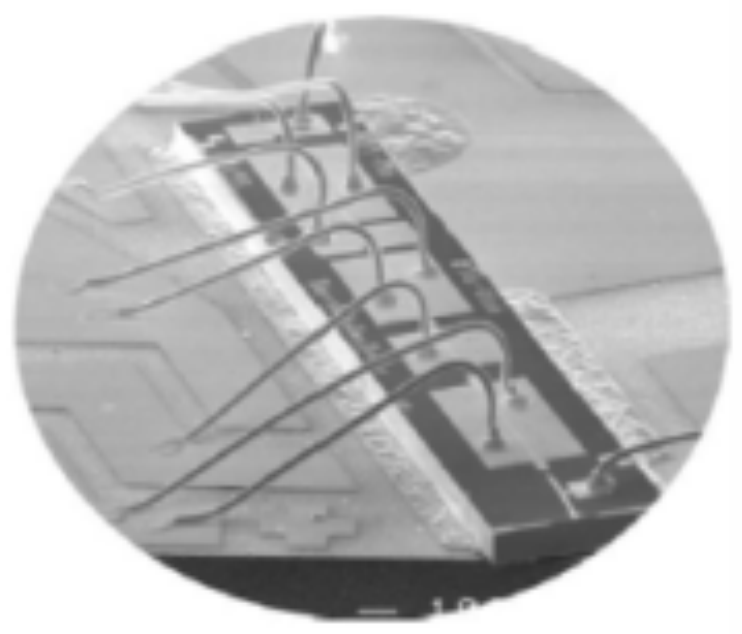

Figure 2-8 - The VT-DBR is contained on a single monolithic chip with 5 control currents to conrol the front mirror, back mirror, phase, gain, and semiconductor optical amplifier [2]. 


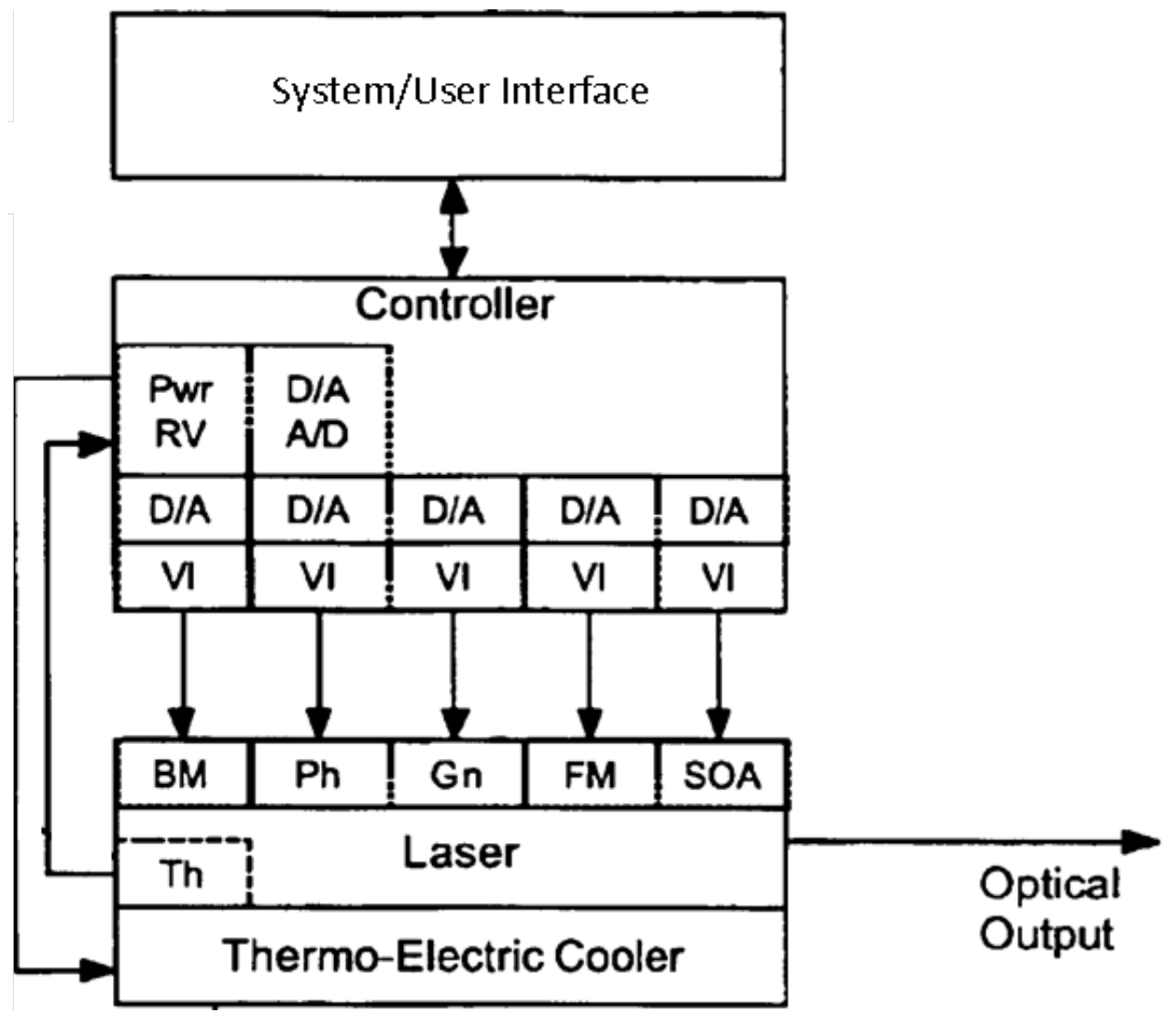

Figure 2-9 - The VT-DBR Akinetic laser system contains complex drive circuitry and algorithms as well as a Thermo-Electric Cooler, all of which contribute noise to the system. The TEC attempts to regulate the temperature of the laser chip, but induces some amount of thermal noise into the system. The digital-to-analog converters (D/A) and trans-impedance amplifiers (VI) have a shot noise limit which limits the noise induced into the laser segments and, thus, their output wavelength selectivity and power. This noise ultimately contributes to the short- and long-term stability of the optical sweep [50]. 


\subsection{VT-DBR Laser System Performance Advantages}

Due to the all-electronic tuning mechanism, the VT-DBR system exhibits enhanced performance over other solutions.

The VT-DBR exhibits the unique advantage of an adjustable coherence length [4] - the distance over which a coherent wave maintains a specified coherence. Often, lasers are advantageous due to their exceptionally long coherence length, however it may be desirable to lower coherence length in some applications [9] - something which is usercontrollable in the VT-DBR.

Another advantage of the VT-DBR system is the ability to sweep across nonconjoined wavelength or optical frequency regions - a result of its all-semiconductor structure. Figure 2-10 illustrates this concept, which is not possible with any other swept laser source. This ultimately provides increased information for absorption spectroscopy or improved spatial resolution in several applications including OCT [4].

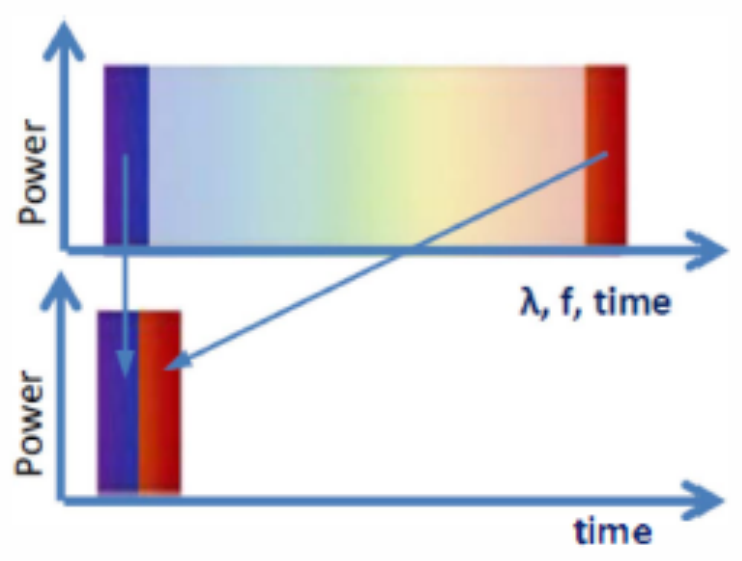

Figure 2-10 - It is advantageous in some applications to quickly sweep across two, nonconjoined sweep ranges. The VT-DBR has the ability to output such a non-contiguous laser sweep rapidly, which is impossible with other swept lasers [4]. For example, a sweep of 50 $\mathrm{nm}$ at a center wavelength of $1310 \mathrm{~nm}$ may take place followed rapidly by another $50 \mathrm{~nm}$ sweep at a $1550 \mathrm{~nm}$ center wavelength. 
The VT-DBR allows for custom power profiles to be implemented with simple current control. A user may find this advantageous for a given application, especially if the sweep power is amplified or attenuated significantly at a given wavelength. It is possible to "power level" the laser with a custom profile to remove such power variations in a system.

Unlike other tunable sources with mechanical components, the sweep repetition rate of the VT-DBR may be altered immediately and over a large range in the VT-DBR.

One of the fundamental benefits of the VT-DBR system is user-controlled through software, allowing a high level of programmability and control over laser sweep parameters. A user has the ability to select a custom power versus wavelength profile (Gaussian, flat, or custom for power leveling), duty cycle, sweep speed, the minimum and maximum wavelengths of a sweep, coherence length, number of measurement points, and sweep direction along with many other parameters.

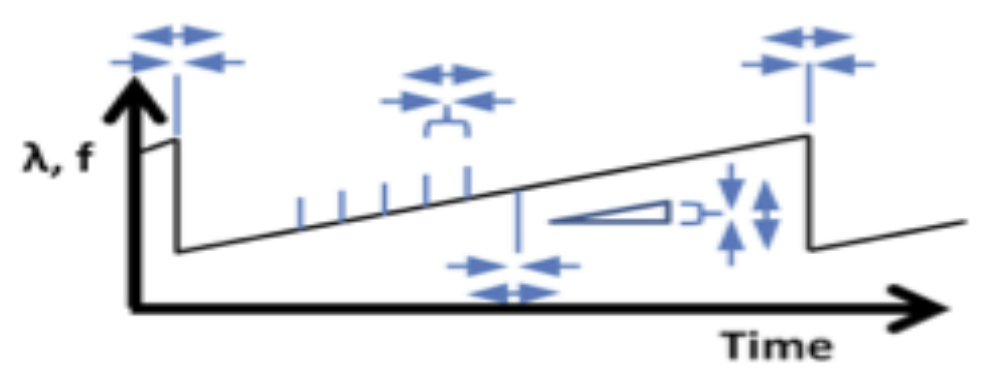

Figure 2-11 - The VT-DBR system allows for a high degree of sweep programmability, as illustrated by the blue arrows. A user may customize parameters such as number of measurement points, sweep width, coherence length, sweep speed, duty cycle, power profile, and others [4]. 


\section{DEFINING SWEEP STABILITY}

The purpose of this project is to investigate one of the unique aspects of the VTDBR laser that makes it successful in OCT and other fiber sensing applications: the stability of the linear optical frequency sweep. Before we explore stability and quantify performance of the source, a common understanding of sweep stability should be established.

Throughout this work, the sweep of the laser is interchangeably referenced in terms of optical frequency or wavelength. In general, optical frequency is used to describe the slope of the laser sweep, while wavelength is used to discuss the offset of the linear laser sweep. It is noted that the laser sweeps linearly in frequency. We may easily convert between optical frequency $(\mathrm{Hz})$ and optical wavelength (meters) by a factor of the speed of light in a vacuum $(\mathrm{c}=29979258 \mathrm{~m} / \mathrm{s})$ :

$$
f \times \lambda=c
$$

All calculations in this work are initially completed in the optical frequency domain before converting to a wavelength equivalent. Results reported in wavelength are often times more intuitive depending on the application.

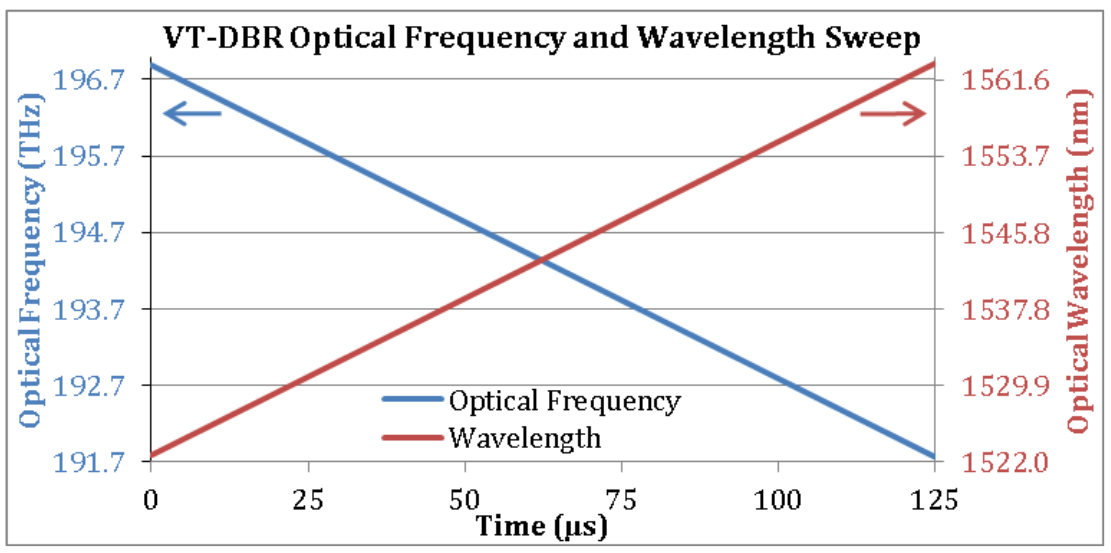

Figure 3-1 - A typical sweep of the VT-DBR is linear in either optical frequency or wavelength. Either is an appropriate description for the sweep of the laser. 


\subsection{Definition}

The most basic question that we seek to answer in order to characterize sweep stability is:

"how stable in time is the output sweep of the VT-DBR?"

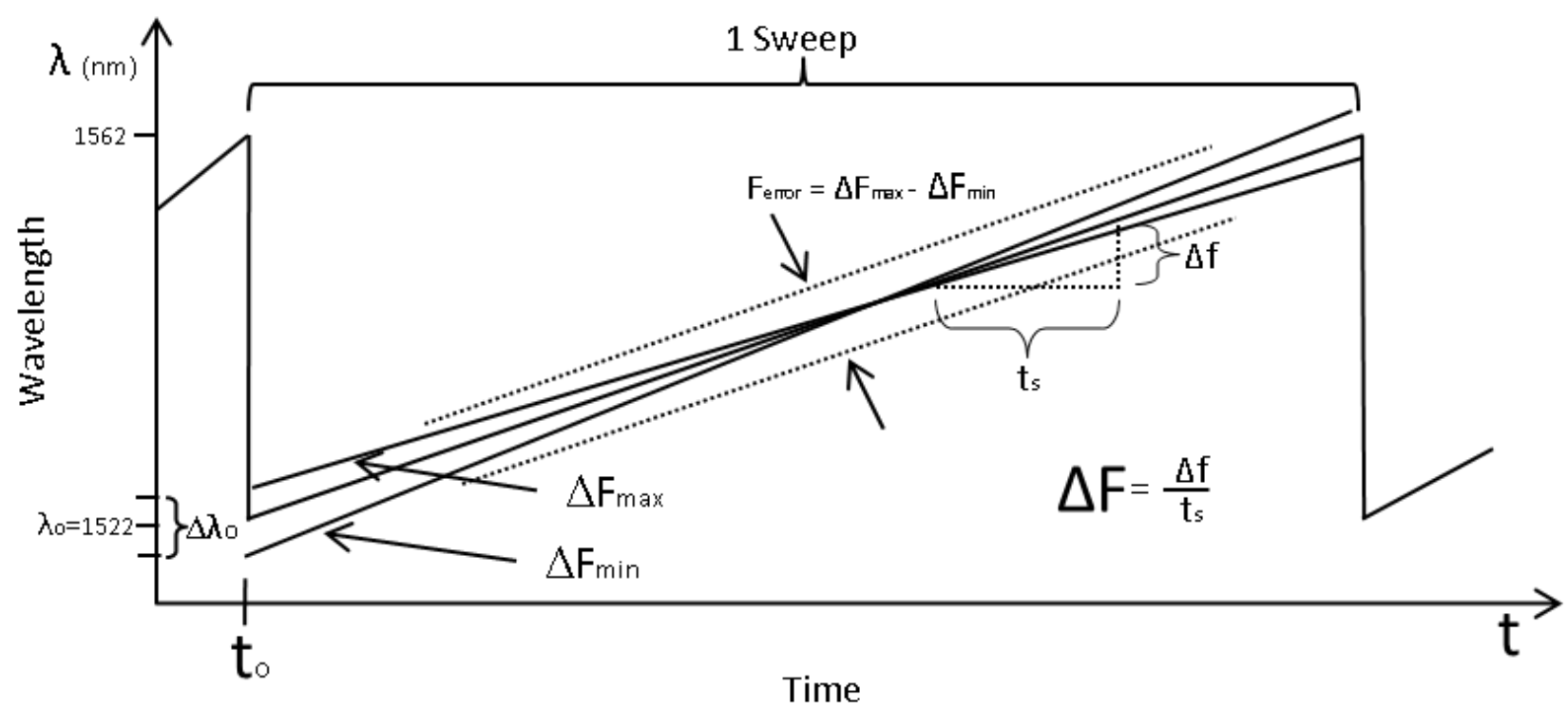

Figure 3-2 - An ideal laser sweep is described by its minimum wavelength, $\lambda_{0}$ (offset) and optical frequency step (slope), $\Delta \mathrm{F}$. Stability of the optical frequency sweep is defined through deviations in linearity, $\mathrm{F}_{\text {error }}$, and minimum sweep wavelength, $\Delta \lambda_{0}$.

An ideal laser output is perfectly consistent in absolute optical frequency over time and perfectly stable in linearity as well. A sweep can most easily be described by two metrics: the optical frequency step (slope) and minimum sweep wavelength $\lambda_{\text {min }}$, as shown in figure 3-2.

The optical frequency step is a metric describing the slope of the optical frequency sweep. The VT-DBR operates on a $400 \mathrm{MHz}$ sample clock, which means that the laser output wavelength may only step up or down every $\frac{1}{400 \mathrm{MHz}}=2.5 \mathrm{~ns}$. Therefore, the optical frequency step is most often reported in $\mathrm{MHz}$ per $2.5 \mathrm{~ns}$, instead of per second 
or per nanosecond.

The smallest increment of the optical frequency step is about $100 \mathrm{MHz}$ per $2.5 \mathrm{~ns}$. Physically, this means that the laser's output increases or decreases in optical frequency by $100 \mathrm{MHz}$ every $2.5 \mathrm{~ns}$ of a sweep. For those more accustomed to wavelength units, this corresponds to a wavelength change of approximately

$0.833 \mathrm{pm}$ per $2.5 \mathrm{~ns}$ - the wavelength step of the laser. Optical frequency step and wavelength step may be interchangeably used as well, where $v=c=299,792,458$ meters $/$ second and $\lambda=1550 \mathrm{~nm}$ :

$$
\begin{gathered}
\Delta \lambda=-\frac{\Delta f \cdot \lambda^{2}}{v} \\
\Delta f=-\frac{\Delta \lambda \cdot v}{\lambda^{2}}
\end{gathered}
$$

The second parameter of interest is the minimum wavelength or offset wavelength of the sweep. This can just as easily be coined the maximum optical frequency or optical frequency offset (since wavelength and frequency metrics may be used interchangeably). This value represents the minimum wavelength that is output by the laser in a positivelysloped wavelength sweep. This can be thought of as the starting wavelength of a sweep.

If a sweep is perfectly linear, then these two parameters - optical frequency step and offset wavelength - fully describe a laser sweep.

\subsection{Definition: Jitter}

Jitter is defined as, "the short-term variations of significant instants of deviation of a timing signal from ideal positions in time" [10]. Jitter is defined for periodic timing signals, where we expect to know the amplitude of a signal at any instant purely as a function of time. In practice, we find that a signal is not always so precise and given a 
particular signal amplitude value or amplitude transition, the time at which value or transition occurs may have a range of values instead of a single, well-defined value that is always the same. We define this range of times as the peak-to-peak jitter of the signal, as can be seen in figure 3-3.

In this work, we are interested in quantifying the optical frequency jitter of the VT-DBR laser source. As seen in figure 3-1 and 3-2, we expect that at any moment in time, due to the linear nature of the frequency sweep, we may easily resolve the optical frequency that the VT-DBR outputs to a very high accuracy. This assumes that there is no jitter present in the sweep.

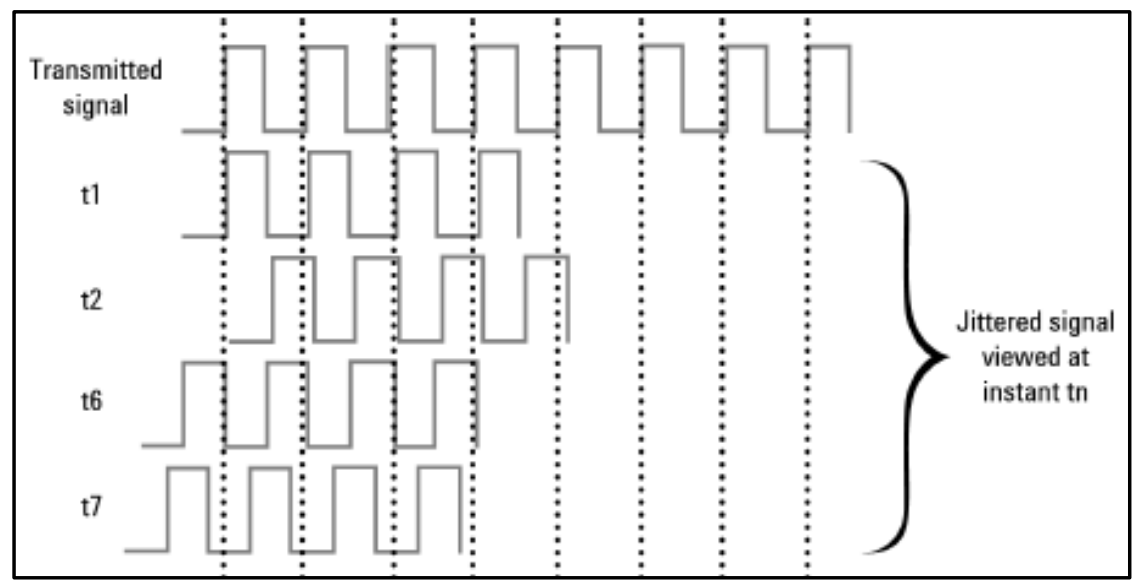

Figure 3-3 - Traditional jitter measurements are made on timing signals or clocks. These signals are well-defined and periodic in nature [10].

\subsection{Definition: Wander}

While jitter addresses short term, high frequency variations at $10 \mathrm{~Hz}$ and greater [10], wander is an equally important metric. Wander is somewhat of a compliment to jitter, encompassing variations that are sub- $10 \mathrm{~Hz}$ and often much slower. As the term implies, wander represents the amount that a signal drifts from its expected value over longer periods of time - on the order of minutes, hours, or days.

The most intuitive understanding of wander of the optical frequency or 
wavelength sweep is to imagine a single frequency/wavelength in the sweep. Ideally, this wavelength occurs at the same location in each sweep. Over time, however, this wavelength may drift away from its initial location. We say that this wavelength wanders away from its initially calibrated location. The distance that this wavelength wanders in time, optical frequency, or wavelength is recorded as the wander.

It is believed that the VT-DBR system outputs a single, completely identical sweep repeatedly for any amount of time. We seek to verify the extent to which this is true. We also seek to understand how external factor such as temperature might impact wander or drift in the laser.

\subsection{Importance of Sweep Stability}

The motivation for this project is to validate the VT-DBR system for a multitude of fiber sensing applications. The short term jitter and longer-term wander fluctuations in the laser output will ultimately define the precision with which we can trust a sensor measurement. As discussed in section 1.2, fiber sensors such as fiber Bragg gratings operate on the premise that the wavelength which they reflect (Bragg wavelength) is a function of external factors such as strain, pressure, temperature, or other environmental changes. If the wavelength of the VT-DBR source jitters or wanders at all, it will induce errors into the measurement.

It is noted that the requirements for an OCT source may differ slightly from the requirements of a source for fiber sensing applications. This is worth mentioning because the VT-DBR has shown great success already in the OCT realm. An OCT sweep, for example, may be calibrated often and the sweep must only remain stable and consistent for as long as the scan takes - less than 1 hour in general. In general, an OCT scan is more 
sensitive to jitter than wander.

In fiber sensing applications, however, the source will often be required to operate idly for days, if not weeks without calibration. Thus, it must be known whether or not the VT-DBR maintains stability over longer periods of time.

If, for example, a sensor is calibrated to a temperature of $25^{\circ} \mathrm{C}$ at $1550 \mathrm{~nm}$, but the source drifts by $200 \mathrm{pm}$ over several days, the temperature measurement will now appear to be centered at $1550.2 \mathrm{~nm}$ and the measurements will all be skewed.

\subsection{Assumptions and Adaptations Used in this Study}

Having established a common understanding of the metrics under investigation in this thesis (slope and offset of a linear optical frequency or wavelength sweep), some assumptions and adaptations are noted.

At present, several methods exist for measurement of timing jitter in mode locked lasers, but methodologies for jitter measurements on a swept laser source have not been formally established or made available to our knowledge.

We obtain an estimate for optical frequency or wavelength jitter $\left(f_{\text {jitter }}\right.$ or $\lambda_{\text {jitter }}$ respectively) by locating a wavelength in the sweep and recording the timing jitter $\left(\mathrm{t}_{\mathrm{jitter}}\right)$ of this wavelength. We may then infer optical frequency jitter through simple conversions, utilizing the optical frequency or wavelength step ( $\Delta \mathrm{f}$ or $\Delta \lambda$ respectively) of the laser:

$$
\begin{aligned}
& f_{\text {jitter }}=t_{\text {jitter }} \cdot \Delta F \\
& \lambda_{\text {jitter }}=t_{\text {jitter }} \cdot \Delta \Lambda
\end{aligned}
$$




\section{JITTER CHARACTERIZATION}

Intrinsic jitter of the VT-DBR laser system is measured using 4 different devices under test. The test setup for jitter characterization is detailed and each reference device is explained. Results are reported in peak-to-peak and RMS wavelength and optical frequency jitter. To simulate fiber sensing applications, jitter is measured using different lengths of optical fiber to observe any effects induced by long propagation times. Jitter over the entire range of the optical frequency sweep is sampled to determine if jitter is more prominent at any particular wavelengths. A 2nd generation laser is measured to compare jitter in the two units.

\subsection{Test Setup and Theory}

To measure VT-DBR jitter, the test setup in figure 4-1 was constructed. The VTDBR laser system receives commands from the user via computer Ethernet. The user specifies parameters such as sweep rate, minimum/maximum wavelength of the sweep, output power profile, duty cycle, user clock rate, and on which edges to trigger a sweep. The parameters in table 1 were selected for this work. The minimum and maximum wavelength values selected represent the maximum sweep range possible (other VT-DBR modules offer a greater sweep range of up to $100 \mathrm{~nm}$ ). The sweep rate is the minimum sweep rate which provides the most sample points per sweep (approximately 50,000 sample points). The optical frequency step is always between $100-125 \mathrm{MHz}$ per $2.5 \mathrm{~ns}$. 


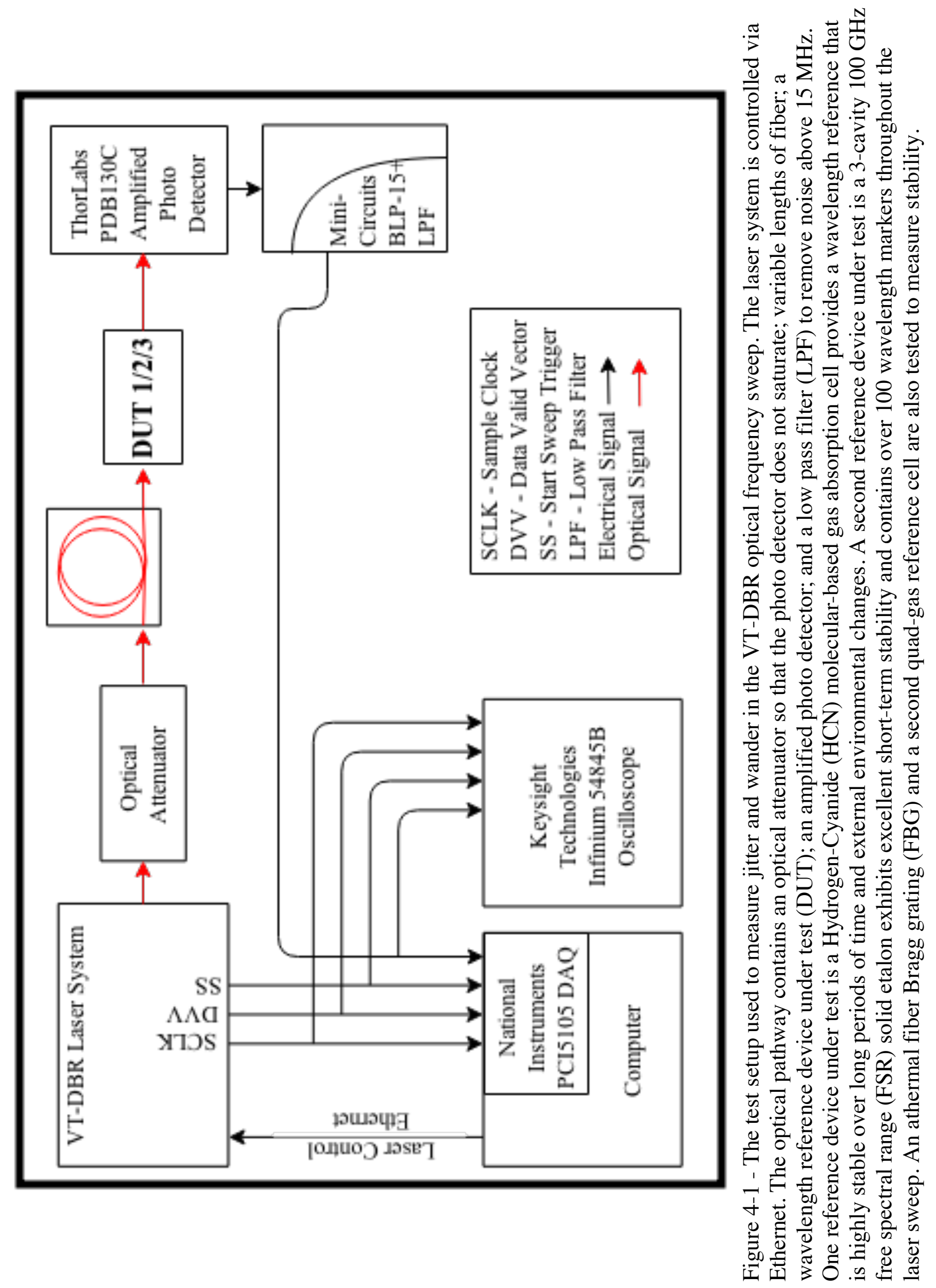




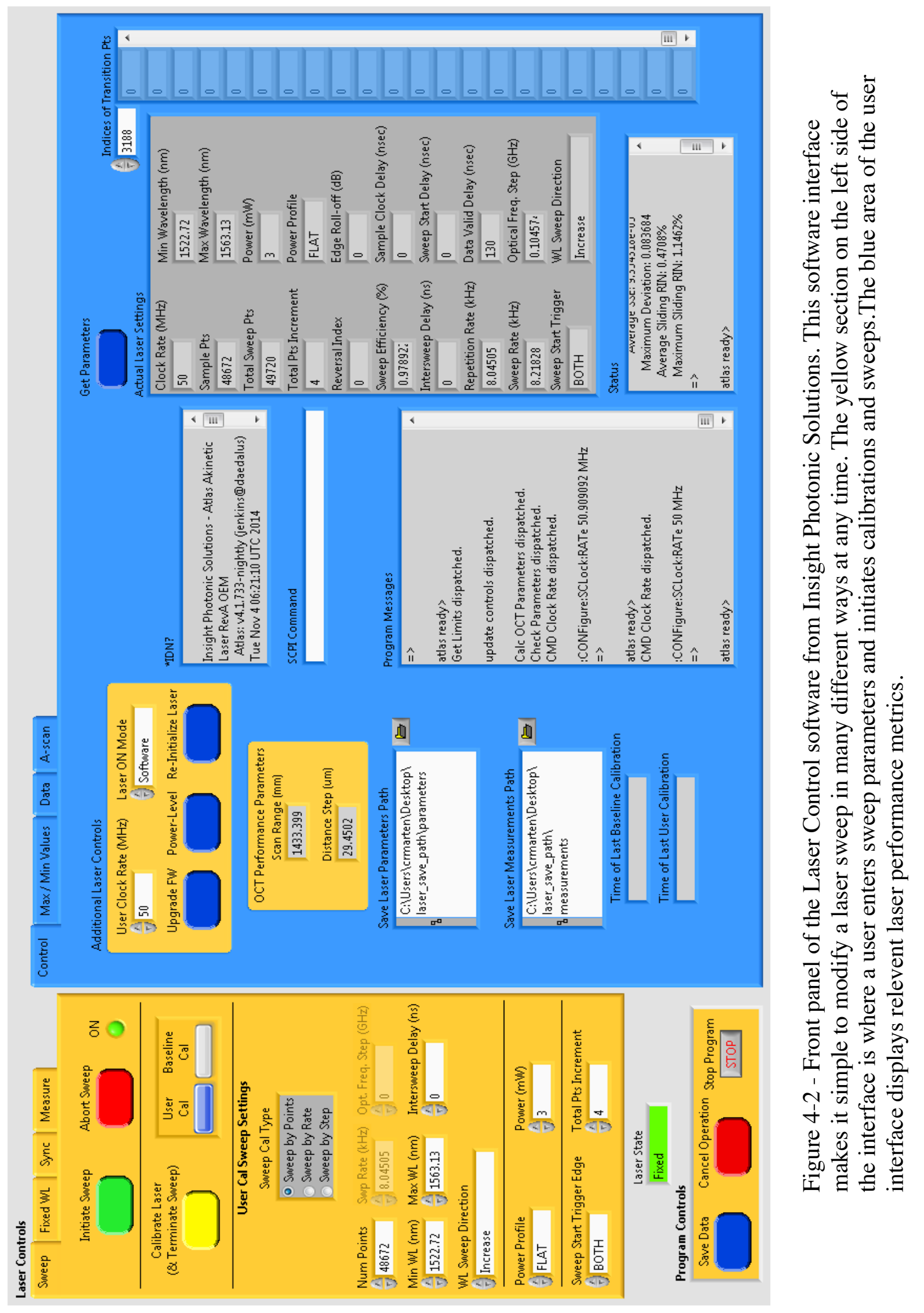




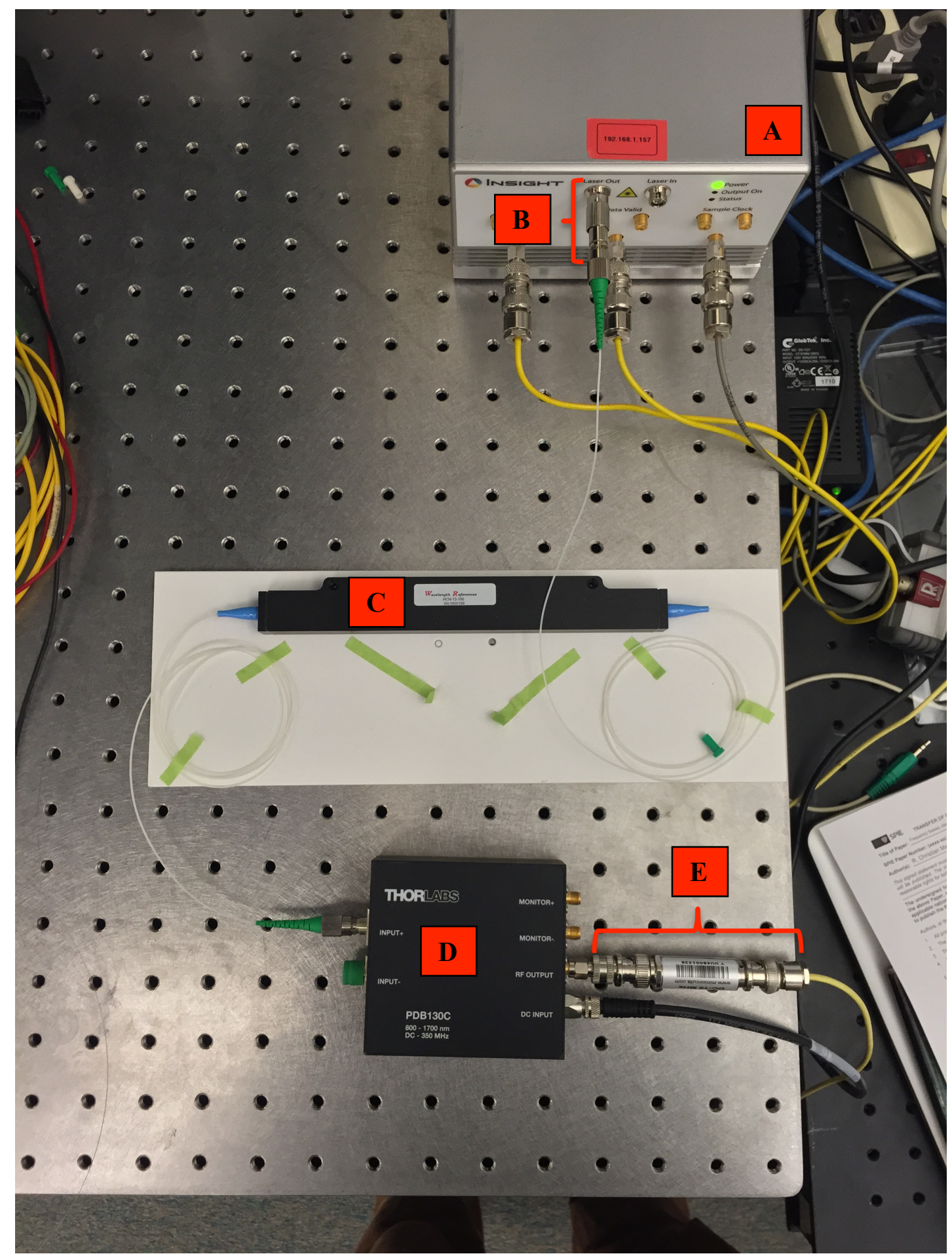

Figure 4-3 - The main components in the test setup shown in figure 4-1. The laser control software (figure 4-2) controls the laser system (A) through a computer and Ethernet connection. The laser output is coupled with a fiber attenuator (B) before going through reference device $2(\mathrm{C})$, a gas reference cell. The output of the reference cell is coupled onto the amplified photo detector (D) who's RF output is coupled into a low pass filter (E). 


\begin{tabular}{|cc|}
\hline Parameter & Value \\
\hline Minimum Wavelength & $1522.73 \mathrm{~nm}$ \\
\hline Maximum Wavelength & $1563.7 \mathrm{~mm}$ \\
\hline Output Power & $3 \mathrm{~mW}$ \\
\hline User Clock Rate & $400 \mathrm{MHz}$ \\
\hline Sweep Rate & $8.22 \mathrm{KHz}$ \\
\hline Power Profile & Flat \\
\hline Optical Frequency Step & $104 \mathrm{MHz}$ per $2.5 \mathrm{~ns}$ \\
\hline Optical Wavelength Step & $0.833 \mathrm{pm}$ per $2.5 \mathrm{~ns}$ \\
\hline
\end{tabular}

Table 1 - Typical VT-DBR laser parameters for jitter characterization

Once the user initiates a laser sweep via software, the laser's optical signal passes through a $3 \mathrm{~dB}$ optical attenuator to ensure that the amplified photo detector does not saturate. The optical signal then propagates through an optical fiber to a device under test (DUT 1/2/3) which modulates the optical signal. The devices under test are a gas reference cell, a 3-cavity $100 \mathrm{GHz}$ solid etalon, and an athermal fiber bragg grating centered at $1548.2 \mathrm{~nm}$. Each of these devices converts optical frequency fluctuations into measurable amplitude-time variations. These devices are further described below.

The signal is then coupled into a Thorlabs PDB130C indium gallium arsenide PIN high-speed balanced photo detector [11]. Only a single input is used for the purposes of this characterization. This photo detector is responsive from $\mathrm{DC}-350 \mathrm{MHz}$ making it suitable for recording high-speed VT-DBR sweeps.

Features of interest and to be recorded in this test setup occur at a measurement bandwidth of no more than $10 \mathrm{MHz}$. Since the amplified photo detector is responsive up to $350 \mathrm{MHz}$, broadband noise is also amplified and introduced into the RF output signal of the photo detector. 

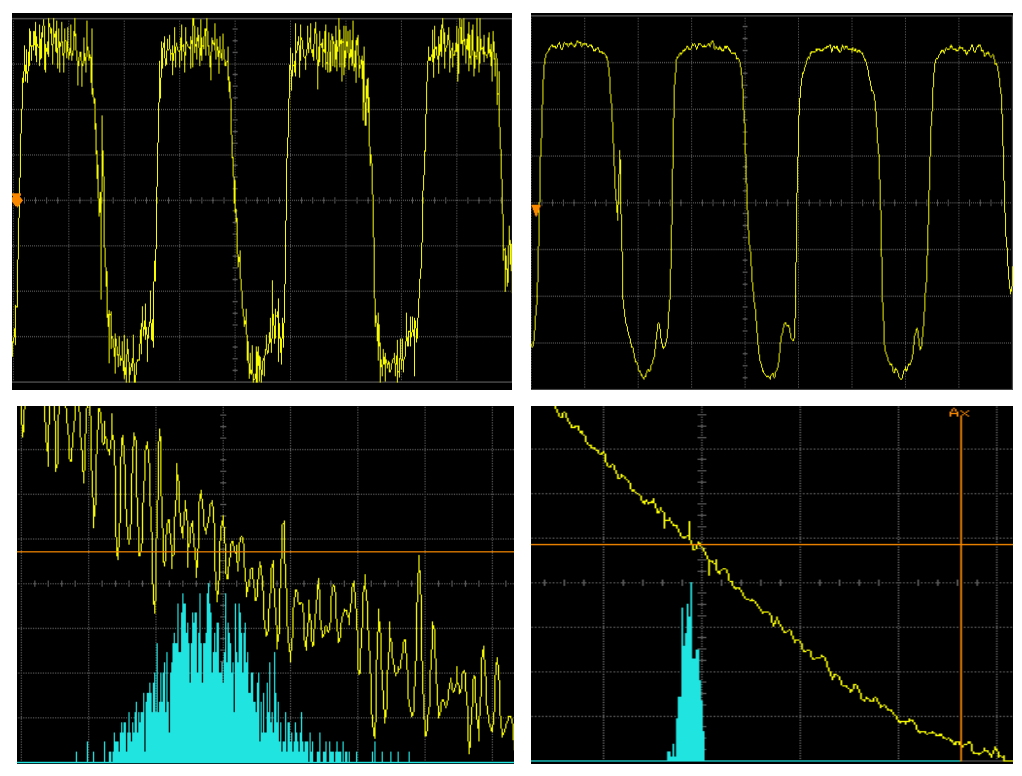

Figure 4-4 - Output signal of amplified photo detector with (right) and without (left) low-pass filter. The periodic waveforms on top represent periods of the etalon while the two captures on bottom represent histograms of two edge transitions. The peak-to-peak deviation of the unfiltered histogram (bottom left) is approximately $100 \mathrm{~ns}$ while the filtered histogram (bottom right) is only about $8 \mathrm{~ns}$.

A Mini-Circuits BLP-15+ (15542) analog low-pass filter with a $15 \mathrm{MHz}$ cutoff frequency is placed after the detector to remove this unnecessary noise amplified by the detector. It is interesting to note that without this filter, significant noise adds to the signal and causes peak-to-peak jitter to increase. Any filter also induces a time delay in the signal which is important to record for aligning signals in post-processing.

Jitter measurements were taken primarily on the Keysight 54845A Infiniium Oscilloscope. This scope provides 4 input channels to view all laser signals simultaneously (start sweep, data valid, sample clock, output signal from photo detector), as shown in figure 4-2, at a sample rate of up to $8 \mathrm{GSa} / \mathrm{s}$. This scope also features histogram functionality making it optimal for jitter measurements [12].

Aside from histogram statistics from the oscilloscope, the National Instruments PCI5105 digitizer (DAQ) was used to acquire jitter data. While a slower data acquisition 


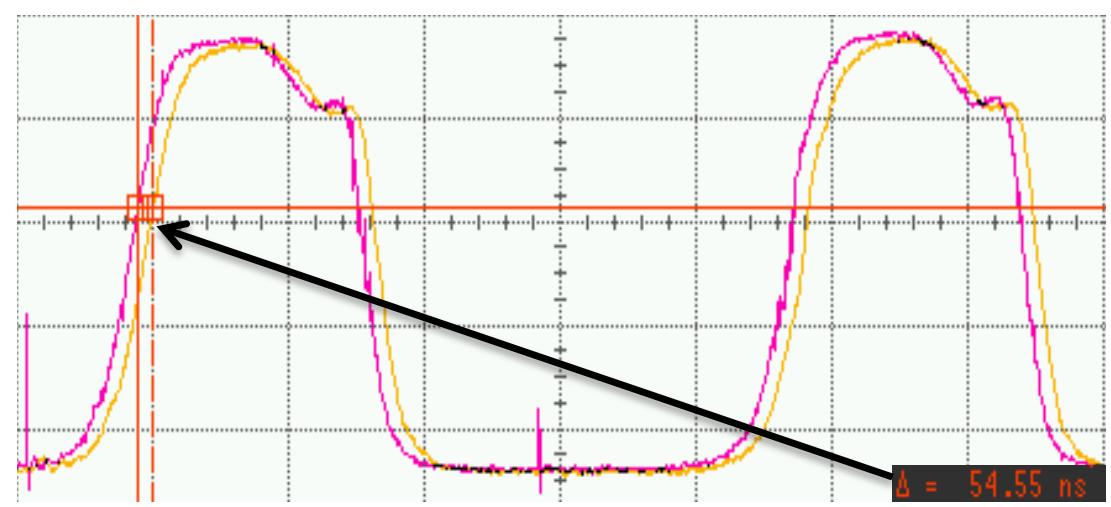

Figure 4-5 - Signal-to-noise ratio (SNR) ration improvement using a $15 \mathrm{MHz}$ low pass filter after the ThorLabs photo detector. The phase-leading (pink) signal is the raw output from the amplified photo detector. This photo detector amplifies any optical input signal and noise up to $350 \mathrm{MHz}$ while the signal of interest contains features in a measurement bandwidth of no more than $10 \mathrm{MHz}$. A low-pass filter is applied to the noisy signal to obtain the phase-lagging (yellow) signal, delayed by approximately $55 \mathrm{~ns}$ due to the filter group delay

rate of $50 \mathrm{MHz}$ must be used for this method, digital finite impulse response (FIR)

filtering and Matlab scripts allow for a unique analysis of jitter.

The first device under test used to interrogate optical frequency jitter is a molecular absorption based wavelength reference or gas absorption cell [13], shown in figure 4-5 below. A gas cell is a simple device with a single input and output containing a highly pure gas. The absorption peaks (observed as minima in the transmission spectrum) in the spectrum result from the fundamental vibrational and rotational energy levels of

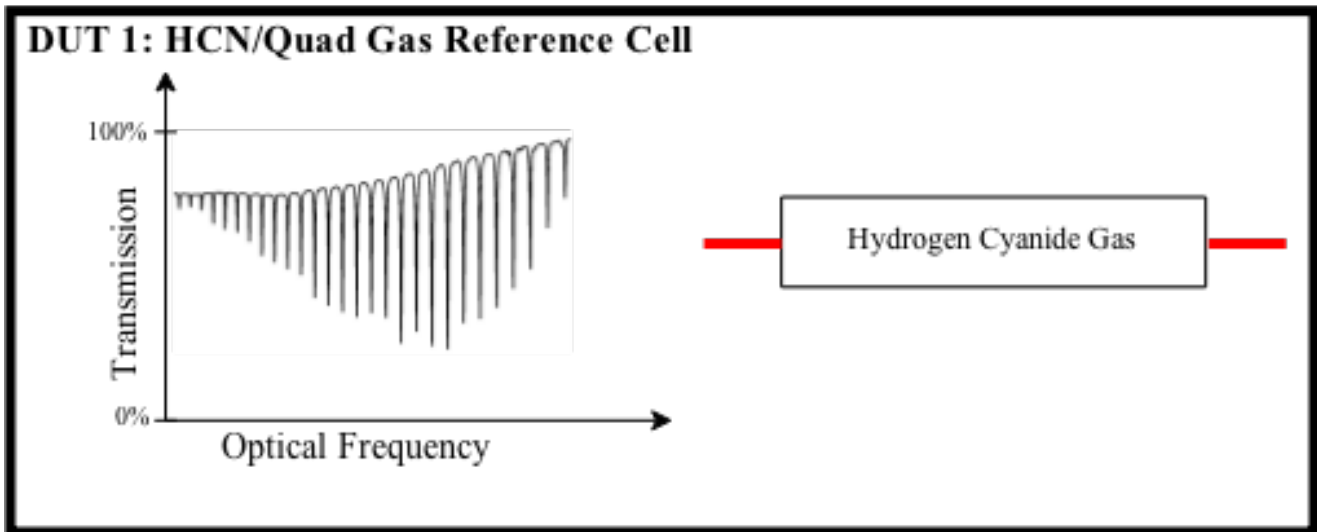

Figure 4-6 - Device under test 1 is a gas reference cell. The Hydrogen Cyanide cell is used in characterizing jitter. 
the gas molecules in the cell. If a photon with energy $E$, based on its wavelength, $\lambda$, the speed of light, c, and Planck's constant, h:

$$
E=\frac{h c}{\lambda}
$$

interacts with a molecule that has a vibrational or rotational energy level equal to $E$, the photon is absorbed by the molecule, causing it preventing its transmission. Gas cells are

\begin{tabular}{|cccc|}
\hline Model & Wavelength Range & Gas & Absorption Features \\
\hline WA-1530-1560 & $\mathbf{1 5 2 8 - 1 5 6 2 ~} \mathbf{~ n m}$ & $\mathbf{H}^{\mathbf{1 3}} \mathbf{C N}$ & $\mathbf{5 1}$ \\
\hline WA-900-1638 & $800-1638 \mathrm{~nm}$ & ${ }^{12} \mathrm{C}_{2} \mathrm{H}_{2},{ }^{12} \mathrm{CO},{ }^{13} \mathrm{CO}, \mathrm{H}_{2} \mathrm{O}$ & 28 \\
\hline
\end{tabular}

Table 2 - Gas cells used in this project. The WA-1530-1560 is used for jitter characterization due to its high number of absorption features in the range of a VT-DBR sweep.

beneficial because they are very stable over time, temperature, pressure, and strain.

Of the two gas cells used in this project, shown in table 2, jitter measurements were undertaken using the WA-1530-1560 Hydrogen Cyanide gas cell. This cell contains 51 absorption features over the range a typical VT-DBR sweep, while the WA-900-1638 Quad cell contains only 28 features across this same range. Absorption lines are accurate to $0.3 \mathrm{pm}$ with a temperature coefficient of $0.01 \mathrm{pm}$ per degree Celsius [14].
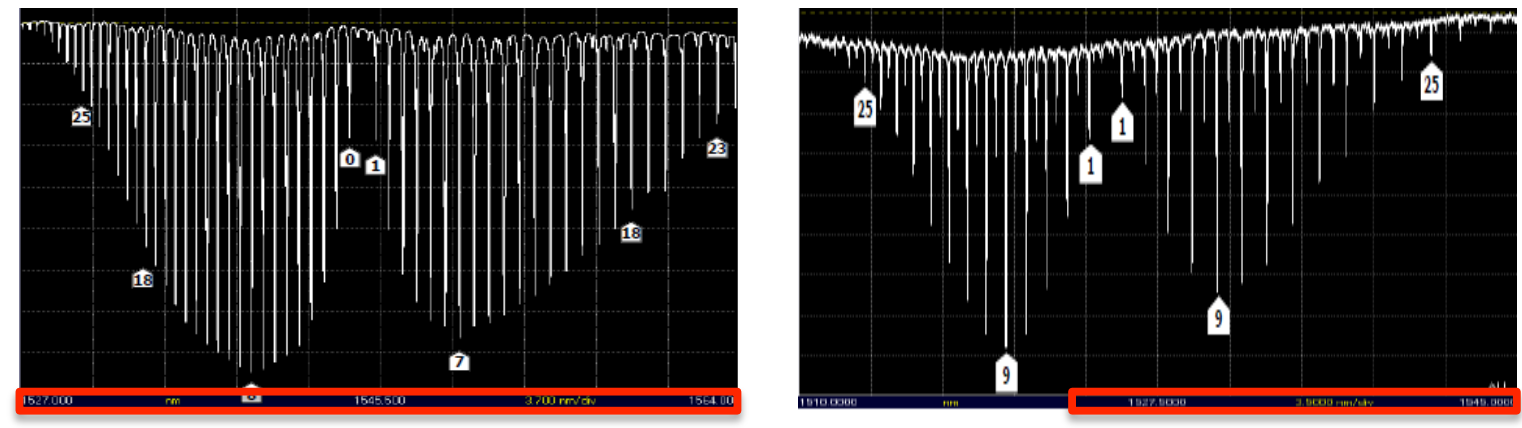

Figure 4-7 - Transmission spectra of WA-1530-1560 Hydrogen Cyanide cell (left) and WA-900-1638 Quad cell (right) and the range of a VT-DBR sweep is mapped on the horizontal axis in red [15]. 


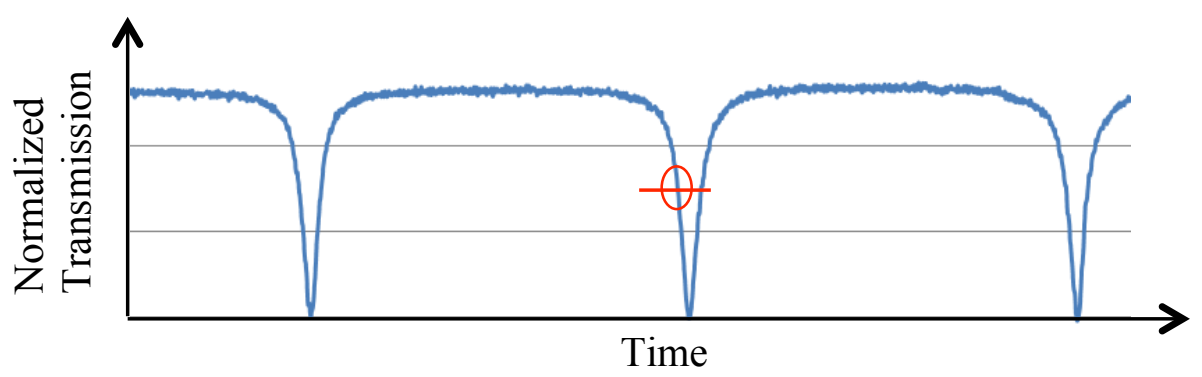

Figure 4-8 - Threshold location on a gas cell absorption line is at the steepest rate of change, generally near full width half maximum (FWHM). A marker is placed on the vertical location at which the falling edge of this etalon pass-to-stop band transitiont is measured.

In order to measure jitter using a gas cell, we zoom in on a single absorption feature. We then target a rising or falling edge of the absorption line and place a marker at the half maximum value, as shown in figure 4-8.

Based on the wavelength accuracy of the gas cell absorption lines, we can infer that any shift or jitter in time of this sharp rising or falling edge (any of the sharp rising or falling edges shown in figure 4-8) value can be understood to be a variation or jitter in optical frequency or wavelength - because the absorption feature represents a highly accurate and stable wavelength marker. A typical optical frequency step is on the order of $\Delta \mathrm{F}=104 \mathrm{MHz}$ per $2.5 \mathrm{~ns}$ following a self-calibration which corresponds to a wavelength step of $\Delta \Lambda=0.833 \mathrm{pm}$ per $2.5 \mathrm{~ns}$. Thus, we can easily compute the effective peak-topeak optical frequency and wavelength jitter based on the peak-to-peak time deviation of the maximum slope location of an absorption line.

Along with peak-to-peak measurements, we are also interested in the standard deviation or RMS jitter to gain a better understanding of how jitter is distributed. A peakto-peak value may often be large due to outliers that can easily be discarded through an RMS value. 


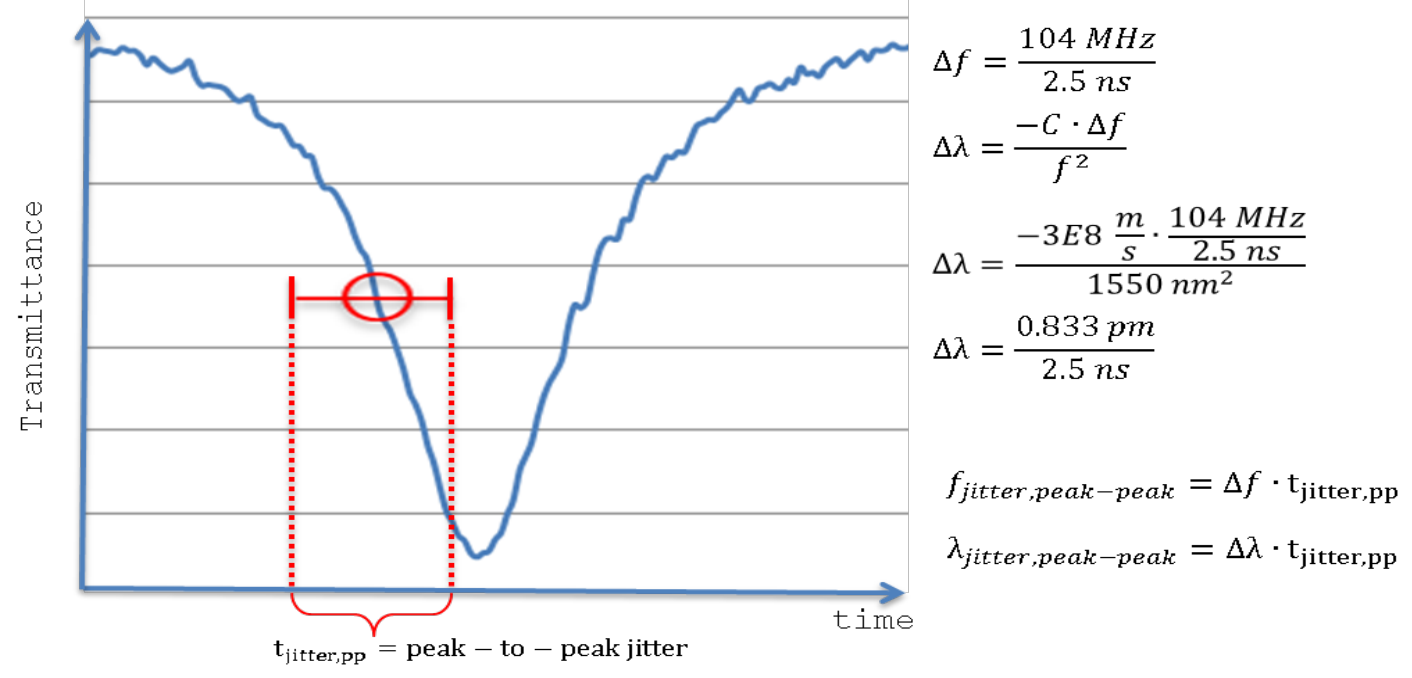

Figure 4-9 - Measuring peak-to-peak jitter is a matter of finding a well-defined slope on the output of the laser. The peak-to-peak time deviation of this point on the absorption spectrum is used to compute optical frequency and wavelength jitter.

The Keysight Technologies Infinium 54845B oscilloscope enables simple and accurate measurements of statistical information. By selecting an appropriate y-threshold value and a time window around a location where the waveform crosses this threshold, the scope begins to collect statistics about the y-crossing location in time. A typical ythreshold such as the half-maximum of a rising or falling edge of an absorption feature is generally chosen.

The first 3 devices under test use this same procedure to measure jitter in time and calculate the effective wavelength or optical frequency jitter. Since the sweep of the VTDBR should be very linear with respect to time, at any instant in time, a particular wavelength should emanate from the source. Since the absorption lines in a gas cell are highly stable with less than 0.3 pm deviation [14], we infer that any variations in location of absorption lines imply a corresponding variation in optical frequency and wavelength of the source. 

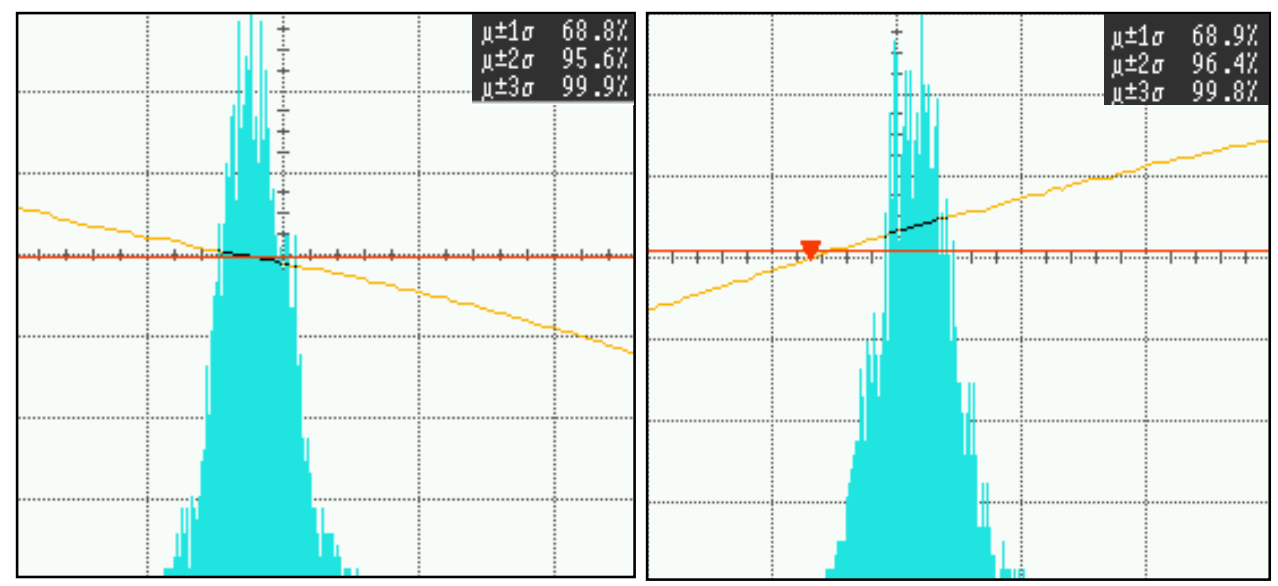

Figure 4-11 - Two histograms showing the Gaussian nature of jitter measured in all methods.

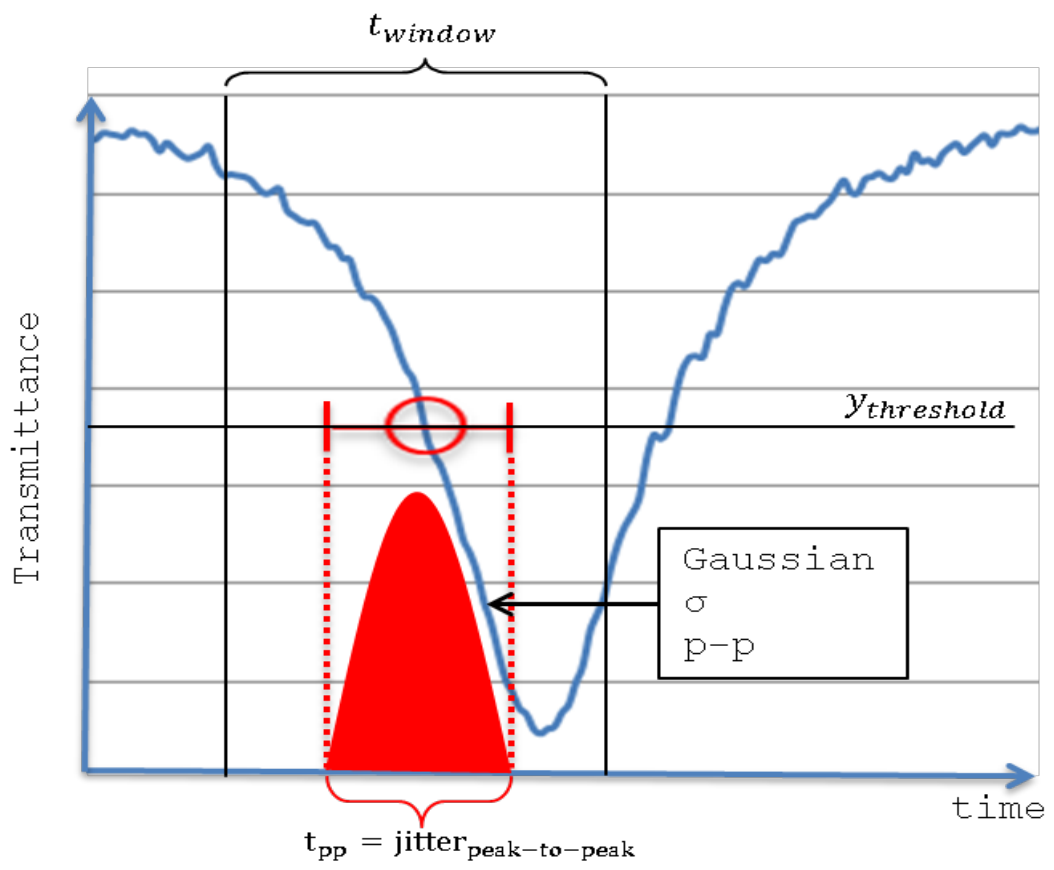

Figure 4-10 - The histogram created by the oscilloscope is nearly Gaussian. The standard deviation, peak-to-peak value, as well as number of tabulated results are reported. 


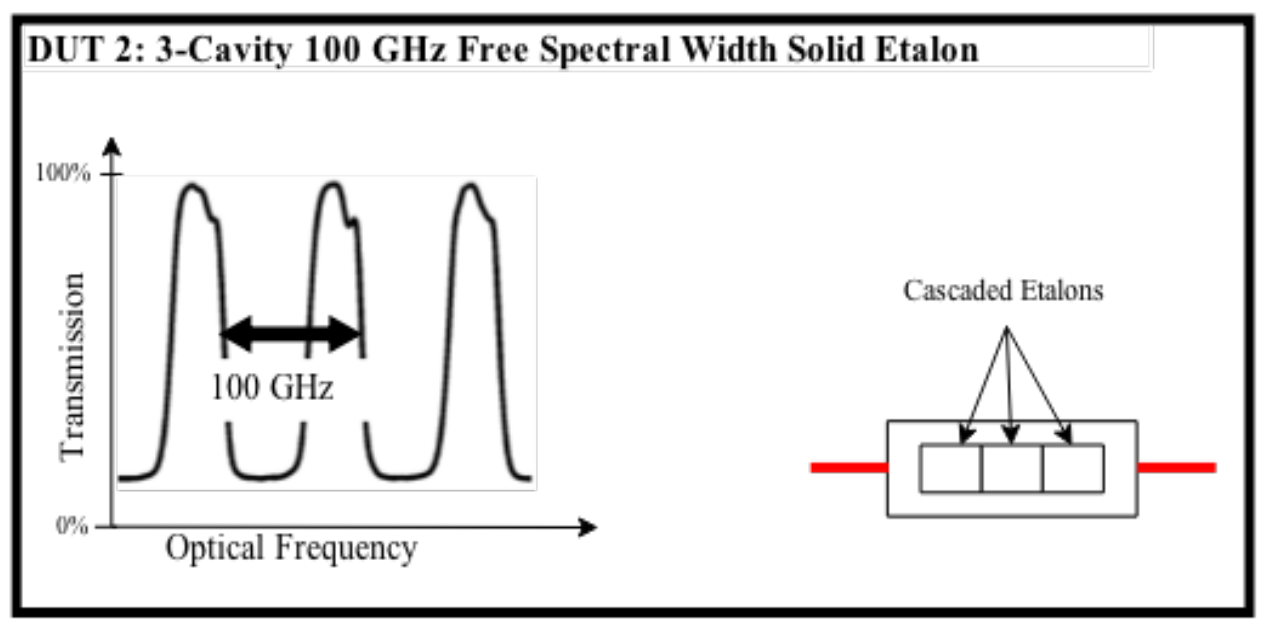

Figure 4-12 - DUT 2 is a 3-Cavity $100 \mathrm{GHz}$ Etalon with the transmission characteristic shown.

The second device under test is a 3-cavity $100 \mathrm{GHz}$ solid etalon. An etalon is a simple Fabry- Pérot interferometer composed of a single plate with two parallel reflecting surfaces. Light which enters this interferometer is reflected internally and interferes with itself constructively or deconstructively depending on the wavelength or optical frequency. The unique device used for this experiment contains 3 cascaded etalons, creating very sharp edge pass - band to stop-band and stop-band to

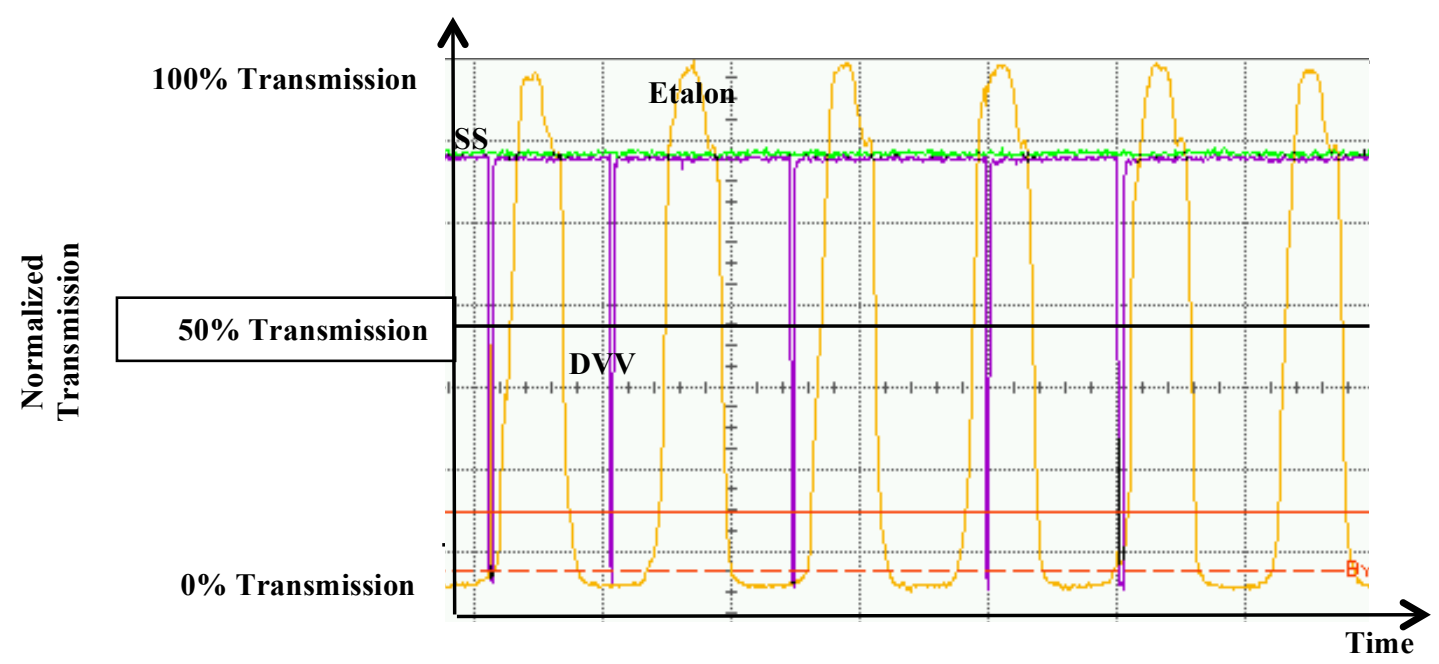

Figure 4-13 - Oscilloscope capture showing the etalon output with the VT-DBR sweeping as an input. Note that peaks of the etalon waveform are separated by $100 \mathrm{GHz}$. The data valid vector (DVV) and start sweep (SS) triggers are also shown. 
pass-band transitions in the output transmission spectrum. It is also a solid device making it resistant to vibrations or external environmental changes. As shown in figure 4-12, the free spectral range of this etalon is measured from edge-to-edge or from peak-to-peak to be $100 \mathrm{GHz}$.

This solution was chosen over of a standard Mach-Zehnder interferometer (MZI) which was initially explored as a device under test. The unique solid construction of this etalon provides increased stability against mechanical strain and vibrations as well as temperature fluctuations, to which a standard MZI is very sensitive. A standard MZI has temperature sensitivity on the order of $10 \mathrm{pm} /$ degree Celsius. The concatenated 3 cavities create sharper, square-wave style edges as opposed to the sinusoidal output of a MachZehnder interferometer. These sharper edges also provide a better measurement apparatus for studying jitter.

By using the histogram function on the sharp pass-band-to-stop-band and stopband-to-pass-band transitions of the etalon, another method of measuring VT-DBR jitter exists. Since these sharp transitions occur at a repetitive wavelength or optical frequency, we can measure the $50 \%$ transmission location time of these transitions to calculate RMS and peak-to-peak optical frequency and wavelength jitter.

By placing a y-threshold value at approximately 50\% transmission as shown in figure 4-13 and placing a time window around a single transition edge, a measurement for jitter may be obtained using the histogram feature of the scope. 

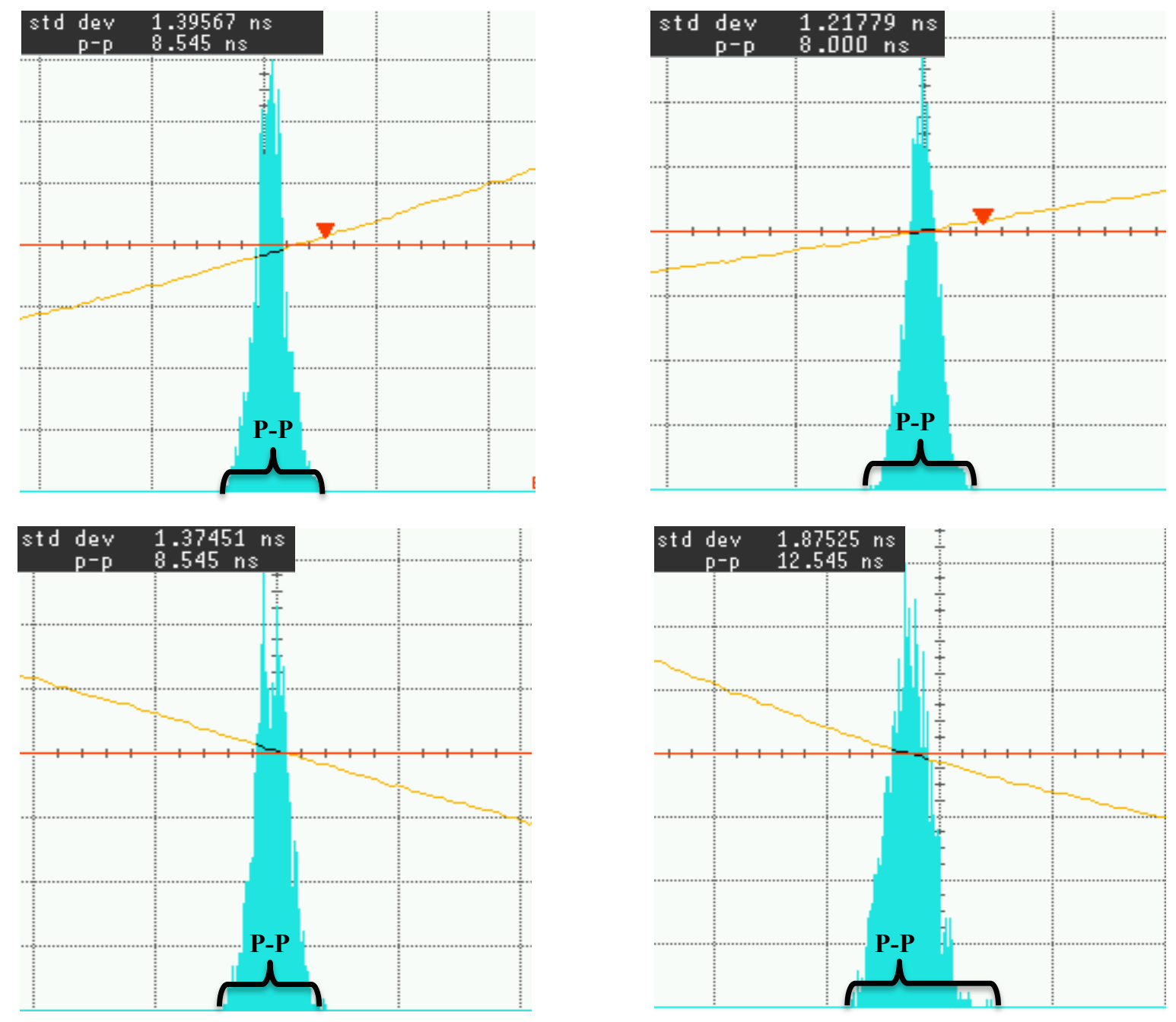

Figure 4-14 - Two histograms of a rising etalon edge (top) and two histograms of a falling etalon edge (botton. The etalon signal is shown in yellow and the histogram appears in turquoise. Note the Gaussian nature of the histograms. Each histogram has $1000+$ bins taken over 10 seconds. 


\section{DUT 3: Athermal Fiber Bragg Grating at $1548.1851 \mathrm{~nm}$}
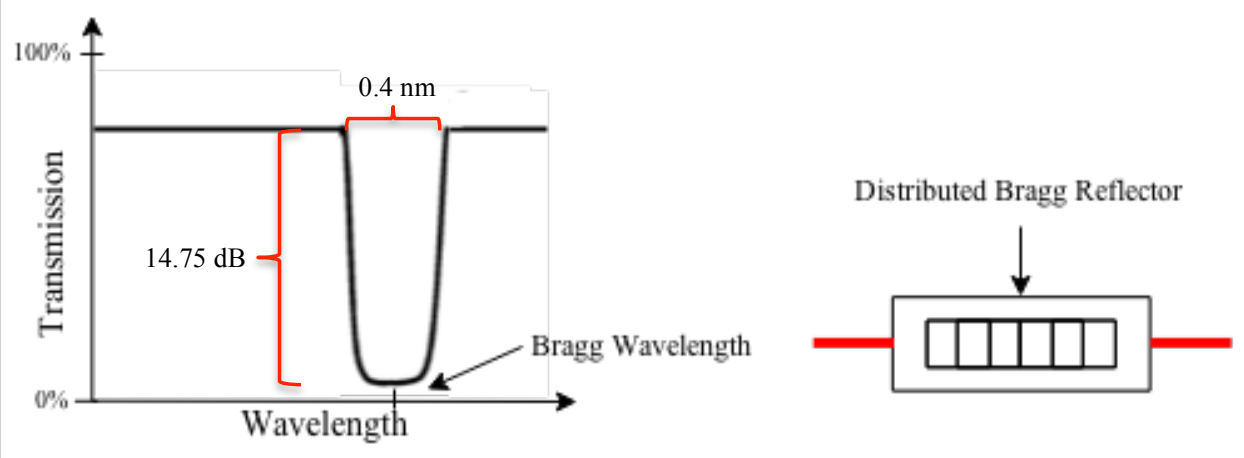

Figure 4-15 - DUT 3 is a fiber Bragg grating which is temperature-compensated (athermal) and encapsulated for protection from the environment. This FBG has a maximum attenuation of $14.75 \mathrm{~dB}$ and FWHM of $0.4 \mathrm{~nm}$.

The third device under test is an athermal fiber Bragg grating at $1548.1851 \mathrm{~nm}$ from CiDRA. This grating is temperature-compensated (athermal) and encapsulated to make it robust against external and environmental vibrations or temperature fluctuations. The grating measures approximately $0.4 \mathrm{~nm}$ FWHM with a maximum attenuation of $14.75 \mathrm{~dB}$ at $1548.1851 \mathrm{~nm}$.

To measure the optical frequency or wavelength jitter using an FBG, the two edges (falling and rising) surrounding the Bragg wavelength of the grating are measured at half maximum transmission. As with the gas cell and etalon, we place a y-threshold at half maximum of the FBG output and monitor the rapid fluctuations in time of the ythreshold crossing. As with the previous two acquisitions, the low-pass filter is a crucial component in reducing excess noise in the signal of interest.

The final method of optical frequency and wavelength jitter interrogation utilizes the gas absorption cell, but through a process different from the previous 3 . In this method, the NI PCI5105 digitizer card was used to acquire several hundred sweeps 
through the gas reference cell in rapid succession. Absorption minima were identified (shown as triangle markers in figure 4-16) in the raw data. The absorption lines were approximated as a polynomial and a mathematical curve fit was undertaken to the minima from each sweep. Depending on the strength of the absorption feature, up to 11 data points surrounding a minimum were used to fit an absorption feature.

This method was realized because the digitizer is limited to a $60 \mathrm{MS} / \mathrm{s}$ sampling rate. Furthermore, in order to synchronize properly with the VT-DBR laser, an integer divisor of the VT-DBR sample clock of $400 \mathrm{MHz}$ must be used. Thus, the maximum acquisition rate of the digitizer is $50 \mathrm{MS} / \mathrm{s}$ meaning that although the VT-DBR changes optical frequency or wavelength every $2.5 \mathrm{~ns}\left(\frac{1}{400 \mathrm{MHz}}\right)$, the digitizer will only acquire a laser data point every $20 \mathrm{~ns}\left(\frac{1}{50 \mathrm{MHz}}\right)$, or every 8 samples of the laser output. Due to the intrinsic polynomial shape of the gas absorption features, we argue that approximating the shape of an absorption feature may be done by fitting a simple, second-order

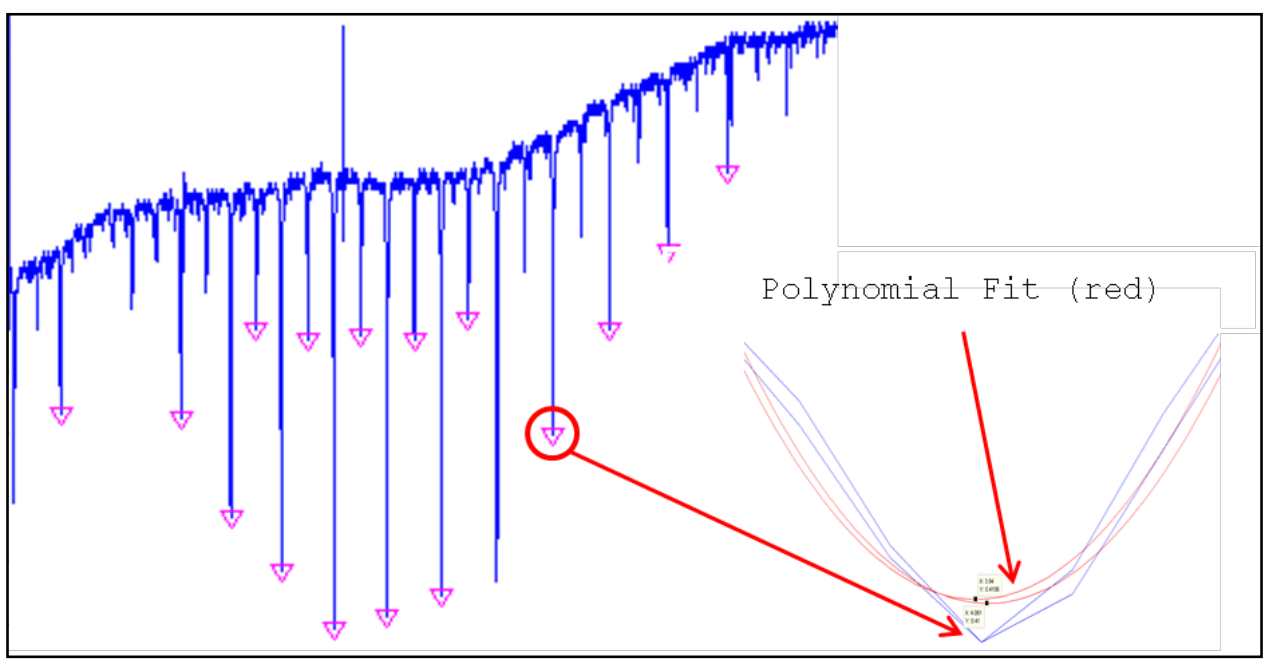

Figure 4-16 - Full spectrum of gas cell (left, blue) and a zoomed in view of a single absorption peak. The blue and red curves on the right show two absorption peaks superimposed with their polynomial fit curves in red. 
polynomial to the points surrounding an absorption feature [13]. This allows us to resolve a more accurate location for the absorption peak which we are intrinsically limited on.

To achieve this polynomial fit processing, a Matlab script was written. While this method is the most subjective to errors and uncertainties, we report optical frequency or wavelength jitter on the same magnitude as with other methods, exhibiting a similar Gaussian or normal distribution.

In a final jitter measurement, we observe the effect of placing a long length of fiber before measuring the optical frequency jitter using a DUT. In real fiber sensing applications, kilometers or even 100s of kilometers of fiber may be required interface with a sensor that is far away. The main purpose behind this test is to more accurately simulate jitter that may exist when using the VT-DBR as a source for fiber sensing applications.

A $25.3 \mathrm{~km}$ SMF-LS(TM) fiber was inserted into the test system before the DUT the 3-cavity solid etalon. The reflection from the etalon was monitored instead of the transmission to more accurately simulate optical signals that would be obtained from an actual sensor.

\subsection{Jitter of the VT-DBR}

Based on histograms of all 51 absorption features in the Hydrogen Cyanide gas cell, average RMS jitter of $0.45 \mathrm{pm}$ with a maximum of $1.15 \mathrm{pm}$ is reported. Each histogram comprises no less than 1000 y-crossing time locations (bins). Figure 4-17 shows the results of RMS wavelength jitter.

Statistical data of time jitter from 106 etalon edges across a single sweep, yields RMS wavelength jitter no worse than $1.57 \mathrm{pm}$ with an average of $0.5 \mathrm{pm}$ as shown in 
figure 4-17. Over 3 measurement trials, where the time jitter of each etalon rising or falling edge is measured each time, results show wavelength jitter less than $1 \mathrm{pm}$ for the first half of the sweep, with only a few outliers above $1 \mathrm{pm}$ in the last $15 \mathrm{pm}$ of each sweep.

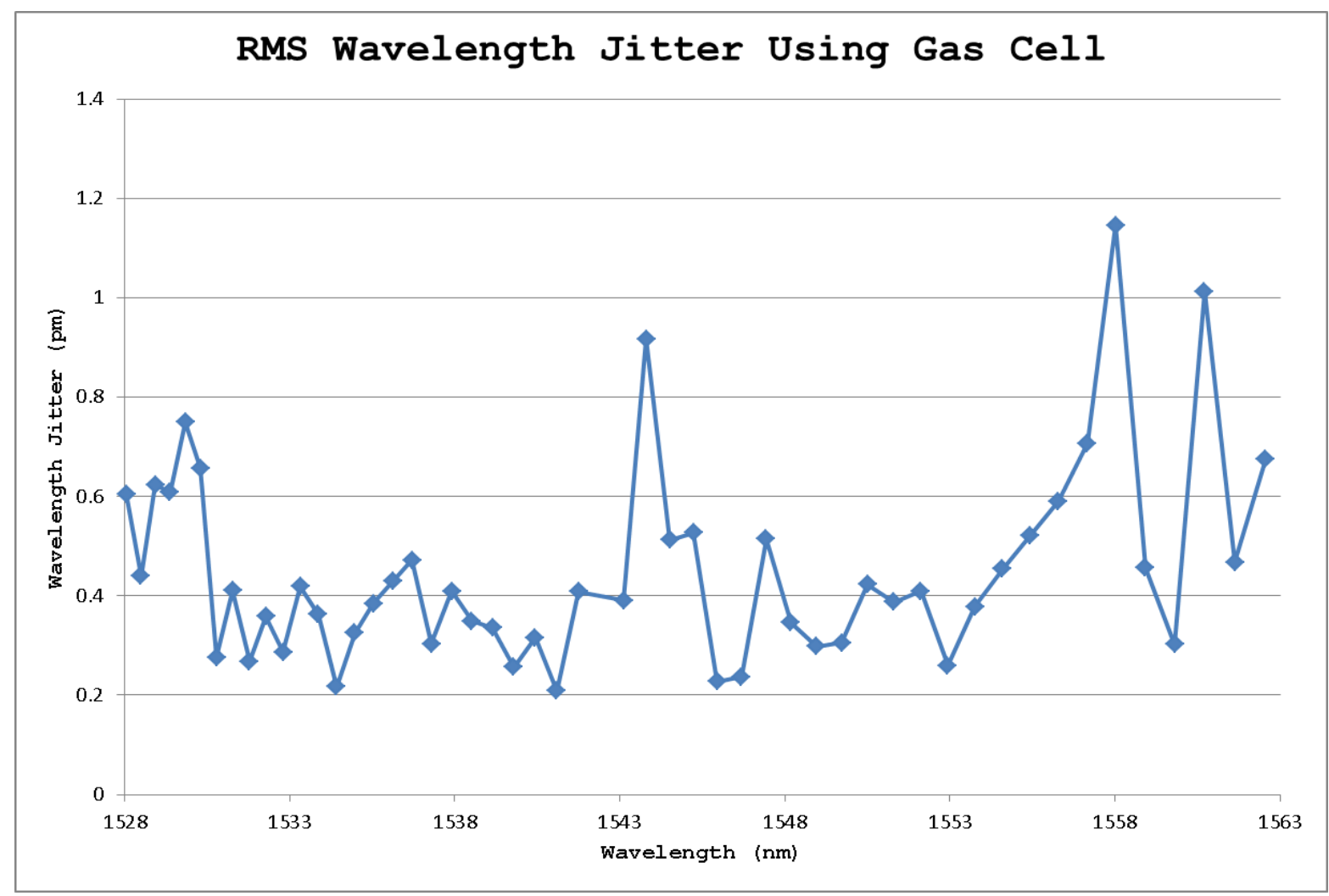

Figure 4-17 - Optical wavelength jitter of the VT-DBR as measured through the Hydrogen Cyanide gas cell with 51 absorption features across nearly the entire sweep range of the laser. The laser is swept at $4 \mathrm{~mW}$ and an $8.2 \mathrm{kHz}$ sweep repetition rate. 


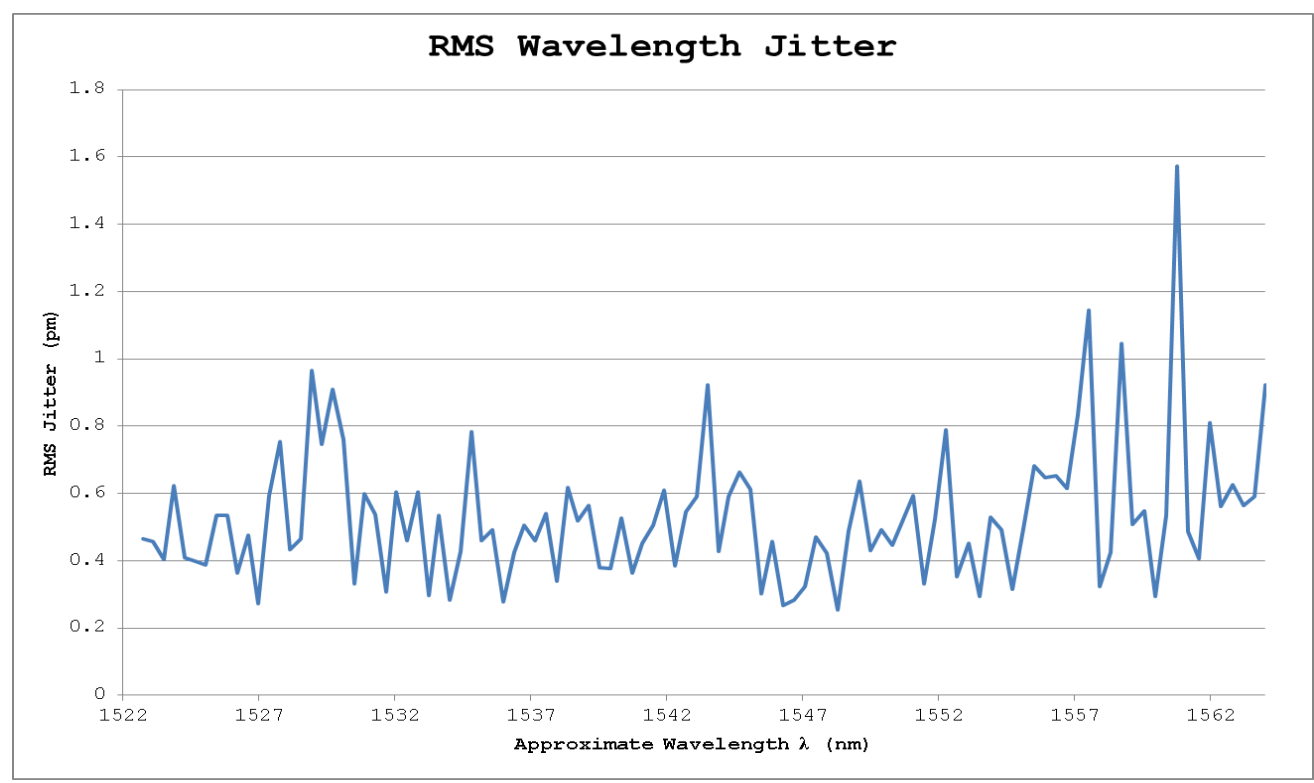

Figure 4-18 - RMS wavelength jitter measured using the etalon. Jitter values are aligned with their approximate wavelengths, calculated based on the $100 \mathrm{GHz}$ free spectral range of the etalon.

Measuring jitter over 5 trials using the athermal fiber Bragg grating, an optical wavelength jitter on the order of 0.42 pm RMS is observed. Results are shown in table 3 below.

\begin{tabular}{|c|c|c|c|}
\hline Trial & Edge & RMS (pm) & RMS (MHz) \\
\hline 1 & Rising & 0.26 & 31.95 \\
\hline 1 & Falling & 0.42 & 52.82 \\
\hline 2 & Rising & 0.37 & 45.91 \\
\hline 2 & Falling & 0.53 & 66.91 \\
\hline 3 & Rising & 0.35 & 44.07 \\
\hline 3 & Falling & 0.59 & 73.46 \\
\hline 4 & Rising & 0.35 & 44.22 \\
\hline 4 & Falling & 0.49 & 61.07 \\
\hline 5 & Rising & 0.35 & 43.95 \\
\hline 5 & Falling & 0.48 & 60.26 \\
\hline
\end{tabular}

Table 3 - Summary of jitter measurements conducted using an athermal fiber Bragg grating. The rising and falling edge of the reflected Bragg wavelength are monitored and recorded. Results verify findings from previous measurements using other DUTs. 
Last, the method of polynomial fitting to determine jitter results in a peak RMS wavelength jitter of $1.35 \mathrm{pm}$ and an average RMS wavelength jitter of $1.11 \mathrm{pm}$. These values correspond to a maximum of $169 \mathrm{MHz}$ optical frequency jitter and an average of $139 \mathrm{MHz}$. Again, these results verify findings from previously described methods and confirm the feasibility of polynomial fitting gas absorption lines.

In a final examination of jitter, a $25 \mathrm{~km}$ length of fiber is inserted into the optical signal path to observe the effect of long propagation distances on jitter. The etalon is used as the DUT. A maximum peak-to-peak jitter of $8.71 \mathrm{pm}$ and max RMS jitter of $1.33 \mathrm{pm}$ is observed. These values are similar to those obtained from previous with no fiber in the system.

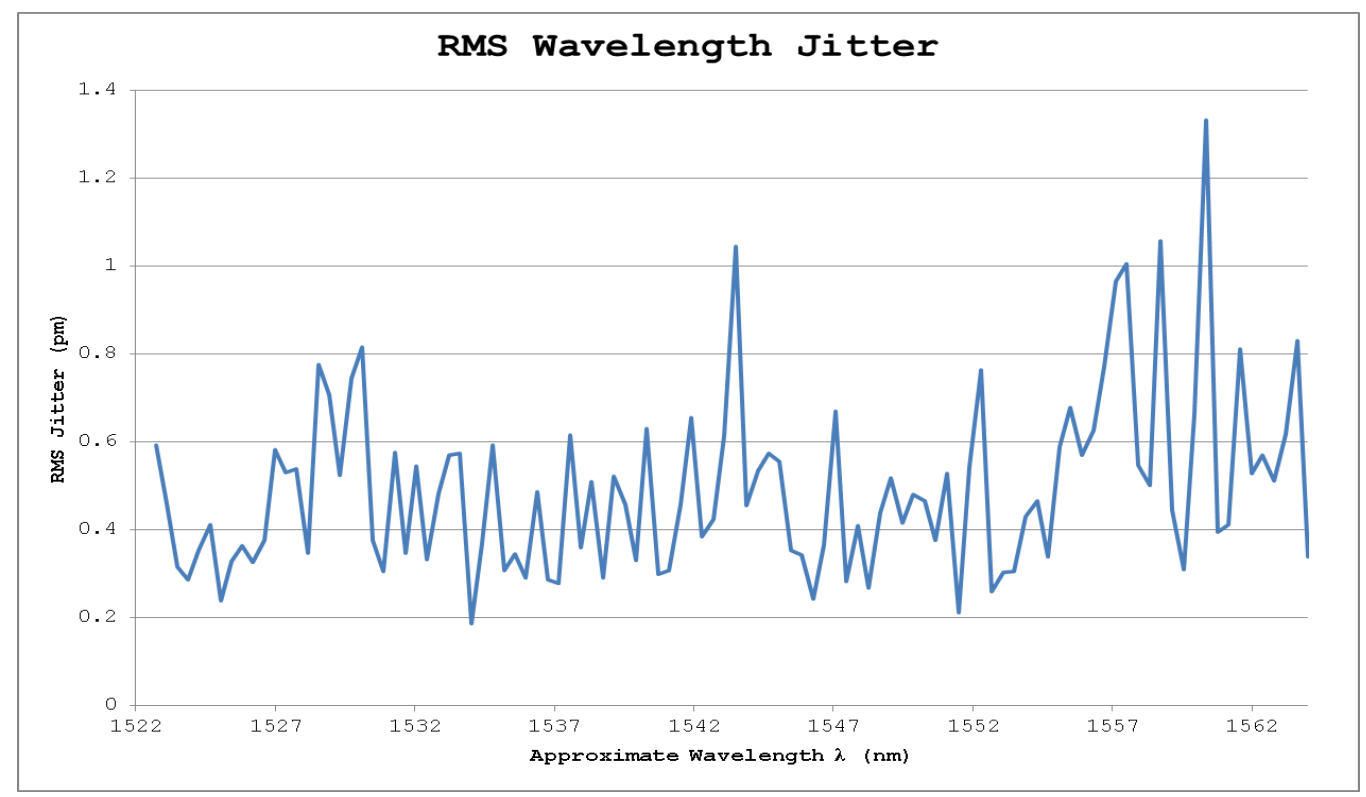

Figure 4-19 - RMS Wavelength jitter measured using the solid etalon DUT and $25 \mathrm{~km}$ of fiber in the optical signal pathway. Results are on the same order of magnitude as other measurements with very little fiber length. 


\subsection{Jitter of a 2nd Generation VT-DBR}

To improve upon multiple aspects of the laser system, newer modules have been developed. These upgraded modules include upgraded drive circuitry and improved noise reduction mechanisms. Characterizing a newer module gives insight on the advantages and disadvantages of implemented modifications. This also informs on the current state of the laser system rather than outdated systems with worse performance.

The same test setup and procedure is used for measuring jitter in the new module. Measurements are conducted using only the HCN gas reference cell due to availability of other DUTs at the time that a new module was acquired.

Results show improved jitter with an average of $0.35 \mathrm{pm}$. We note that there are no significant outliers or spikes in jitter as was observed in the previous unit.

We also observe a ramp nature of the plot in figure 4-20 which leads us to conclude that jitter may be dependent on laser drive currents. Laser drive currents exhibit

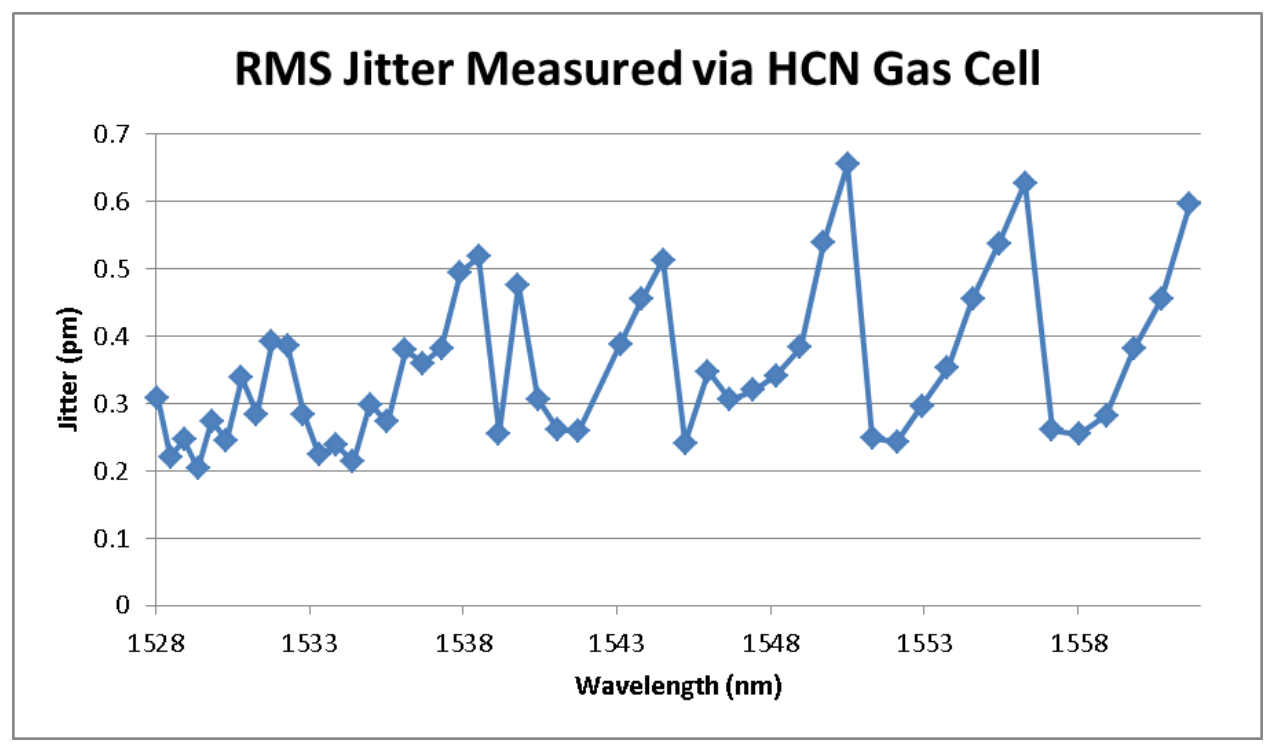

Figure 4-20 - The new 2nd generation VT-DBR laser system with improved drive circuitry and noise reduction mechanisms exhibits jitter on the same order as the previous generation, but slightly lower with less jitter "spikes" or outliers. The ramp nature of the jitter vs. wavelength plot is noted. 
a similar ramp-nature as wavelength is dynamically swept and are a likely contributor to jitter, so we conclude that jitter is intrinsically related to dynamic drive currents in the laser.

More specifically, we hypothesize that at higher drive currents, more jitter will be observed in the output wavelength of the laser as opposed to lower drive currents.

Another hypothesis is that the areas of higher jitter occur near supermode transitions within the laser. This could be further investigated and understood by viewing a tuning map for this VT-DBR model. 
4.4 Summary and Analysis of Jitter Results

\begin{tabular}{|cccc|}
\hline DUT & & Maximum & \\
& Average RMS & RMS & Peak-to-Peak \\
\hline Gas Absorption Cell & $0.45 \mathrm{pm}$ & $1.15 \mathrm{pm}$ & $23.1 \mathrm{pm}$ \\
\hline Solid Etalon & $0.5 \mathrm{pm}$ & $1.57 \mathrm{pm}$ & $8.81 \mathrm{pm}$ \\
\hline Fiber Bragg Grating & $0.42 \mathrm{pm}$ & $0.59 \mathrm{pm}$ & $9.18 \mathrm{pm}$ \\
\hline Gas Absorption Cell PolyFit & $1.11 \mathrm{pm}$ & $1.35 \mathrm{pm}$ & N/A \\
\hline 25 km SMF-LS Fiber & $0.59 \mathrm{pm}$ & $1.33 \mathrm{pm}$ & $8.71 \mathrm{pm}$ \\
\hline
\end{tabular}

Table 4 - Summary of jitter in picometers from the different methods of interrogation undertaken in this project for the first generation VT-DBR module. For each of these results, observation times of no more than 5 seconds were allowed.

Table 4 summarizes optical wavelength jitter of the first generation VT-DBR system as measured through several different devices under test and methods. Results from all different approaches find that wavelength jitter in the VT-DBR laser source is on the order of picometers and that longer propagation times do not necessarily amplify or accumulate jitter. Interrogating jitter in a new module yields similar values, with slight improvements to jitter measurements on the order of $0.35 \mathrm{pm}$ RMS.

Optical frequency or wavelength jitter, regardless of the method of interrogation, tends to a Gaussian or normal distribution with close adherence to the 68-95-99.7 percent rule. This rule states that $68 \%$ of values or hits lie within 1 standard deviation of the mean, 95\% lie within 2 standard deviations, and $99.7 \%$ lie within 3 standard deviations of the mean. While the values are never exactly $68 \%, 95 \%$, and $99.7 \%$, values are close to these percentages and have a shape that is clearly identifiable as Gaussian. 
Jitter of a Gaussian shape leads to several conclusions. First, we propose that intrinsic random noise underlies the mechanisms which cause wavelength jitter. Even if there are less stochastic, predictable processes contributing to jitter, a Gaussian distribution implies that random noise, to some degree, contributes.

Presently, underlying mechanisms of jitter are thought to be due to the complex electronic drive circuitry of the laser. Since this laser source is controlled directly by 5 currents, 3 of which are frequency modulated, we assert that if these drive currents are fluctuating in any way, this will induce jitter in the output wavelength. It may be possible to reduce the impact of these mechanisms slightly by reducing drive current fluctuations, but the Gaussian distribution hints a certain amount of random optical frequency jitter will always be present in the system.

The Gaussian shape also informs us that averaging of laser sweeps may be a viable and beneficial way to increase the accuracy and precision of this laser. The theoretical basis for sweep averaging is that a Gaussian distribution has a normal mean

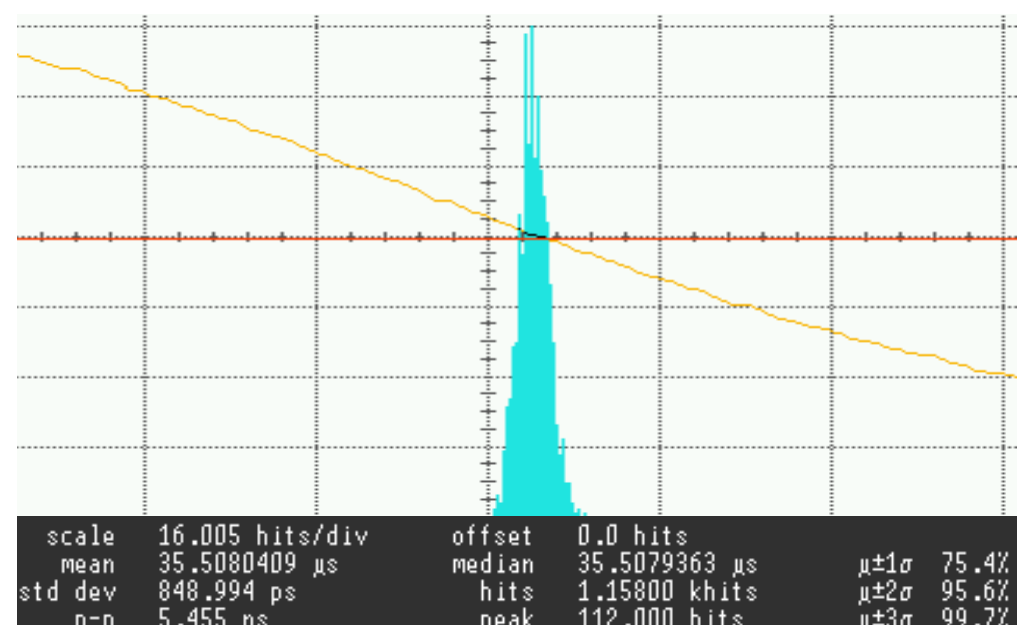

Figure 4-21 - A histogram of a sharp transition measuring intrinsic jitter of the VT-DBR system. Note the Gaussian nature of the distribution and the peak-to-peak value of 5.455 ns with an RMS of 0.849 ns. 
value that it tends towards - the average of the distribution. Over any significant number of sweeps (10 or more, for example), this jitter distribution may be observed and the average value calculated. Moreover, at sweep rates which may approach $1 \mathrm{MHz}$, averaging 100 sweeps only degrades the sweep repetition rate to $10 \mathrm{kHz}$, which is still much higher than other market solutions.

Implementing sweep averaging may reduce jitter by several orders of magnitude and will be proportional to the number of sweeps averaged.

For example, 1000 hits are recorded rapidly. Peak-to-peak jitter of $5.455 \mathrm{~ns}$ and RMS jitter of $0.849 \mathrm{~ns}$ is obtained. We record that $75.4 \%$ of the 1000 values lie within 1 standard deviation of the mean. Therefore, we disregard outliers and use the average value of the distribution as our recorded wavelength. Such a distribution implies an uncertainty of the RMS value instead of the peak-to-peak value. Ultimately, sweep averaging allows more accurate, precise wavelength measurements as opposed to using peak-to-peak wavelength jitter.

Wavelength jitter measurements also inform on the intrinsic linewidth of this laser as well. This result is a byproduct of the intended research, but provides insight into performance of the VT-DBR and helps verify current metrics. 

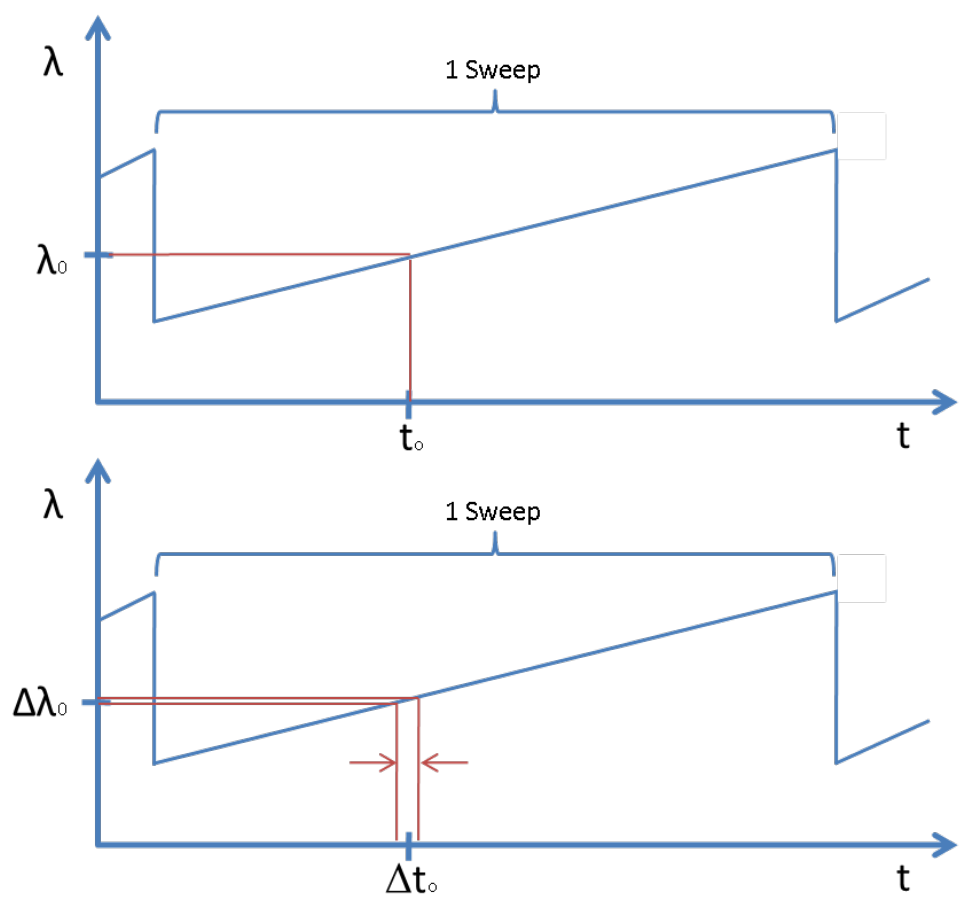

Figure 4-22 - An ideal linewidth is represented in the graph on top, where a wavelength $\lambda_{0}$ occurs at a single instant in time $t_{0}$. In reality, a wavelength $\lambda_{0}$ occurs over a small range of time $\Delta \mathrm{t}_{0}$ which represents the linewidth of the laser.

As with jitter measurements of swept laser sources, there is no universal or standardized way to measure linewidth or effective linewidth of a swept laser. We can, however, say that during a laser sweep, any particular wavelength that is within the range of the sweep should always occur at a well-defined, repeatable instant in time.

As we measure the particular instant in time that a wavelength should occur at, we actually measure a range of times over which this wavelength occurs. We know, however, that these ranges of times correspond to a single wavelength. Thus, the range of times measured (jitter) actually represents the width of the wavelength measured.

Insight Photonics reports that the linewidth of the VT-DBR is no better than 10 MHz. Reviewing the measured results from this section, we report an average linewidth between 50-70 MHz with minimum values just above $20 \mathrm{MHz}$. 
These values agree with the limiting $10 \mathrm{MHz}$ value provided by the VT-DBR manufacturer. We expect values on the same order of magnitude, but not necessarily close to $10 \mathrm{MHz}$ because our measurements are not taken with the purpose of measuring linewidth.

Regarding the polynomial fitting method of jitter interrogation, several sources of error are identified. First, gas cell absorption lines may appear polynomial in nature, but their true form is Doppler broadened Voigt profile which results from a collision broadened Lorentzian profile convolved with a Gaussian Doppler profile [13].

For preliminary work, approximating the Voigt profile as a second-order polynomial suffices, but it is important to acknowledge that this does introduce error into the calculations of jitter. Future work in polynomial fitting of absorption peaks should utilize other methods to approximate the complex profile of gas absorption cell lines.

With regard to fiber Bragg grating and other fiber optic sensing, the jitter measured in this project implies a limit on accuracy and precision. This limit depends on the sensor being implemented as well as the application, but we conclude that the VTDBR source in its current state is limited to resolving wavelengths with an error or uncertainty on the order of $0.5-1 \mathrm{pm}$. Ultimately, this uncertainty in wavelength leads to uncertainty in the measured parameter (i.e. temperature, strain, etc.). 


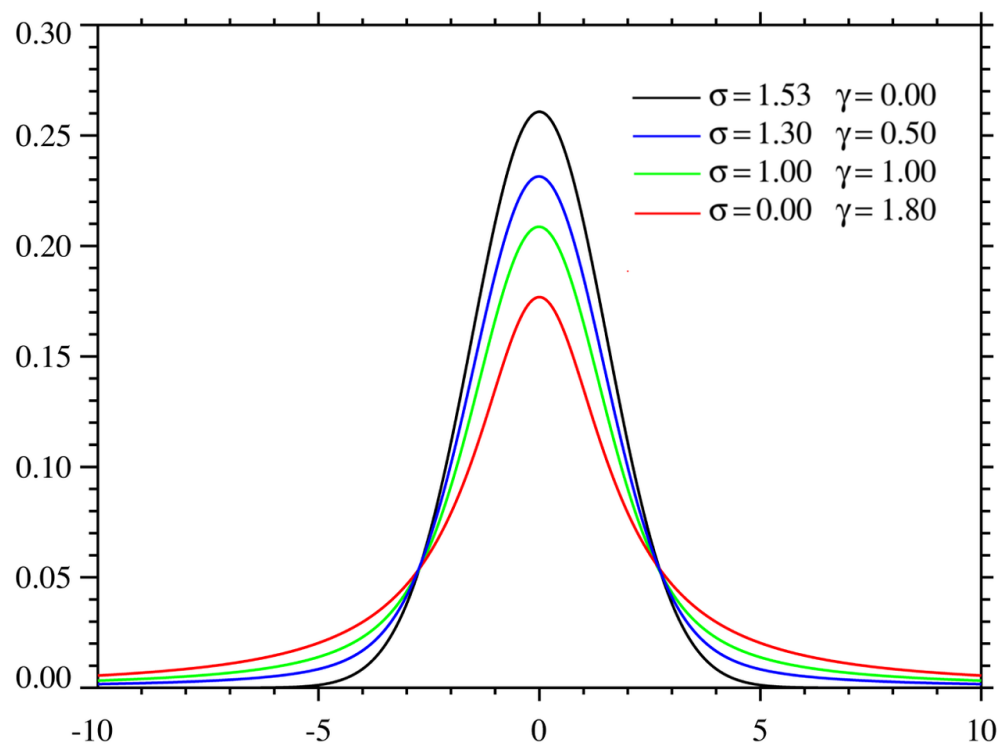

Figure 4-23 - The Voigt profile is a result of collisional broadening (described by a Lorentzian profile) and a Gaussian Doppler profile which appears somewhat polynomial [13].

Although SSOCT has already been demonstrated with the VT-DBR, the intrinsic jitter may ultimately limit the obtainable resolution of a scan. Especially in PhOCT, any jitter in the source corresponds to an optical phase jitter. This limits the resolution that a PhOCT scan can resolve.

Optical wavelength jitter in the VT-DBR appears on the order of picometers. This corresponds to an optical frequency jitter on the order of $100 \mathrm{MHz}$. These results do not have a significant impact on the success of the VT-DBR source as it is used currently. As applications become more demanding and higher resolutions are desired, however, intrinsic jitter may be the limiting factor.

A further investigation into the fundamental causes of jitter should be undertaken. Such a study may suggest a method to further reduce jitter uncertainty. Ideally, the VTDBR should be able to resolve sub-picometer or angstrom level resolution for extremely sensitive and precise fiber optic sensor and OCT applications. 


\section{WANDER CHARACTERIZATION}

Now that short-term, high-frequency variations in the VT-DBR sweep have been addressed and quantified, longer-term variations in the system are investigated. A method for calibrating a sweep using a gas reference cell is detailed. This sets up the technique used to characterize longer-term wander. The test setup used to characterize wander is shared and methods are detailed. Results are reported peak-to-peak wander over minutes, hours, and days following a laser calibration routine. Correlation with temperature is reported. A newer, upgraded generation VT-DBR system is tested and results reported.

\subsection{VT-DBR Sweep Calibration}

The VT-DBR system monitors the optical frequency sweep and employs internal algorithms to optimize certain parameters of a sweep [6]. The details of these proprietary algorithms and calibration methods may not be detailed, as requested by Insight Photonic Solutions.

Through the Insight Laser control software, a user may initiate a calibration of the optical frequency sweep at any time. This is called a "sweep calibration." The user may perform either a baseline sweep calibration or a self-calibration.

A baseline calibration routine creates a sweep across the entire wavelength or optical frequency range of the laser $(1522.73-1563.71 \mathrm{~nm}$ for the module in this project) with the maximum number of points possible. This results in a linear optical frequency sweep with respect to time. The linear equation describing the laser sweep has a slope of approximately $105 \mathrm{MHz}$ per $2.5 \mathrm{~ns}$, referred to as the optical frequency step, and an offset equal to the minimum wavelength of the sweep.

A self-calibration is performed when custom sweep parameters are desired. 
Parameters that may be modified include but are not limited to output power, power profile, minimum sweep wavelength, maximum sweep wavelength, sweep repetition rate, duty cycle, number of points per sweep, optical frequency step, and start sweep trigger edge.

Following sweep calibration (baseline or self), the laser software reports all of the laser settings. These values may deviate slightly from the values entered by the user as the calibration routine optimizes sweep parameters.

The internal calibration algorithm does not contain any absolute wavelength reference. Therefore, we use an external gas reference cell (DUT 1, figure 4-5) to verify the accuracy and reliability of the laser's internal routine. Minimum wavelength and optical frequency step of the sweep are the parameters that are used to define the accuracy of any calibration.

The goal of this analysis is to quantify how much these laser parameters deviate over longer periods of time - especially the optical frequency step and minimum offset wavelength.

\subsection{Calibration Test Setup}

The test setup used to record sweeps, calibrate and measure VT-DBR optical frequency wander is very similar to the setup used for jitter characterization from chapter 4 (figure 4-1). The VT-DBR system is user-controlled through the Atlas laser control software. The optical frequency sweep is immediately attenuated to prevent photo detector saturation. In this setup, the only device under test is a molecular absorpt ion based wavelength reference or gas absorption cell (DUT 1 from jitter characterization). Other DUTs are not used. The optical signal is then coupled onto an amplified photo 
detector. The modulated RF output of this detector is low pass filtered and recorded by the high-speed digitizer. The oscilloscope is used for temporal signal alignment.

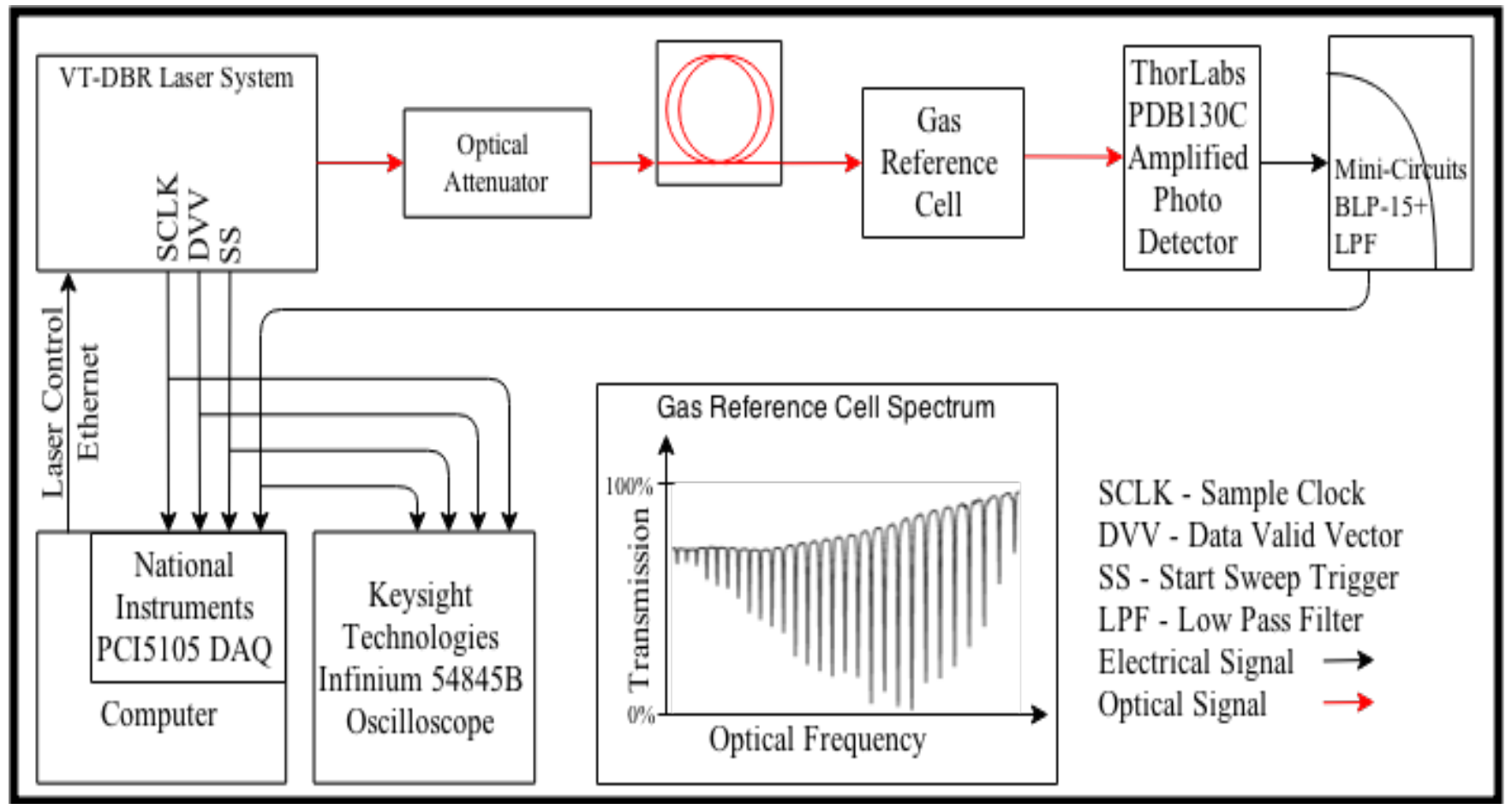

Figure 5-1 - Block diagram of test setup used to monitor wander of the VT-DBR optical frequency sweep. This setup is similar to the jitter measurement setup; the only devices under test in this experiment are gas absorption reference cells. 
For this aspect of the project, significant work was dedicated to the automation of data acquisition. Unlike jitter measurements which should be taken over a very short range of time (seconds or less), wander should be recorded over minutes, hours, or even days.

One option that was considered is continuous data acquisition over the time of interest (1 day, for example). This may be suitable for minutes or even several hours of data, but this method generates an extremely large amount of data since acquisition takes place continuously. Ultimately, the data sets generated by continuous recording occupy hundreds of gigabytes of data which limits the length of data acquisition. Not only does this make the maximum time of acquisition limited by the size of the computer's hard drive, it makes the post-processing of these data sets more tedious and lengthy. For this reason, continuous data acquisition could not be implemented.

To overcome this challenge, National Instruments (NI) LabView software was used to develop a modular, automated data acquisition system, allowing for flexible, pre-

\begin{tabular}{|ll|}
\multicolumn{1}{|c|}{ Parameter } & \multicolumn{1}{c|}{ Description } \\
\hline $\begin{array}{l}\text { Channel(s) from } \\
\text { which to Acquire } \\
\text { Data }\end{array}$ & $\begin{array}{l}\text { It may not be necessary to record data from all channels. For } \\
\text { example, a trigger or sample clock may not need to be recorded } \\
\text { and saved. This allows a user to save memory. }\end{array}$ \\
\hline $\begin{array}{l}\text { Length of Data Set } \\
\text { to be Acquired (in } \\
\text { \# of samples) }\end{array}$ & $\begin{array}{l}\text { The user may tune the acquisition such that only the data of } \\
\text { interest is recorded per sweep. }\end{array}$ \\
\hline $\begin{array}{l}\text { Number of Sweeps } \\
\text { to Save }\end{array}$ & $\begin{array}{l}\text { The user selects the number of sweeps to save over an entire data } \\
\text { acquisition. For example, if a sweep is captured each hour and a } \\
\text { day's worth of data is desired, 24 sweeps should be saved. }\end{array}$ \\
\hline $\begin{array}{l}\text { How Often to Save } \\
\text { a Sweep }\end{array}$ & $\begin{array}{l}\text { The sampling interval may be set, for example, to each minute, } \\
\text { hour, or any increment of time. }\end{array}$ \\
\hline
\end{tabular}

Table 5 - Parameters that are adjustable in the developed data acquisition system using NI LabView to make data collection automated and feasible. 
defined data collection. This system allows the user to program several parameters of a data acquisition, as shown in table 5.The NI PCI-5105 60 MS/s, 12-bit, 8-channel oscilloscope/digitizer was used and interfaces well with LabView.

The inputs to the NI PCI-5105 include the 3 signals generated by the laser module for synchronization (start sweep trigger, data valid vector, and the sample clock) and the RF output of the photo detector, which directly monitors the optical sweep of the laser.

The first step in characterizing wander is to determine a way to monitor single wavelengths of the laser sweep. Gas absorption cells play a critical role here for several reasons.

\subsection{Gas Cell Calibration}

To independently verify the quality of the internal calibration routine of the VTDBR, a highly-stable molecular absorption-based wavelength reference gas cell is employed. Gas cells are ideal for absolute wavelength accuracy and long-term stability [13].

A typical gas cell contains one or several highly pure gas compounds. Light is passed through this gas and depending on the gas as well as the wavelength of light, an absorption spectrum is generated. A typical spectrum exhibits sharp absorption lines at specific wavelengths. The absorption wavelengths correspond to fundamental vibrational and rotational energies of the gas molecules in the cell. This is why the absorption frequencies are so well-defined and stable over time [13].

Gas reference cells provide extremely accurate wavelength reference marker with up to $+/-0.1 \mathrm{pm}$ absolute accuracy [14]. Even when exposed to environmental factors such as temperature, pressure, humidity, or strain, these gas cells do not deviate 
significantly. For example, the gas cells drift at approximately $0.01 \mathrm{pm}$ per degree Celsius [14].

In order to independently calibrate a waveform, a single sweep must is acquired for analysis. The laser is coupled into a gas reference cell and the absorption spectrum is recorded. The data acquisition system must be triggered from the start sweep signal of the laser and aligned properly with the data valid signal to successfully carry out an external calibration. The data acquisition system samples as many sample points as possible. A raw laser sweep may then be processed and calibrated.

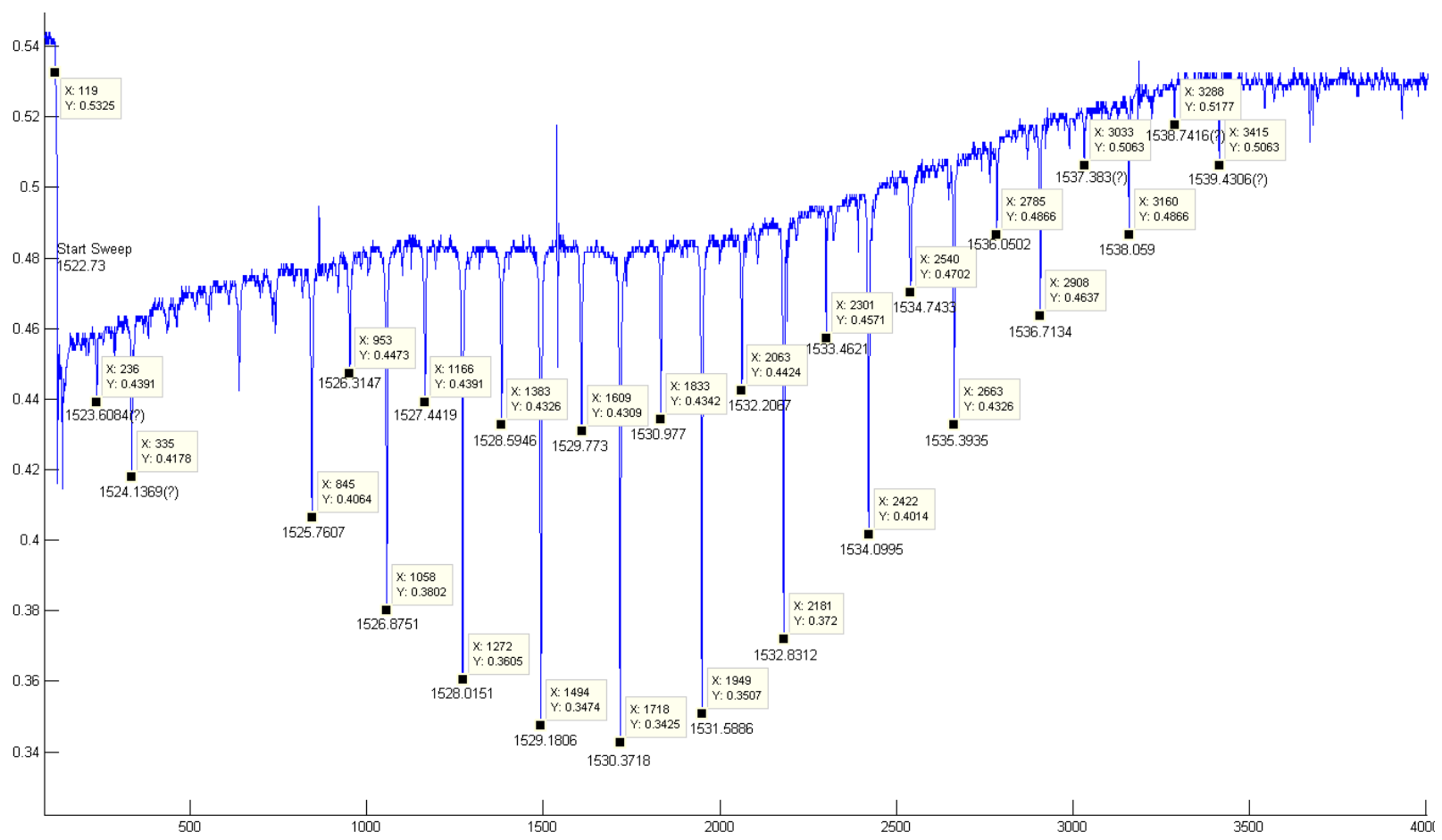

Figure 5-2 - An actual wavelength sweep in the range of the quad gas cell. The local minima which are labeled denote absorption peaks. These peaks have well-defined wavelengths associated with them. 


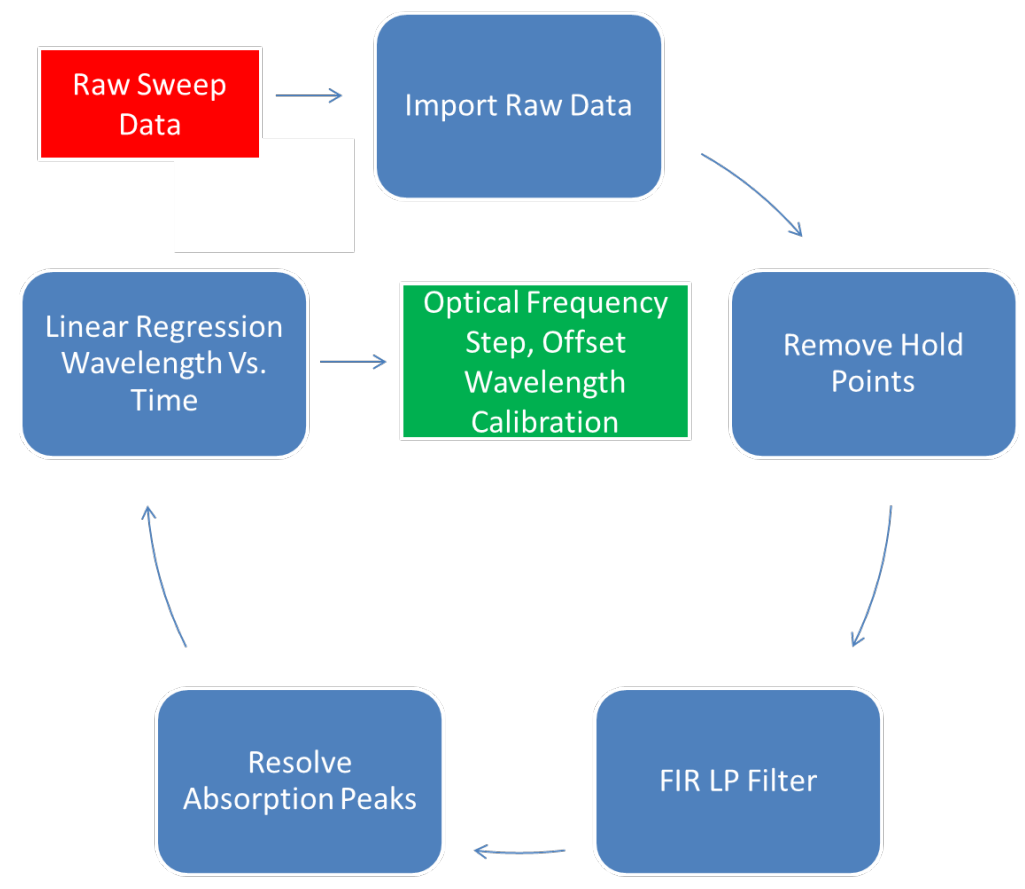

Figure 5-3 - The calibration algorithm takes raw sweep data as an input and first removes hold points based on the data valid vector. This signal is then low-pass filtered to remove high frequency noise. Finally, absorption peaks are identified and assigned a wavelength [14]. A linear regression is performed on the wavelength vs. time data to obtain the calibration parameters: optical frequency step and offset wavelength.

A Matlab algorithm was developed to process obtained sweep data. This

algorithm contains several important functions in order to obtain a highly accurate optical frequency step and minimum or offset wavelength for a given set of sweep data.

\subsection{Wander of the VT-DBR}

The calibration algorithms attempt to produce a sweep that is identical over long periods of time without drift or degradation. In investigating wander, we quantify how well the internal calibration mechanisms maintain their linearity (optical frequency step) and minimum or offset wavelength over time. 


\section{Model}

Wavelength Range

Table 6 - Gas cells used in this project. The WA-900-1638 is used to characterize wander due to its lower number of absorption features in the range of the VT-DBR sweep.

Gas reference cells are an ideal tool for long term monitoring of wavelength locations and drift. Most standard interferometers are extremely sensitive to ambient temperature, strain, humidity, or pressure because they are fiber-based. Thus, over time, we would not be able to differentiate laser system error to errors induced by environmental changes. Interferometer-based solutions were explored, but ultimately abandoned for these reasons. The gas cell, however, remains nearly independent of these external factors over long periods of time.

To monitor wander, the automated data acquisition system records sweeps of the laser at a preset interval. This process is undertaken over short intervals of 1 minute to 1 hour. The data is acquired periodically for hours and days.

Early tests involved monitoring individual absorption peaks and observing their shift in time relative to the start sweep trigger or other absorption peaks. 


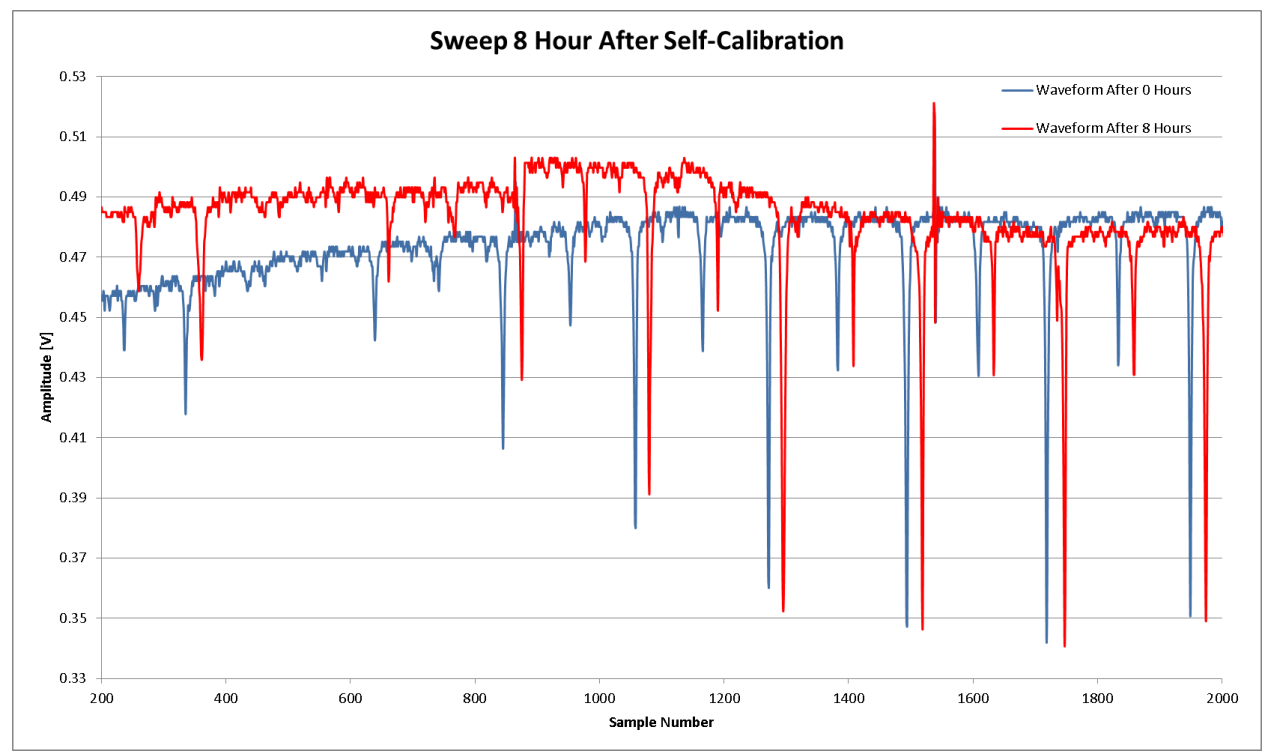

Figure 5-4 - Sweeps acquired at initial self-calibration, 10 minutes, 1, 2, 4, and 8 hours after. It is noted that the beginning of each sweep rises dramatically in amplitude over time. It is very easy to identify absorption peak drifts as well.

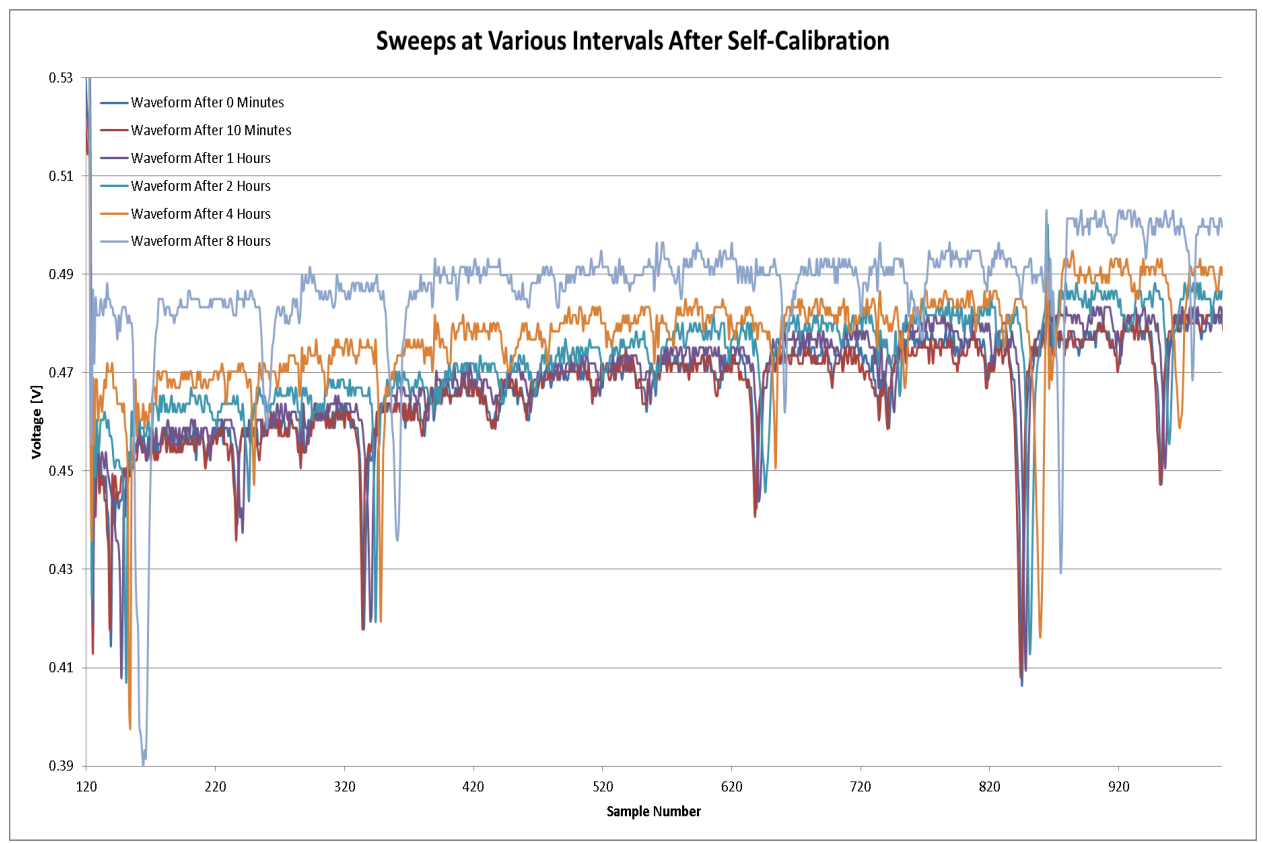

Figure 5-5 - The blue sweep was recorded directly following a self-calibration and the red sweep was recorded 8 hours later. There is a clear shift of the absorption peaks with respect to time. A deviation in amplitude is also noted. 
These initial data suggest that intrinsic wander to the laser source exists and should be further investigated. Now, instead of monitoring single peaks, each visible, well-defined absorption peak is analyzed and a linear regression is performed on the time, wavelength pairs.

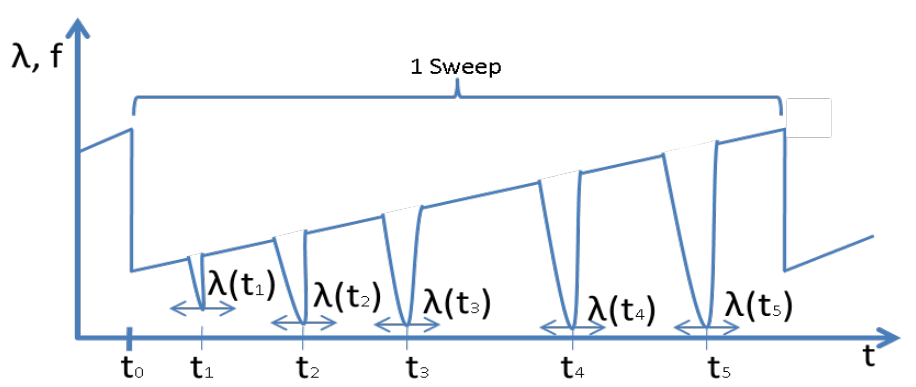

Figure 5-6 - Once each absorption peak has been identified in time, these wavelength, time coordinates are plotted to form a linear regression model of the optical frequency sweep. This figure shows only 5 peaks, but actual linear regression is based off of 28 absorption peaks.

Once a linear regression has been performed on a sweep, the sweep may be characterized by its slope or optical frequency step and its offset or minimum wavelength.

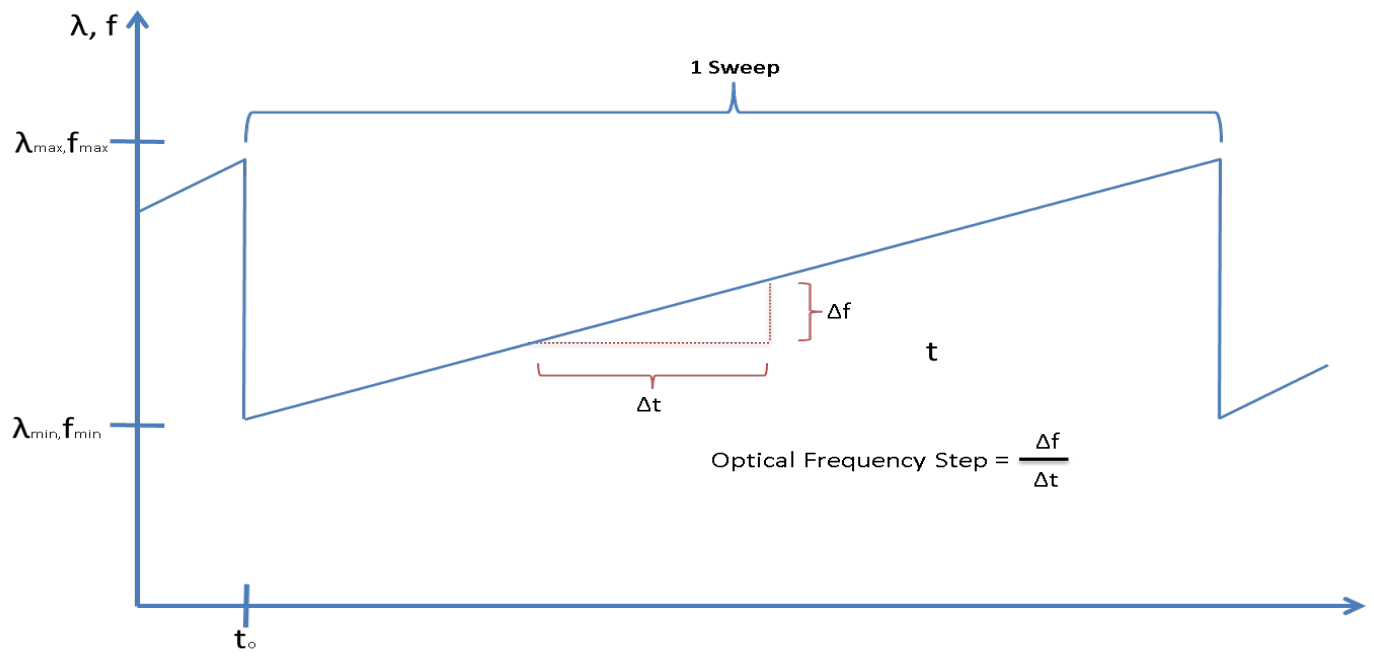

Figure 5-7 - The locations of absorption peaks are plotted with their corresponding optical frequency to obtain a linear regression model of the sweep. This model supplies the optical frequency step and minimum wavelength of the sweep. 
Data are presented which monitor the optical frequency step of the laser sweep as well as the minimum offset wavelength of the sweep over several periods of time.

These data show some particularly interesting behavior. First, with regard to the optical frequency step, we observe a peak-to-peak difference of no more than $62 \mathrm{kHz}$. We state that an observed optical frequency step is $105.25 \mathrm{MHz}+/-31 \mathrm{kHz}$. It is noted that the standard deviation or RMS value of the uncertainty is no more than $13 \mathrm{kHz}$. This is very low and approaches the limits of gas cell accuracy of $0.3 \mathrm{pm}$.

Moreover, no consistency is observed in the data seems to suggest that the fluctuations are due to external factors. Fluctuations are likely a function of random noise in the laser cavity and drive currents. This noise may originate from analog to digital or digital to analog conversions in the drive circuitry.

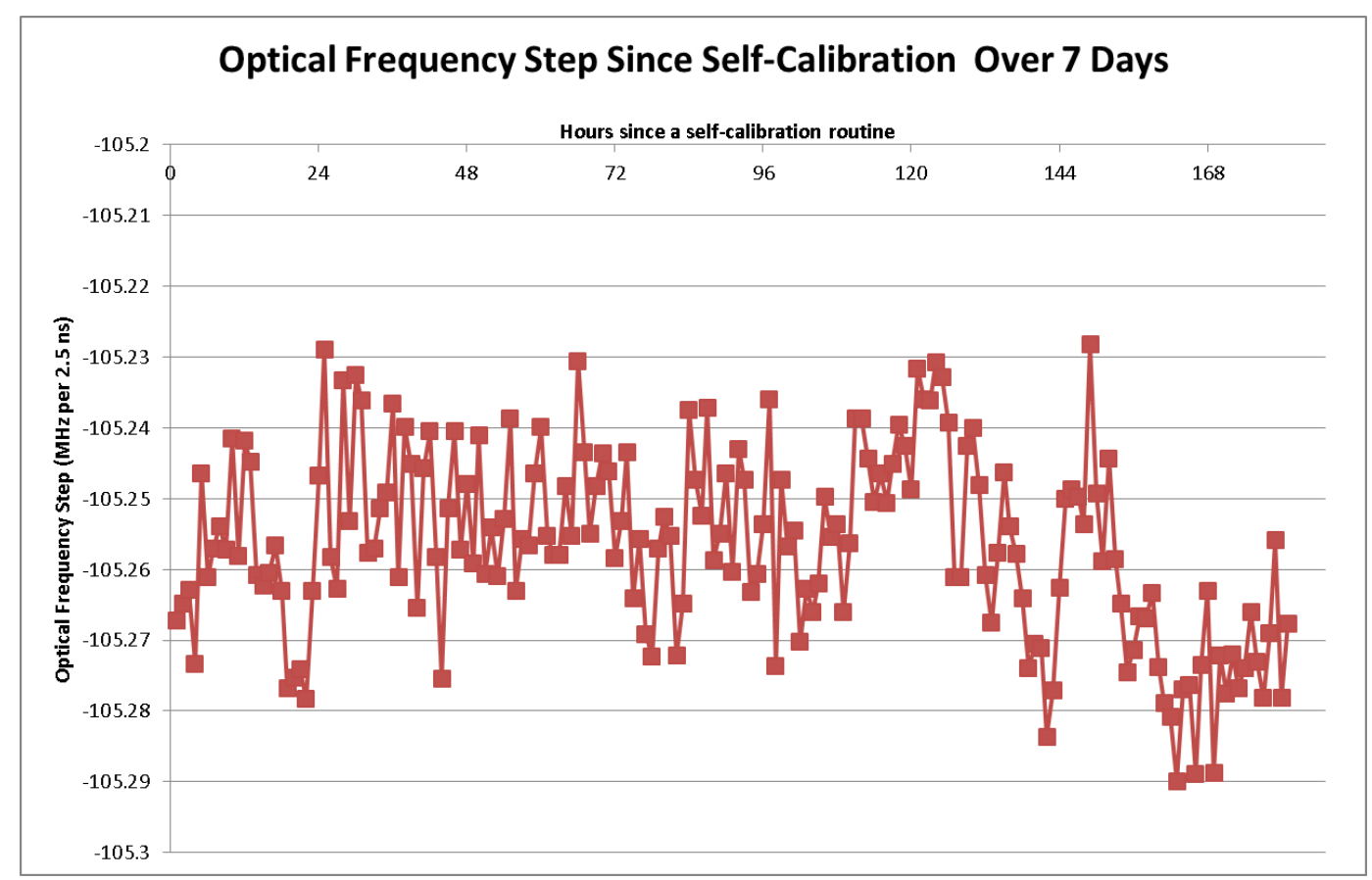

Figure 5-8 - Optical Frequency Step over 7 days. The fluctuations are extremely minimal and do not have any sort of noticeable pattern to them. 
With regard to the minimum wavelength of the sweep, a somewhat periodic shape is observed with an approximate period of 1 day. A maximum deviation (peak-to-peak) of $358 \mathrm{pm}$ is observed. The RMS value is $70 \mathrm{pm}$. This data is shown in figure 5-9.

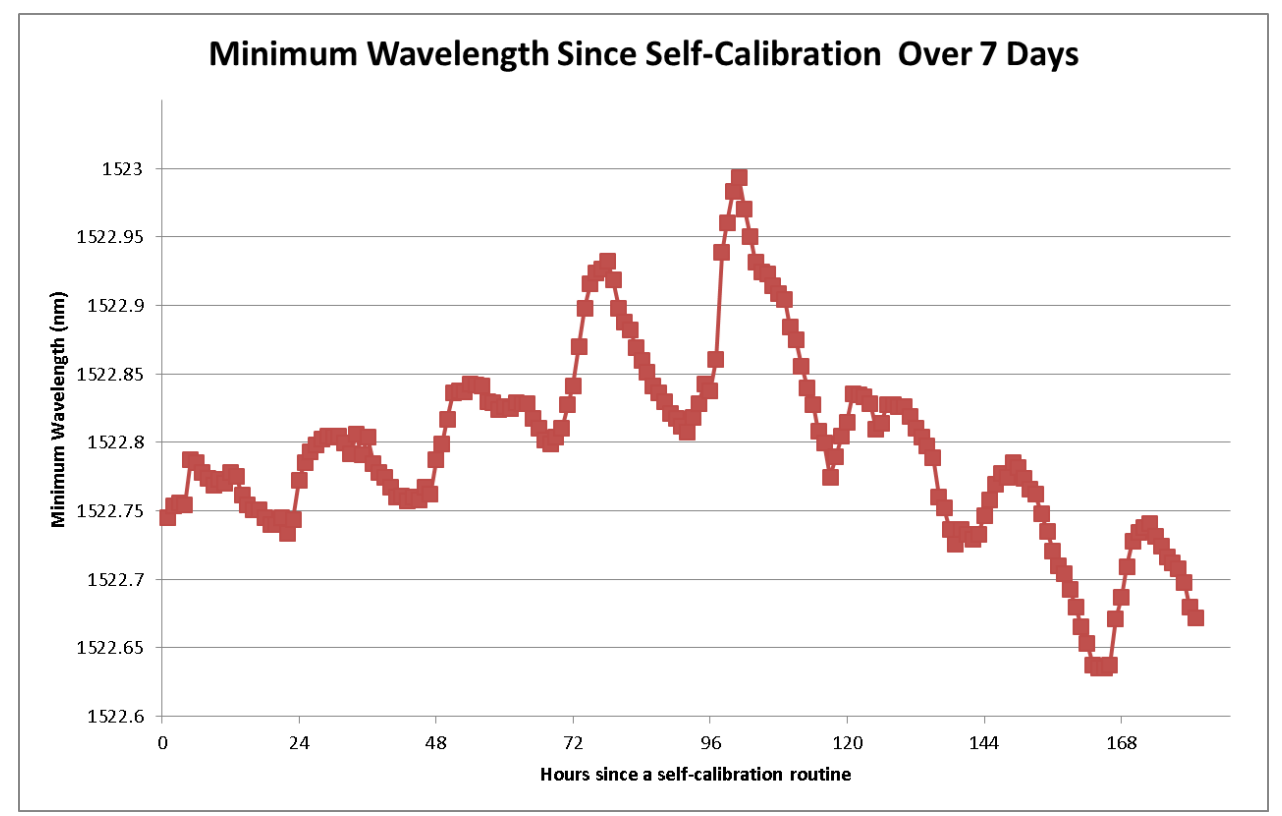

Figure 5-9 - The minimum sweep wavelength recorded each hour for 7 days. Data suggests that there is some mechanism controlling the somewhat periodic nature of the wavelength.

The periodic nature of the minimum wavelength with respect suggests an external factor may be influencing this source. This is because we know that the gas cell is highly stable as is our photo detector. Thus, we can attribute drift to the laser source itself and not the measurement device.

Temperature is proposed as a factor that might lead to such variations. To test this theory, a thermocouple is added to the test setup to measure the temperature of the case over the data collection. This thermocouple is applied to the top of the laser case using thermal grease to maintain a consistent measurement. 


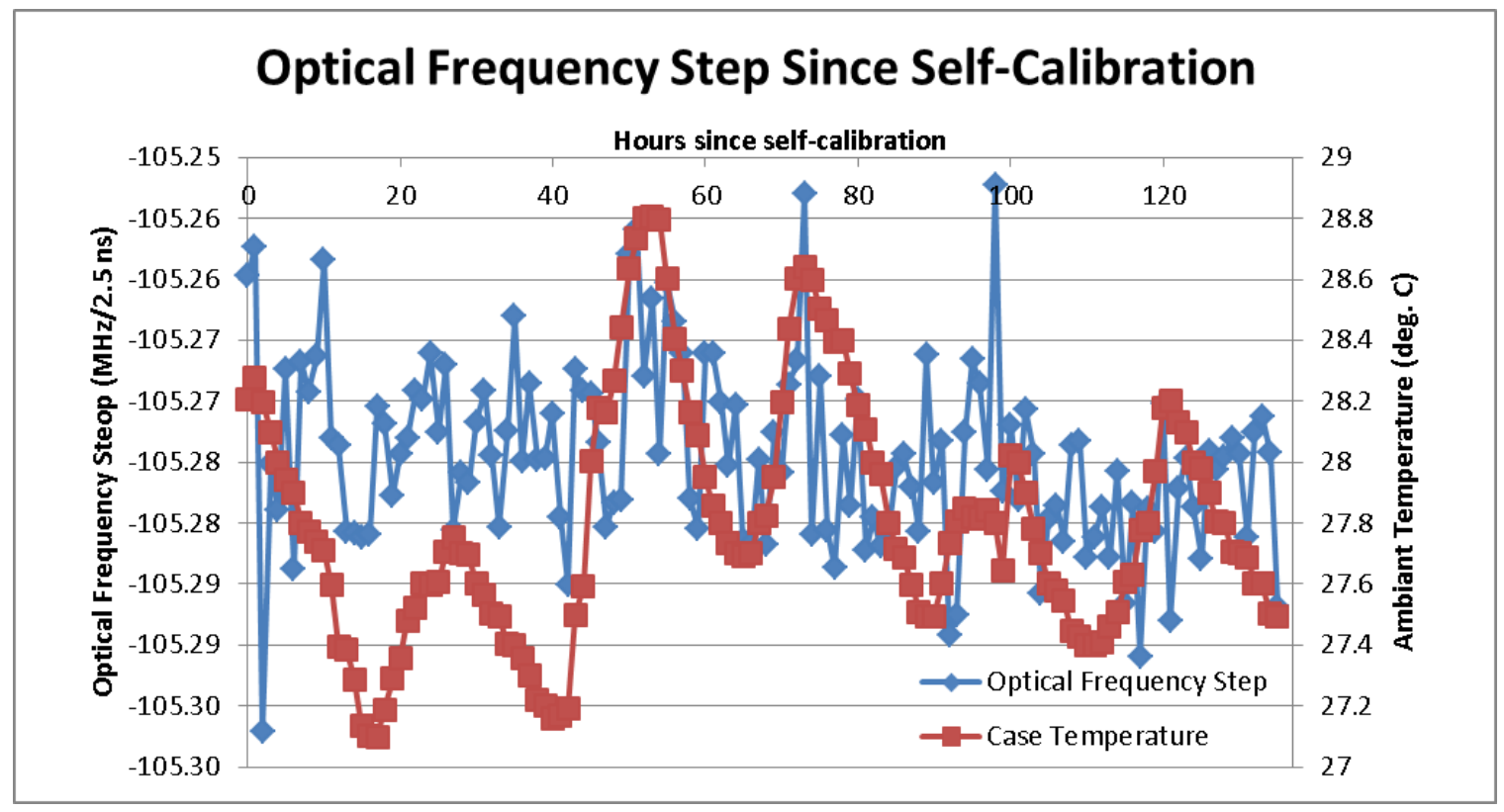

Figure 5-10 - Optical frequency step monitored over 138 hours since a self-calibration with monitoring of case temperature. No significant correlation is observed and the optical frequency step remains very consistent.

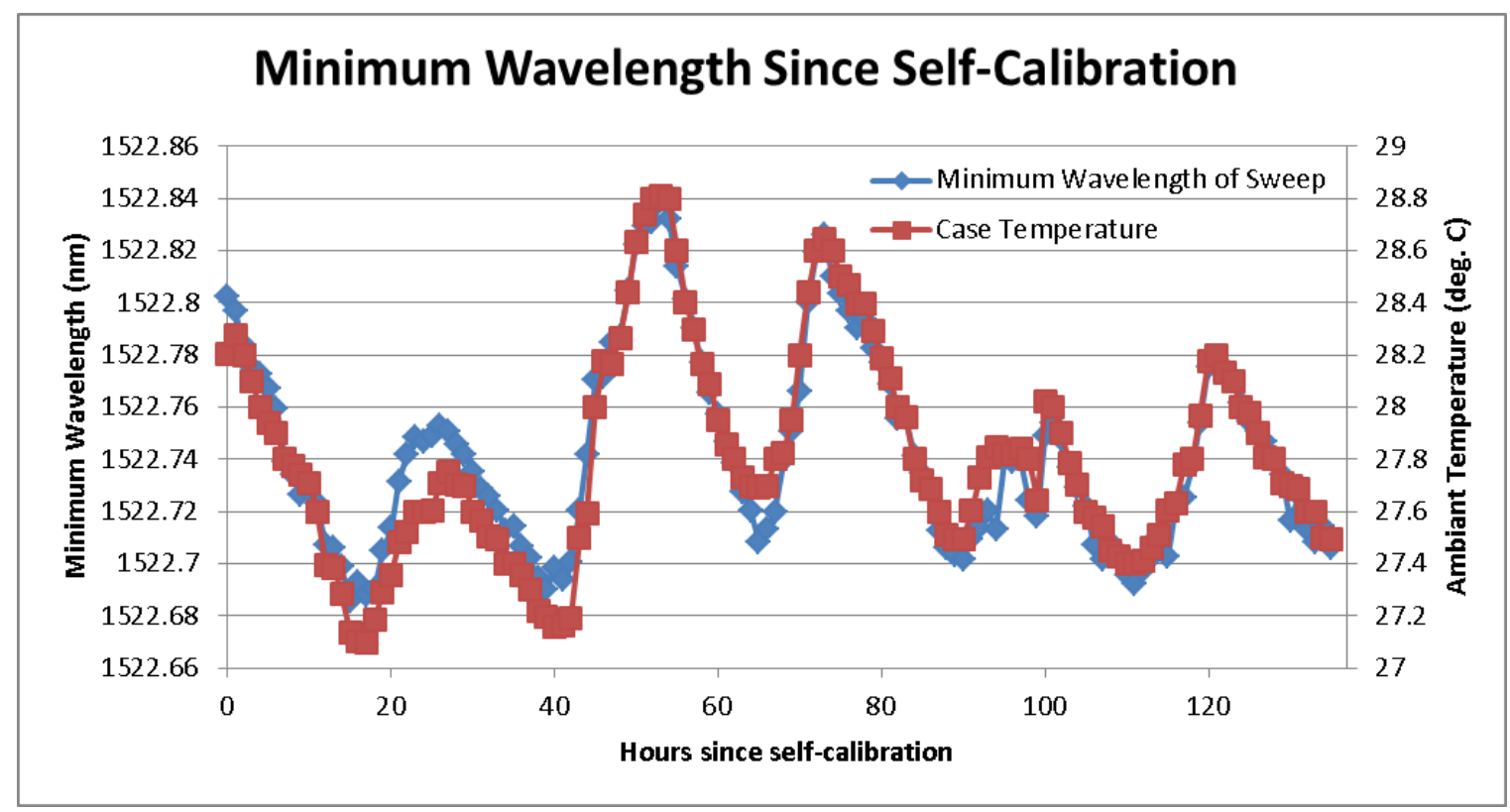

Figure 5-11 - Minimum Wavelength of linear fit monitored over 138 hours following a self-calibration. Strong correlation with case temperature is observed. 
This data set offers more insight into the behavior of the VT-DBR system while also reinforcing previous results obtained without temperature monitoring. First, the optical frequency step remains extremely consistent, deviating by no more than $45 \mathrm{kHz}$ peak-to-peak or $7.5 \mathrm{kHz}$ RMS over 120 hours following a self-calibration. This deviation is negligible and shows the high quality linearity maintained by the VT-DBR source over long periods of time and with external temperature deviations. Case temperature does not appear to cause fluctuations in optical frequency step.

As proposed, the data shows that temperature may be a significant factor contributing to the periodic nature of the minimum offset wavelength of the laser sweep. Over nearly 5 days, the measured case temperature directly correlates to the offset wavelength. This data set exhibits a peak-to-peak wavelength drift of $154 \mathrm{pm}$ with a corresponding 37 pm RMS drift. This occurs over a temperature range of 1.708 degrees Celsius.

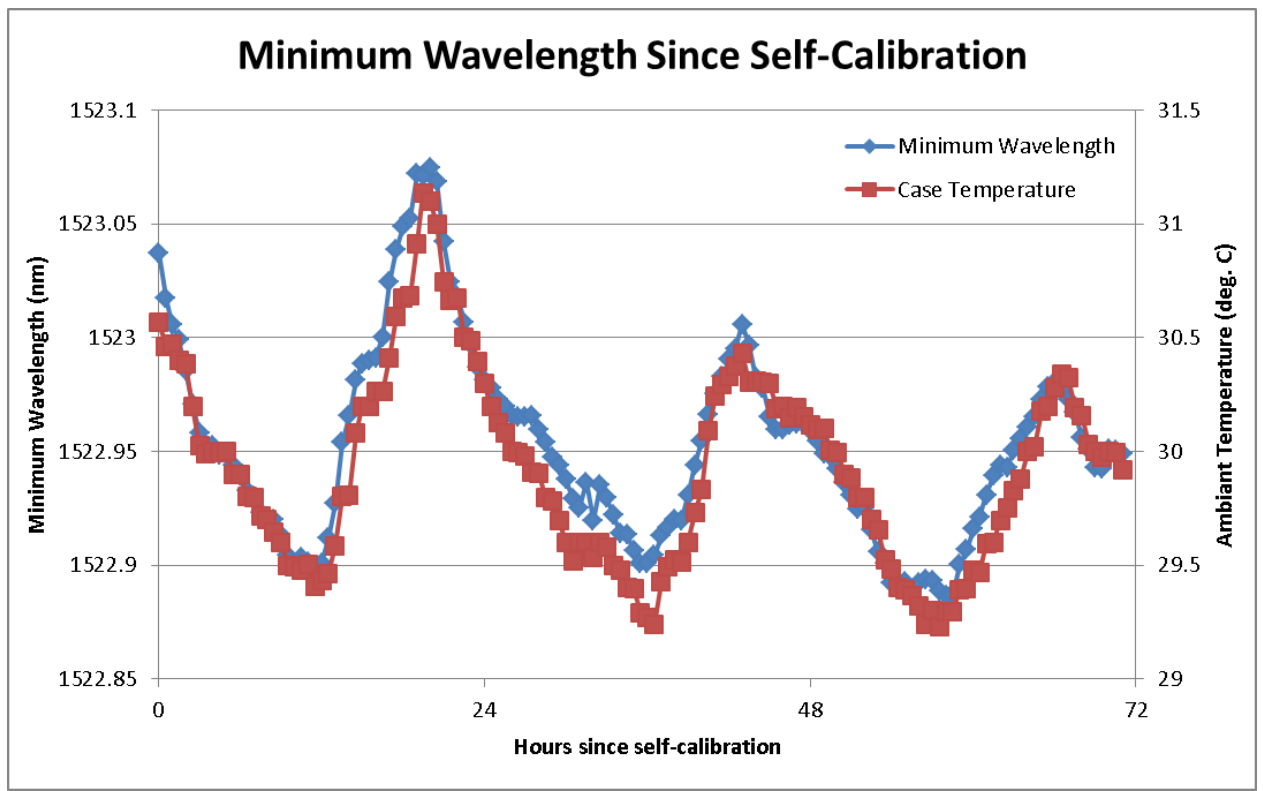

Figure 5-12 - Another data set displays similar behavior. The minimum sweep wavelength is directly correlated with ambient case temperature. 
Figure 5-13 once again confirms a correlation between temperature and minimum wavelength of the sweep. This data set reports a maximum peak-to-peak deviation of 188 pm and 43 pm RMS over 1.908 degrees Celsius temperature range.

A similar phenomenon is observed over a shorter time period, at shorter 1 minute sampling intervals (instead of 1 hour intervals). Figure 5-14 shows the offset wavelength and temperature for the first 2 hours after a self-calibration. Again, it is clear that the offset wavelength is a function of the ambient temperature. Here we observe a $369 \mathrm{pm}$ drift in offset wavelength over a 5.467 degree Celsius climb in temperature.

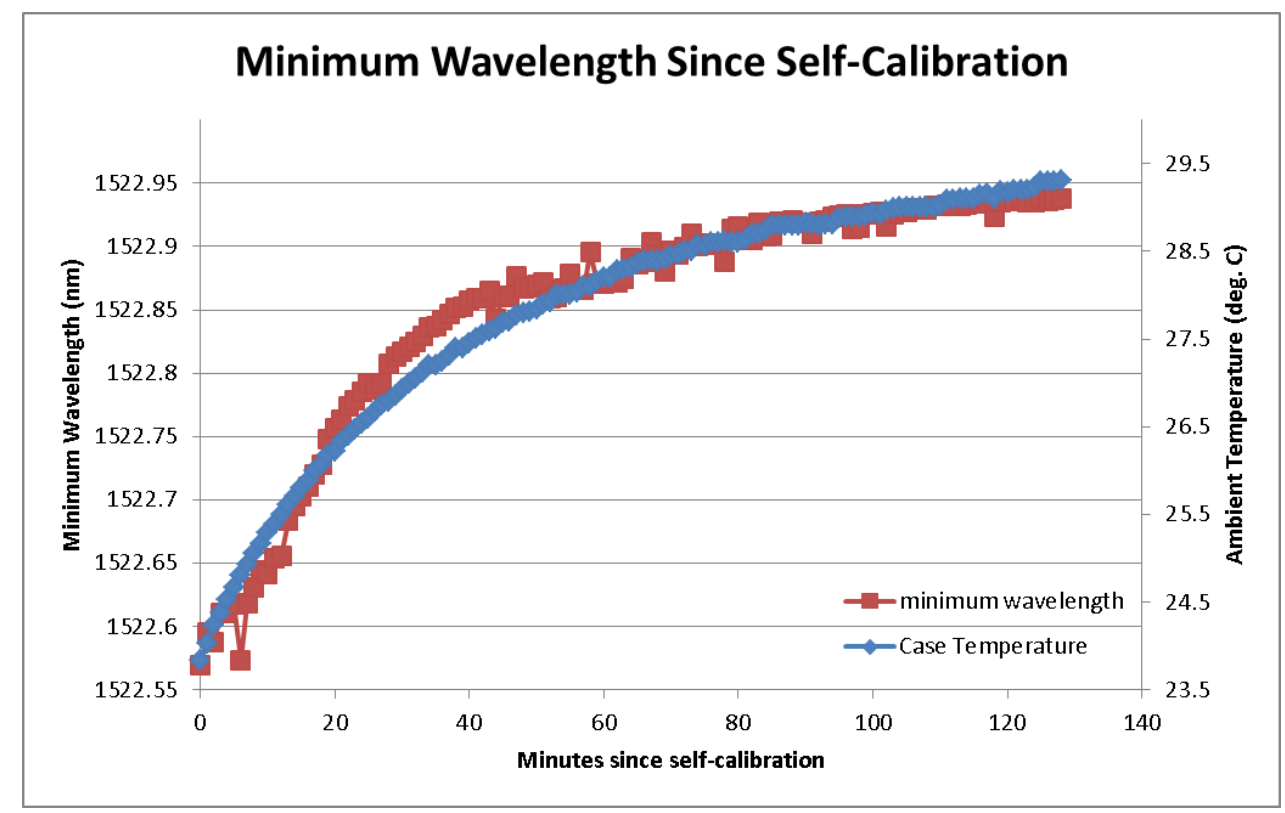

Figure 5-13 - Minimum wavelength recorded each minute for just over 2 hours with temperature data alongside. 


\subsection{Wander of a 2nd Generation VT-DBR}

The same $2^{\text {nd }}$ generation VT-DBR module tested in section 4.3 for jitter is now tested for longer-term wander. Preliminary results with the $2^{\text {nd }}$ generation unit which contains upgraded drive circuitry and noise reduction mechanisms shows significant improvements upon the longer-term variations of the laser sweep. Significantly improved drift is reported in this module.

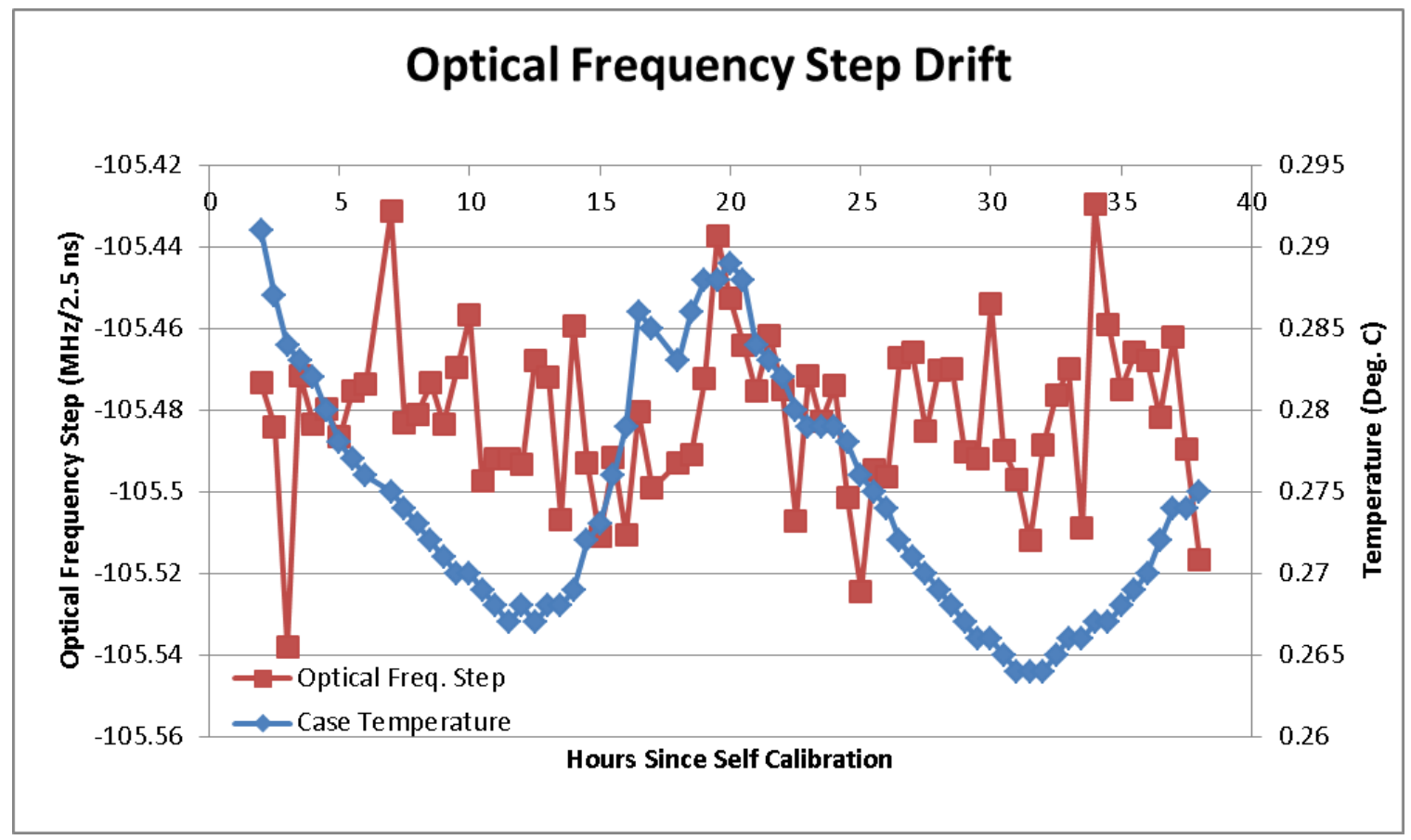

Figure 5-14 - Optical frequency step of 2nd generation VT-DBR laser system over 38 hours of data collection. A highly stable optical frequency step is observed over the data collection period as in the previous module.

As with the previous module, the new revision maintains a highly stable optical frequency step over hours and days of time as well as case temperature fluctuations of several degrees Celsius. An optical frequency step on the order of $105 \mathrm{MHz}+/-10 \mathrm{kHz}$ RMS per $2.5 \mathrm{~ns}$ is recorded over a temperature range of $2.7^{\circ} \mathrm{C}$ and a data collection 
period of 38 hours. This confirms that the linear optical frequency sweep is still highly stable in the newer revisions of the VT-DBR system.

With regard to the drift in absolute wavelength, the new module shows significant performance improvement. Over the same 38 hour data collection period, the laser minimum or offset wavelength drifts by no more than 16.3 pm peak-to-peak. Moreover, these variations do not appear to be correlated with case temperature as in the previous module.

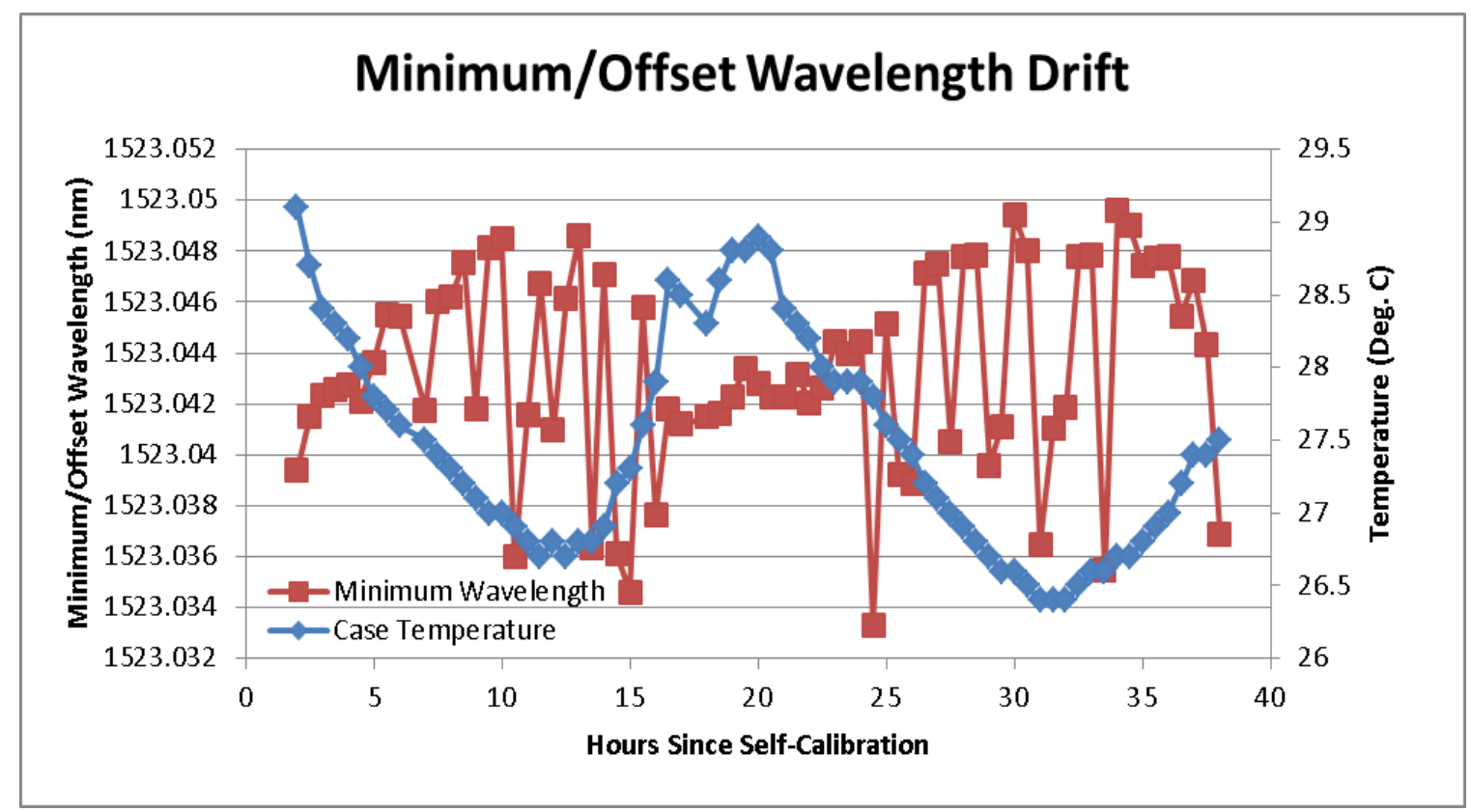

Figure 5-15 - Wavelength wander of 2nd generation VT-DBR laser system over 38 hours of data collection. The new module exhibits significant performance improvements over the first VT-DBR system tested. No correlation with case temperature is observed. 


\subsection{Analysis}

Having quantified the drift in optical frequency or wavelength offset of the linear sweep, many questions exist about the VT-DBR. First, we summarize findings. Postanalysis is completed on data sets, causes are explored, and implications on fiber sensing applications are addressed. Through the course of wander data collection, the results in table 6 were obtained over several trials in the first VT-DBR module under test. These data and the experiments from which they were derived confirm several findings.

\begin{tabular}{|l|l|l|l|l|l|l|}
\hline $\begin{array}{l}\text { Collection } \\
\text { Period }\end{array}$ & $\begin{array}{l}\text { Sampling } \\
\text { Interval }\end{array}$ & $\begin{array}{l}\text { Peak-to-Peak } \\
\text { ptical } \\
\text { Frequency }\end{array}$ & $\begin{array}{l}\text { RMS } \\
\text { Optcal } \\
\text { Frequency }\end{array}$ & $\begin{array}{l}\text { Peak-to-Peak } \\
\text { Minimum } \\
\text { Wavelength }\end{array}$ & $\begin{array}{l}\text { RMS } \\
\text { Minimum } \\
\text { Wavelength }\end{array}$ & $\begin{array}{l}\text { Peak-to-Peak } \\
\text { Temperature } \\
\text { Deviation }\end{array}$ \\
\hline 96 Hours & 6 Hours & $93.2 \mathrm{kHz}$ & $24.4 \mathrm{kHz}$ & $224.4 \mathrm{pm}$ & $71.5 \mathrm{pm}$ & not available \\
\hline 91 Hours & 1 Hour & $54.4 \mathrm{kHz}$ & $14.5 \mathrm{kHz}$ & $317.6 \mathrm{pm}$ & $65.1 \mathrm{pm}$ & $2.492 \mathrm{C}$ \\
\hline 72 Hours & 30 Minut & $51.6 \mathrm{kHz}$ & $9.7 \mathrm{kHz}$ & $188.1 \mathrm{pm}$ & $42.6 \mathrm{pm}$ & $1.908 \mathrm{C}$ \\
\hline 2 Hours & 1 Minute & $0.7 \mathrm{kHz}$ & $0.2 \mathrm{kHz}$ & $368.8 \mathrm{pm}$ & $98.1 \mathrm{pm}$ & $5.467 \mathrm{C}$ \\
\hline 181 Hours & 1 Hour & $61.6 \mathrm{kHz}$ & $13.3 \mathrm{kHz}$ & $358.3 \mathrm{pm}$ & $69.4 \mathrm{pm}$ & not available \\
\hline 135 Hours & 1 Hour & $44.8 \mathrm{kHz}$ & $7.6 \mathrm{kHz}$ & $153.6 \mathrm{pm}$ & $37.2 \mathrm{pm}$ & $1.708 \mathrm{C}$ \\
\hline
\end{tabular}

Table 7 - Summary of Wander results in the 1st generation VT-DBR laser module under test over several data collection periods. Consistent results correlate temperature with offset wavelength and a demonstrate a very stable optical frequency step.

First, it is noteworthy that the optical frequency step is very robust over time. In fact, the numbers obtained by gas cell calibration are nearing the accuracy of the gas cell absorption line locations. These peak-to-peak and RMS optical frequency deviations are several orders of magnitude lower than the optical frequency step itself

Moreover, the optical frequency step does not appear to be influenced in any significant fashion by temperature of the laser at present. This means that the internal calibration routine of the source does in fact create a highly linear, repeatable optical frequency sweep that maintains linearity over temperature fluctuations and time. 


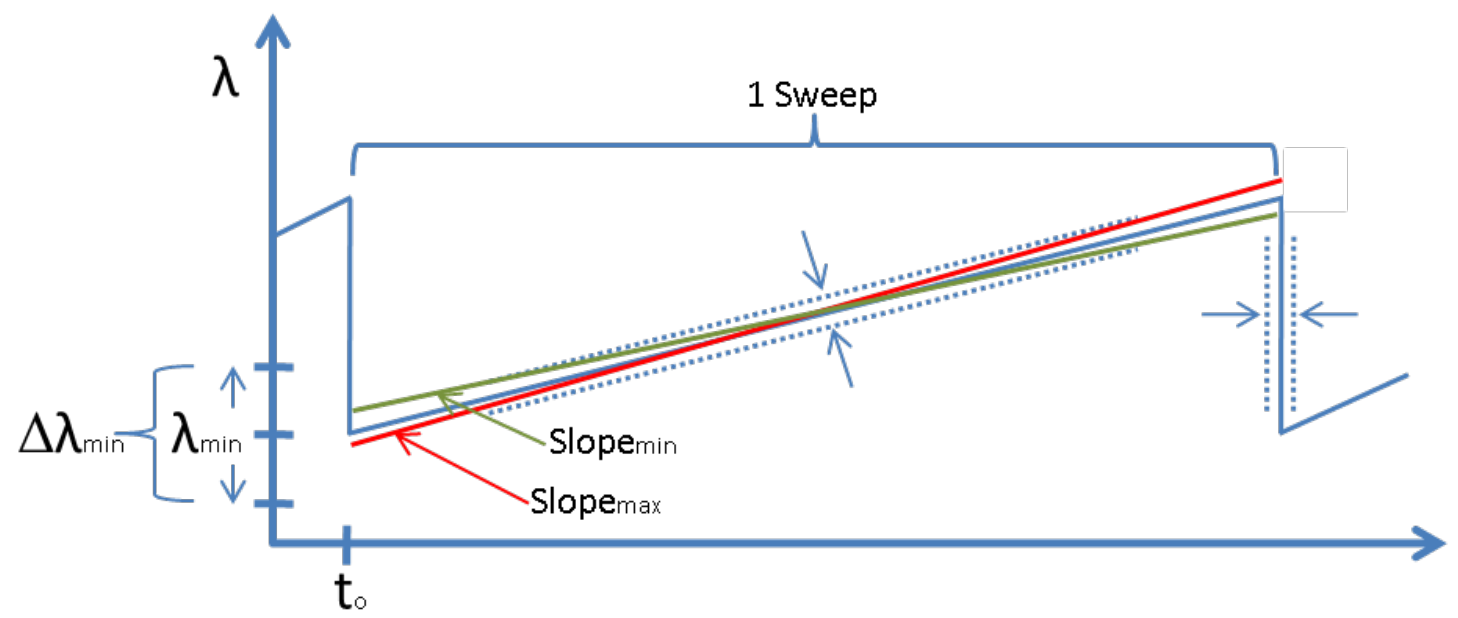

Figure 5-16 - The linear regression of a wavelength sweep ultimately provides the slope or optical frequency step of the sweep and the minimum wavelength or offset wavelength. A large range of wavelength offset is observed with regard to temperature while the slope stays very consistent, deviating only a small amount.

The data also provides much insight regarding the optical frequency offset of the sweep - the so-called minimum wavelength of the sweep. As demonstrated, the linear offset deviates significantly with changes in case temperature.

A scatter plot of wavelength versus temperature yields a set of data that appears to follow a linear distribution. By performing a linear regression on this data, a function is obtained to relate offset wavelength to case temperature. This yields an approximate temperature coefficient, in units of nm per degree Celsius.In two cases with over 100 sample points each, a temperature coefficient of around $90-100$ pm per degree Celsius is observed. 


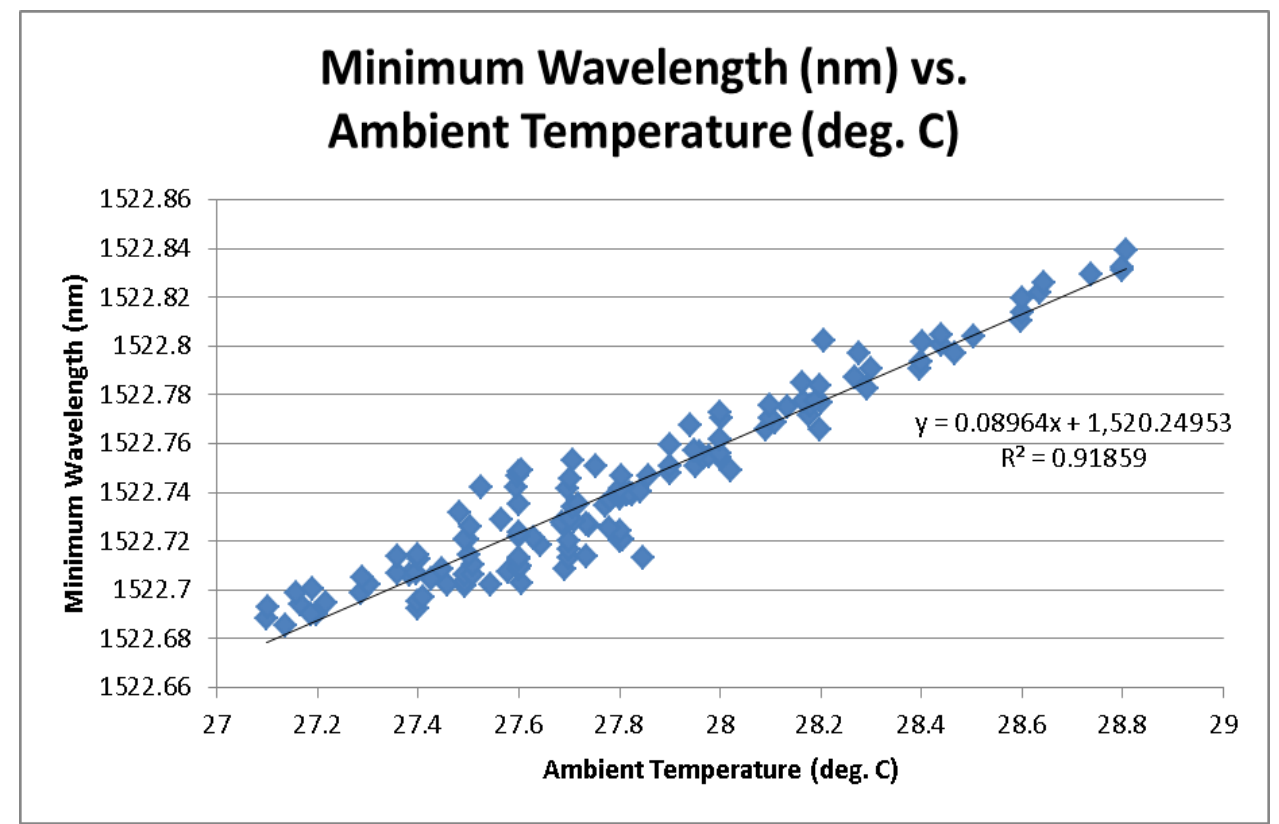

Figure 5-18 - Linear Regression performed on a data set of wavelength vs. ambient or case temperature. This yields a temperature coefficient of approximately $90 \mathrm{pm}$ per degree Celsius.

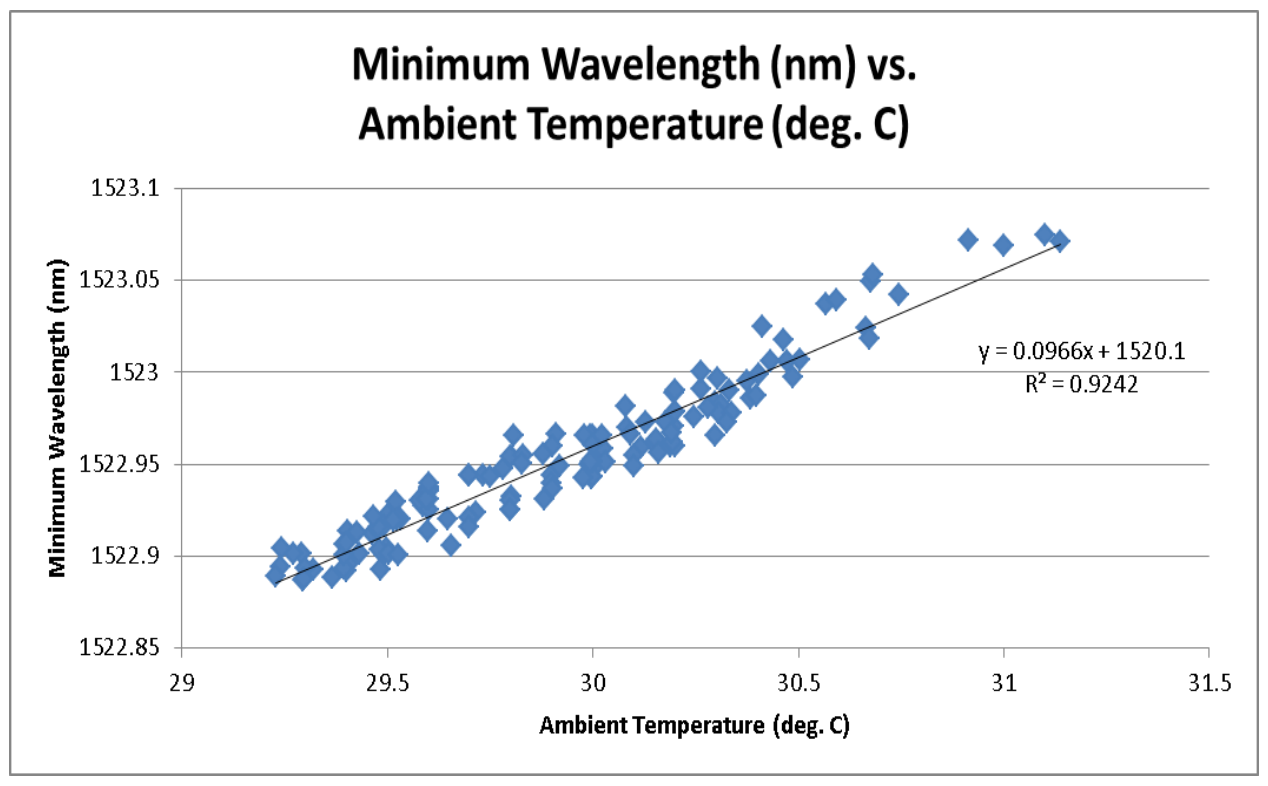

Figure 5-17 - Linear Regression performed on a data set of wavelength vs. ambient or case temperature. This yields a temperature coefficient of approximately 97 pm per degree Celsius. 
A temperature coefficient of approximately $120 \mathrm{pm}$ per degree Celsius was measured with only the packaged laser module and an ILX temperature controller. This means that the case temperature of the laser system case may be a representation of the actual laser temperature within its butterfly package.

In this case, the thermal electric cooler (TEC) which has the purpose of thermally regulating the laser chip could be malfunctioning or "unlocked." Furthermore, it could mean that the TEC contains some intrinsic temperature dependence itself which must be accounted for. These issues will be investigated.

Another potential cause of wavelength wander arises from the current drives of the laser. The possibility exists that the current drivers to the VT-DBRs 3 main modulation drivers, the front mirror, back mirror, and phase section are all drifting as a function of temperature and inducing a wavelength drift that is also temperature dependent. If the currents drift in magnitude ever so slightly, the wavelength is forced to shift as well.

In order to inspect this hypothesis, individual wavelength absorption peak locations were tracked over time to view the shifts in individual wavelengths. Each peak was analyzed to determine if it had a large temperature coefficient, denoting it as a sensitive region of the sweep.

While certain peaks do drift slightly more than others, there appears to be no consistent pattern. This sub-analysis of drift data does not immediately reveal that the drive currents are drifting as proposed.

Though wander on the order of hundreds of picometers has been observed, OCT methods may not be significantly impacted. This is for two main reasons. 
First, in OCT applications, it is the short term, high frequency stability (jitter) which affects performance. Jitter defines the maximum resolution of a scan and plays a significant role. Wander, however, does not have a significant impact on a scan. This is because the absolute wavelengths in an OCT scan are not as important. That is, information for a scan in nearly identical whether the scan begins at $1522.7 \mathrm{~nm}$ or 1523 $\mathrm{nm}$. It is the relative stability rather than the absolute stability which concerns a scan.

Second, OCT scans are generally executed within minutes or hours which suggests that the drift experienced in offset wavelength is negligible to OCT. If the VTDBR is acclimated to the room temperature and settings, the offset wavelength should drift by only a few picometers over the course of minutes to hours. This will not impact an OCT scan in any significant way.

In contrast, fiber sensing applications will be very sensitive to the wander that is reported in this chapter. Contrary to an OCT scan, sensor measurements and readings are generally referenced to an absolute wavelength.

For example, a fiber Bragg grating strain sensor will be calibrated to a certain strain at a well-defined wavelength. As strain increases or decreases, the wavelength will shift proportionally. Since sensors such as this one often sit idle for days if not months or years, we must be able to resolve the absolute wavelength of this sensor using our light source. If our source drifts over these long periods of time, our sensor measurements will be skewed and error will be induced by the source.

With hundreds of picometers of drift as observed in this project, the VT-DBR will not be able to resolve a wavelength shift to more than a few hundred picometers in its current state. Many FBG sensors respond to sub-picometer shifts wavelength. We 
conclude that the VT-DBR will require some sort of absolute wavelength reference or compensation before it is a viable source for fiber sensing applications.

Fortunately, the newer revision of the VT-DBR system eliminates the significant wander observed in the first module. We suggest that the observed wander in the first module was a result of a malfunctioning TEC because of the observed temperature coefficient.

The wander measurements from the new VT-DBR suggest that this source is viable for sensing applications such as FBG sensing. Moreover, the robustness and consistency of both the optical frequency sweep and absolute wavelength eliminate the need for an absolute wavelength reference which would be necessary if significant wander was present. 


\section{CONCLUSIONS}

A comprehensive study of the VT-DBR laser sweep stability has been undertaken. Findings, conclusions and implications with regard to the laser system as well as fiber sensing applications are presented.

\subsection{VT-DBR Laser}

In summary, intrinsic wavelength or optical frequency jitter and wander of the novel VT-DBR akinetic laser source has been characterized. A foundation for sweep calibration using an external gas reference cell is explored and provided.

The laser module under study in this project jitters in wavelength by no more than $1.57 \mathrm{pm}$ RMS or $196 \mathrm{MHz}$ in optical frequency. Average values are on the order of 0.5 pm RMS or about $60 \mathrm{MHz}$. Jitter appears to be slightly improved in the newer VT-DBR modules, potentially due to hardware improvements.

These measurements represent intrinsic linewidth of the source, which is no better than $10-20 \mathrm{MHz}$, as reported by Insight Photonic Solutions. It is expected that the measured linewidth will be larger than the nominal, 10-20 MHz value, since measurements were undertaken during dynamic tuning at high speeds rather than single mode, fixed-wavelength output.

Wander informs on the long-term stability of the source. A highly consistent linearity is reported around $105 \mathrm{MHz}$ per $2.5 \mathrm{~ns}$ which differs by no more than $+/-31 \mathrm{kHz}$ over the course of several days and ambient temperature fluctuations. These slight fluctuations in optical frequency step or linearity exhibit no consistency or correlation with temperature, time, or other factors. Similar results are found in the newest VT-DBR module with, once again, slight improvements to consistency. 
Contrary to the linearity, the offset or minimum wavelength of the sweep wanders significantly over time and ambient temperature in the 1st generation laser module under investigation. Several trials of data collection show a drift of up to $370 \mathrm{pm}$ in absolute wavelength of the laser sweep over several hours. This drift is strongly correlated with ambient case temperature. A temperature coefficient on the order of $100 \mathrm{pm} /{ }^{\circ} \mathrm{C}$ is recorded.

At present, the underlying mechanisms of wander are not understood. There are several possible reasons that the VT-DBR exhibits jitter and wander as observed. These reasons stem from the control, stabilization, and calibration mechanisms within the system. In the newest revision of the laser (2nd generation), wavelength wander has been nearly eliminated and no correlation with case temperature is observed. A maximum peak-to-peak fluctuation of about $16.3 \mathrm{pm}$ is reported in the new module.

Electronic drive circuitry is thought to contribute to intrinsic jitter observed in the present system. This may be further investigated by comparing wavelength jitter at different at higher versus low drive currents. Higher drive currents will likely exhibit a more significant degree of jitter. A certain amount of jitter or uncertainty in wavelength will always be present in such a laser device due to random noise. It may, however, be possible to reduce jitter to a smaller value if the mechanisms contributing to jitter are understood.

Since the VT-DBR is an SG-DBR type of laser, complex drive currents are required to create the high-speed, linear sweep. These currents should be jitter-free, but noise is may cause small fluctuations in the drive currents. These fluctuations will ultimately cause small fluctuations (jitter) in the output wavelength of the laser chip. 
Noise contributing to jitter in the system may develop in any part of the drive circuitry. Important sub-components to investigate include any transimpedance amplifiers, analog-to-digital converters, and digital-to-analog converters. The power supply for the VT-DBR may also contribute to this problem if it does not isolate the system from external electrical noise and create a superbly clean power source for the system.

Further investigation will be required to understand which mechanisms contribute most to jitter in the output wavelength. Due to the Gaussian nature of wavelength jitter, however, we conclude that stochastic processes do contribute to jitter to some degree. Some amount of wavelength jitter will always be present in the system. Underlying mechanisms causing wander differ from those responsible for jitter.

Drift of laser butterfly package itself is on the order of $120 \mathrm{pm} /{ }^{\circ} \mathrm{C}$ as reported by Insight Photonic Solutions. This suggests that measurement of case temperature as recorded in this experiment may reflect the actual temperature of the butterfly package of the actual laser within the module. A thermal electric cooler (TEC) is implemented to compensate for these small fluctuations in temperature of the laser chip, which suggests 2 causes: (1) the TEC is not operating correctly and becoming unlocked or (2) the TEC exhibits some intrinsic temperature dependence itself. We conclude that the TEC malfunctioned in the first unit under test since the temperature coefficient observed is very similar to the temperature coefficient of the laser butterfly package. The 2 nd unit exhibits almost no wander or correlation with case temperature.

Along with characterization of stability, this project proposes a method for externally calibrating a laser sweep in post-processing was developed. This method 
requires a laser sweep to be recorded through a gas reference cell which is not presently integrated into the VT-DBR system.

This project developed a preliminary algorithm for external calibration, implemented in a Matlab script. A calibration using this method provides 2 sweep metrics: the optical frequency step and the offset or minimum sweep wavelength. The calibration is qualified by the residuals observed between the linear fit and the actual recorded location of at least 28 wavelength markers from the gas cell.

Such a calibration provides an absolute wavelength reference for the VT-DBR. This is especially helpful to quantify and possibly compensate for any wander effects observed in this project through post-processing.

\subsection{Fiber Optic Sensing}

Fiber optic sensing encompasses applications where the sensing element is an optical fiber or fiber optics is used to relay signals and information from a sensor. Fiber optic sensing holds many benefits over traditional electrical sensors and shows promise for the future when applications become more demanding. Benefits include light weight and discrete size and packaging, independence from electrical signals and power, and the ability to multiplex many sensors in a single fiber.

In precision fiber optic systems, it is crucial to have a consistent and reliable light source. This ensures that readings from the system/sensor are due only to sensor modulation and not source jitter or drift.

In OCT applications, jitter has a stronger impact than wander. This is largely due to the fact that OCT scans are performed over short periods of time, so the effects of long term wander do not come into effect. Conversely, wavelength jitter limits resolution of 
SSOCT and PhOCT scans. Sensitivity on the order of angstroms has been reported [5] in PhOCT which corresponds with the findings of this project - average RMS wavelength jitter of in the range of $0.4-0.6 \mathrm{pm}$.

The VT-DBR performs well in OCT and has suitable stability of the wavelength sweep to obtain high-resolution images.

While long term wavelength wander does not impede OCT applications, many other forms of optical sensing are based on absolute wavelength. Moreover, these applications often require a source to sit idly for days or months without calibration.

An example of this is FBG sensing implemented to monitor railway traffic [15]. In such an application, many FBG sensors are placed on the railroad tracks. The sensors sit idly and measure the speed of a passing train, the number of axels, or other parameters. The sensors sit for an undetermined amount of time and are provide readings and information strictly based on their absolute wavelength, which shifts as a train passes overhead.

In such an application, the source that is sending light to the sensors must be completely reliable without drift. If the source drifts in wavelength, sensor readings may malfunction over prolonged periods of time.

This is the case in many fiber optic sensing applications: a source is required that does not wander over long periods of time. Therefore, we conclude that the VT-DBR in its present state can be employed for FBG and other sensing applications due to its small wander in absolute wavelength. This only applies to the 2 nd generation unit which exhibits very little wander. 
The external calibration algorithm developed in this project aids fiber sensing applications beyond OCT to correct for any wavelength wander. The only requirement is that a gas reference cell in the range of the laser sweep must be inserted into the system to monitor the laser sweep. This provides an absolute wavelength reference for the system. 


\section{FUTURE WORK}

There is much to learn and study about the VT-DBR laser system and its implementation in fiber sensing. This source will undoubtedly breach the fiber sensing market and provide benefits in a multitude of applications. Some projects that should be studied and undertaken with the laser are explored.

A standardized, modular calibration with an external wavelength reference should be finished. Ideally, such a routine should not be required because the VT-DBR will be absolutely stable in wavelength accuracy over long periods of time (i.e. months or years). As this work has discovered, however, the wavelength is not absolutely stable over time. Absolute wavelength calibration will be crucial to the fiber sensing market, where a sensor is often calibrated to a very specific wavelength, to sub-picometer accuracy. Even though a new unit shows significant improvements to wander, the problem still exists and must be compensated for.

This project utilized a gas reference cell as an absolute wavelength reference. For the purposes of this project, this was a long and tedious development process, resulting in a multi-stage calibration procedure which required several steps of human interaction and analysis. In order to streamline this process and work towards a successful external gas reference calibration, three main steps must be undertaken to simplify and automate the entire process.

First, the calibration algorithm must be refined and perfected. This algorithm may follow the same structure as used in this project or attempt a new approach. It will be necessary to employ a higher speed data acquisition system with a sampling rate of at 
least $400 \mathrm{MS} / \mathrm{s}$, if not $800 \mathrm{MS} / \mathrm{s}$. This will ensure that the data acquisition system can properly synchronize with the internal laser sample clock which operates at $400 \mathrm{MHz}$. Extensive work will need to be completed with regard to filtering out hold points from the sweep, thresholding the laser's electrical signals (data valid and start sweep), and aligning the start sweep signal with the data valid vector.

The second step towards successful gas cell calibration will be to calibrate a laser sweep with one gas reference cell and then calibrate the same sweep with a second, different reference cell. This will verify the robustness and accuracy of the calibration algorithm. This process will likely require some refinement and revision to the initial calibration routine in order for the calibrations from each cell to equal one another. Once this has been completed, any laser sweep may easily be referenced to absolute wavelengths and calibrated.

Finally, the third step for successful gas cell calibration is to integrate all components into the current hardware and software of the system. Future laser modules may incorporate a gas cell internally within the laser package. Furthermore, the gas cell calibration routine must be integrated with the current calibration routines and data processing algorithms to ensure that any data reflects an absolute wavelength reference to the user.

Another future work that will benefit the accuracy of the VT-DBR is multi-sweep averaging. Sweep averaging may be a method to resolve more precision out of the VTDBR sweeps. The Gaussian nature of jitter distribution observed in this project is the basis for multi-sweep averaging. 
This project discovered that the location of any single wavelength jitters around a mean value with a Gaussian distribution around this mean wavelength. Multi-sweep averaging proposes that by averaging this wavelength location over some number of sweeps, a more precise value may be obtained. This may ultimately allow for more precise sensor measurements or measurements with higher resolution.

Sweep averaging is made possible by the extremely high sweep repetition rates possible with the VT-DBR. Sweep rates up to $1 \mathrm{MHz}$ have been demonstrated. If an averaging length of 100 is chosen then a single wavelength location will be obtained for every 100 laser sweeps. Even with this method, a remarkably fast $10 \mathrm{kHz}$ effective sweep rate is still achieved.

Undertaking such a project will require a high speed data acquisition system (sampling rate of $2 \mathrm{MS} / \mathrm{s}$ at minimum) to ensure that the maximum sweep repetition rates can be acquired for analysis. It will also require high-speed analog-to-digital converters and digital signal processors that can acquire, process, average, and output relevant data at such high speeds.

Future work with regard to jitter will involve determining the root cause(s) within the laser system and finding ways to mitigate these effects. It is likely that the jitter observed jitter cannot be drastically reduced. In this case, post-processing such as multisweep averaging may assist in minimizing the effects of jitter for sensing applications. More immediate work should be undertaken to understand and correct for the effects of longer-term wander. Future work should investigate root causes more thoroughly of (1) temporal wavelength drift and (2) drift in amplitude or output power over time. Both of these effects are present in both the old and newer generation VT-DBR modules. 
Another future work will explore the VT-DBR in fiber sensing applications where long fiber lengths are implemented. The significant milestones of such a project will include aligning the data valid signal with a sweep signal that has propagated down tens or hundreds of kilometers of optical fiber. Employing an absolute wavelength reference such as a gas reference cell may aid this task greatly, but ideally, the laser system will operate without an external reference. 


\section{BIBLIOGRAPHY}

[1] M. -C. Amann and J. Buus, "Widely Tunable Laser Diodes," in Tunable Laser Diodes. Norwood: Artech House, Inc., 1998, ch. 7, pp. 159-163.

[2] Insight Photonic Solutions, "Akinetic All-Semiconductor Tunable Laser Source," User Manual 2013.

[3] M. A. Choma, M. V. Sarunic, C. Yang, and J. A. Izatt, "Sensitivity advantage of swept source and Fourier domain optical coherence tomography," Optics Express, vol. 11, no. 18, pp. 2183-2189, September 2003.

[4] M.P. Minneman, J. Ensher, M. Crawford, and D. Derickson, "All-semiconductor high-speed akinetic swept-source for OCT," in Proc. SPIE 8311, Optical Sensors and Biophotonics III, 831116 , 2011.

[5] J. Park, E. Carbajal, X. Chen, J. Oghalai, and B. Applegate, "Phase-sensitive optical coherence tomography in the middle ear using an akinetic swept laser source," Optics Letters, vol. 39, no. 21, pp. 6233-6236, 2014.

[6] M. Bonesi et al., "Akinetic all-semiconductor programmable swept-source at $1550 \mathrm{~nm}$ and $1310 \mathrm{~nm}$ with centimeters coherence length," Optics Express, vol. 22, no. 3, pp. 2632-2655, 2014.

[7] C. Karnutsch, STM 212. Class Lecture, Topic: "Optical Sensors." Sensor Systems and Technologies, Hochschule Karlsruhe, Technik und Wirtschaft, Karlsruhe, Germany, October 12, 2012.

[8] D. Talkington, "Characterization and Modeling of an O-Band $1310 \mathrm{~nm}$ Sampled- 
Grating Distributed Bragg Reflector (SG-DBR) Laser for Optical Coherence Tomography (OCT) Applications," California Polytechnic State University, San Luis Obispo, CA, MS Thesis 2013.

[9] K. Jeehyun et al., "Optical coherence tomography speckle reduction by a partially spatially coherent source," Journal of Biomedical Optics, 10(06)064034, 2005.

[10] Keysight Technologies, Understanding Jitter and Wander Measurements and Standards, 2nd ed., 2003.

[11] ThorLabs Instrumentation, "PDB100 Series Balanced Amplified Photodetectors," Datasheet 2007.

[12] Keysight Technologies, "Jitter Analysis Techniques Using Agilent Infiniium Oscilloscope," Appl. Note.

[13] W. C. Swann and S. L. Gilbert, "Accuracy limits for simple molecular absorption based wavelength references," National Institute of Standards and Technology, Boulder, CO, 2004.

[14] dBm Optics, "Gas Reference Cells," datasheet 2005.

[15] M. L. Filograno et al., "Real-Time Monitoring of Railway Traffic Using Fiber Bragg Grating Sensors," IEEE Sensors Journal, vol. 12, no. 1, pp. 85-92, January 2012.

[16] A. Ezbiri, S. E. Kanellopoulos, and V. A. Handerek, "High resolution instrumentation system for fibre-Bragg grating aerospace sensors," Optics Communications, vol.150, pp. 43-48, 1998.

[17] B. Culshaw and A. Kersey, "Fiber-Optic Sensing: A Historical Perspective," Journal of Lightwave Technology, vol. 26, no. 9, pp. 1064-1078, May 2008.

[18] A. Naumenko, P. Besnard, N. Loiko, G. Ughetto, and J. C. Bertreux, 
"Characteristics of a Semiconductor Laser Coupled with a Fiber Bragg Grating with Arbitrary Amount of Feedback," IEEE Journal of Quantum Electronics, vol. 39, no. 10, pp. 1216- 1228 , October 2003.

[19] C. D. Kissinger, "Fiber Optic Proximity Probe," 03327584, June 28, 1967.

[20] C. H. Lee, J. Lee, M. K. Kim, and K. T. Kim, "Characteristics of a Fiber Bragg Grating Temperature Sensor Using the Thermal Strain of an External Tube," Journal of the Korean Physical Society, vol. 59, no. 5, pp. 3188-3191, November 2011.

[21] D. Derickson et al., "SGDBR single-chip wavelength tunable lasers for swept source OCT," in SPIE 6847, Coherence Domain Optical Methods and Optical Coherence Tomography in Biomedicine XII, 68472P, 2008.

[22] Dennis Derickson, Fiber Optic Test and Measurement. Upper Saddle River, N. J. : Prentice Hall PTR, 1998.

[23] D. Talkington, D. Derickson, and J. Enscher, "O-band (1310 nm) Vernier-tuned distributed Bragg reflector (VT-DBR) device characterization for OCT," in SPIE 8934, Optical Coherence Tomography and Coherence Domain Optical Methods in Biomedicine XVIII, 89342X , 2014.

[24] Finisar, Appl. Note 2095, pp. 1-10.

[25] F. J. Duarte, Tunable Lasers Handbook. San Diego: Academic Press, 1995.

[26] H. G. Yu, Y. Wang, Q. Y. Xu, and C. Q. Xu, "Characteristics of Multimode Fiber Bragg Gratings and Their Influences on External-Cavity Semiconductor Lasers," Journal of Lightwave Technology, vol. 24, no. 4, pp. 1903-19112, April 2006.

[27] Insight Photonic Solutions. (2015, January) Akinetic All-Semiconductor 
Technology. [Online]. HYPERLINK "http://sweptlaser.com/akinetic-technology"

[28] I. Zamek and S. Zamek, "Definitions of Jitter Measurement and Relationships," in International Test Conference.

[29] J. R. Taylor, An Introduction to Error Analysis. Sausalito, CA: University Science Books, 1997.

[30] K. P. Zetie, S. F. Adams, and R. M. Tocknell, "How does a Mach-Zehnder interferometer work?," Physics Education, vol. 35, pp. 46-48, January 2000.

[31] K. R. Tamura, T. Komukai, and T. Morioka, "Modulation Requirements and Transmission Characteristics of Electrically Tunable Mode-Locked Lasers With Fiber Bragg Gratings," IEEE Photonics Technology Letters, vol. 14, no. 6, pp. 834-836, June 2002.

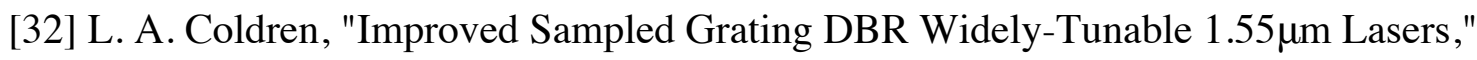
University of California, Santa Barbara, PhD Thesis 2000.

[33] M. Peach. (2014, October) Global Photonics Market Valued at More than \$150bn. [Online]. HYPERLINK "http://optics.org/news/5/9/24"

[34] M. Peach. (2014, October) Market for Fiber Optics Sensors to Hit \$4bn by 2017. [Online]. HYPERLINK "http://optics.org/news/4/6/17"

[35] M. Thonnard, "Confidence Intervals in Linear Regression," Technische Universiteit Eindhoven, Eindhoven, Germany, MS Thesis 2006.

[36] National Instruments, "Calibration Procedure NI 5105," 372249A-01 Datasheet 2007.

[37] National Instruments, "Getting Started Guide NI High-Speed Digitizers," 371133T Manual 2013. 
[38] National Instruments, "NI PXI/PCI-5105 Specifications," 374403G-01 Datasheet 2008.

[39] N. H. Sun et al., "Transmission and Reflection Characteristics of Fiber Bragg Gratings," in Proc. IEEE 2nd International Symposium on Next-Generation Electronics(ISNE), 2013, pp. 95-96.

[40] R. Kashyap, Fiber Bragg Gratings. San Diego, CA: Academic Press, 1999.

[41] SiTime, Appl. Note 10007 Rev 1.2.

[42] S. Niu, W. Rao, X. Zhang, and Y. Hu, "Phase Characteristics of Fiber Bragg Gratings and Fiber Fabry-Perot Cavities," in Proc. 2011 International Conference on Electronics and Optoelectronics, 2011, pp. 328-331.

[43] W. Beaty. (2004, January) Lasers: WTF is Coherent Light? [Online]. HYPERLINK "http://amasci.com/miscon/coherenc.html"

[44] Insight Photonic Solutions, "Akinetic All-Semiconductor Tunable Laser Source," Lafayette, Product Manual 2014.

[45] B. D. Cullity, Introduction to Magnetic Materials. Reading, MA: Addison-Wesley, 1972.

[46] S. M. Metev and V. P. Veiko, Kaser Assisted Microtechnology, 2nd ed., R. M. Osgood, Ed. Berlin, Germany: Springer-Verlag, 1998.

[47] Sakurambo. (2008, July) Fiber Bragg Gratings-en. [Online]. HYPERLINK "http://en.wikipedia.org/wiki/File:Fiber_Bragg_Grating-en.svg"

[48] (2015, February) Absorption Spectrum Graph. [Online]. HYPERLINK

"http://galleryhip.com/absorption-spectrum-graph.html"

[49] (2015, February) FBGS. [Online]. HYPERLINK 
"http://www.fbgs.com/technology/fbg-principle/"

[50] Paul F. Crowder, "Open loop control of SGDBR lasers ," Grant US6788719 B2, September 7, 2004. 